\title{
Update on therapeutic temperature management
}

\author{
Portoroz, Slovenia. 7-9 June 2012 \\ Edited by Gregor Broessner, Marlene Fischer, Gerrit Schubert, Bernhard Metzler and Erich Schmutzhard
}

Published: 7 June 2012

These abstracts are available online at http://ccforum.com/supplements/16/S2

\section{INTRODUCTION}

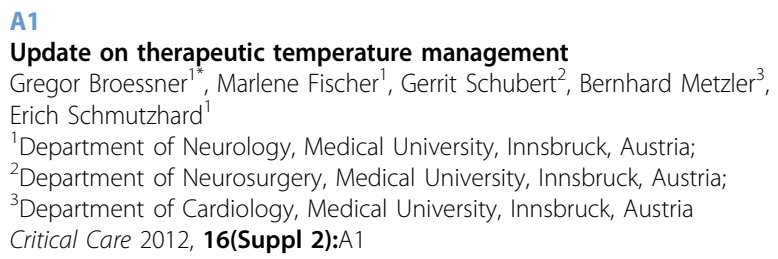

It is a pleasure to announce the 2nd Innsbruck Hypothermia Symposium. We are very happy that Critical Care has agreed to publish extended abstracts submitted by invited renowned scientists from all over the world; that is, Europe, the Americas, Asia. Neuroprotection - potentially achieved by targeted temperature management (that is, therapeutic hypothermia or prophylactic controlled normothermia) - is essential in emergency and acute care management of various severe neurologic and cardiologic diseases. Beyond neuroprotection - for this aim, therapeutic hypothermia has been established after resuscitation of patients with cardiac arrest due to a shockable arrhythmia and in neonatal asphyxic encephalopathy therapeutic hypothermia and prophylactic controlled normothermia have been published in single case reports, retrospective, open, but also in prospective randomised controlled trials in many other emergency disciplines in which both neuroprotection and protection of other organs and tissues are the target of our therapeutic endeavours. The Medical University Innsbruck, Austria, is happy to organise this conference on temperature management, therapeutic hypothermia and prophylactic normothermia respectively, to be held in Portoroz, Slovenia. In accordance with the first Meeting on Hypothermia, which was held in Miami, Florida, USA (CHilling At the Beach), we are proud to suggest the acronym $\mathrm{CHAB}$ standing for take Care for Heart And Brain, characterising the major target organs of therapeutic and, possibly also, prophylactic temperature management. Again, we have been able to gather most renowned scientists, neurointensivists and intensivists, emergency physicians, cardiologists and other specialists to cover the entire scientific and clinical spectrum of emergency temperature management, technical aspects of cooling and management of potential complications including shivering, but also temperature management in neurology, neurosurgery, intensive care medicine, in the operation theatre, cardiology, infectious diseases, and so forth. Beyond that we cross borders and discuss hypothermia and intracranial pressure, pharmacodynamics in hypothermic patients and the influence of hypothermia onto pharmacokinetics/pharmacodynamics, hypothermia in refractory status epilepticus or heat stroke, hypothermia and advanced neuromonitoring, hypothermia and nutrition, shivering and the critical issue of rewarming, amongst other topics.

The aim of this symposium is to enhance the knowledge on temperature management, increase the readiness and stimulate the preparedness to institute therapeutic hypothermia and/or prophylactic controlled normothermia, respectively, in patients in need of tissue and organ protection, uncontrolled body temperatures possibly adding - per se - to neuronal damage. Knowing the medical literature and knowing the issue of potentially life-threatening side effects and complications incurred by this invasive therapeutic manoeuvre, it is the foremost aim of this symposium and this supplementary issue of Critical Care to discuss all these aspects of targeted temperature management in emergency, critical care and, in particular, neurocritical patients and conditions. For this reason the organisers have agreed that the discussion of these various issues, being so important for general critical care, neurocritical care and emergency medicine, must be distributed as widely as possible, making it available to critical care and neurocritical care specialists all over the world. Therefore we are extremely grateful to the Editors of Critical Care for providing a forum for all of the extended abstracts of all invited speakers, covering the entire field of adult emergency and critical care medicine. We do hope and we are convinced that this supplementary issue will be a source of inspiration and knowledge, hopefully becoming a work of reference for intensivists, neurologists, neurointensivists, cardiologists and all emergency physicians alike. It is the aim of the organisers to establish a series of such symposia within the next years in order to keep up with all the developments in this field and to maintain the highest possible level of knowledge of targeted temperature management in the community of emergency and intensive care physicians.

\section{EMERGENCY TEMPERATURE MANAGEMENT}

\section{A2}

Therapeutic hypothermia: the rationale

Erich Schmutzhard*, Marlene Fischer, Anelia Dietmann, Gregor Brössner Department of Neurology, Neurocritical Care Unit, Medical University Innsbruck, Austria

Critical Care 2012, 16(Suppl 2):A2

For almost a century, therapeutic hypothermia - or as it was termed in the early days: hibernation - has been discussed as a potential neuroprotective measure, in particular in patients suffering from severe intracranial disease leading to impairment of consciousness, associated with fever [1-3].

In a wide range of diseases, secondary damage to the brain or other organs follows the initial impact and may be responsible for aggravation of disease condition or clinical state, in particular neurological morbidity and/ or mortality [4-11]. Therapeutic hypothermia, recently renamed targeted temperature management, including prophylactic normothermia, has been used to improve this secondary impact onto brain and other organ tissue. This holds true, in particular, for neurological and neurosurgical intensive care patients since secondary brain and nervous tissue injury may preclude a potentially benign course of disease. The mechanisms of action of hypothermia are complex, not yet fully understood. Therapeutic hypothermia/targeted temperature management aims to attenuate a cascade of secondary injury mechanisms, which is started immediately 
after the initial event (primary injury) and may last for hours and even days $[4,6,12]$. The majority of research has focused, so far, on these secondary injury processes being destructive to brain and nervous tissue. It may be expected that any such protective effect can be replicated in other organs and tissues during therapeutic hypothermia/targeted temperature management. A wide range of side effects may negate and counteract its positive initial effect; this implies side effects of hypothermia per se and side effects of rewarming or inconstant maintenance of temperature levels [13-17].

This abstract limits itself to potential pathophysiological mechanisms of actions, the risks of any such mechanism and side effects derived from them $[4,5,10,12,16-18]$.

The protective effect of hypothermia may be explained by several pathways. A decreased metabolism with less oxygen and energy consumption and carbon dioxide production may prevent secondary injury when oxygen supply is interrupted or, at least, impaired. However, it needs to be stressed that the reduction in metabolic rate, as seen in hypothermia, requires adjustment in ventilator setup, insulin infusion rate, correct interpretation of electrolytes, in particular low phosphate, magnesium and potassium levels. Of particular interest are the rebound phenomena during rewarming or when, involuntarily, the temperature cannot be maintained at the targeted low level.

Following ischemia, hypoxia or direct trauma apoptotic processes may be initiated in brain tissue and neuronal cells may even become necrotic. In these earliest stages these pathways may be blocked by hypothermia. However, little is known about the time frame and best window of opportunity to use therapeutic hypothermia to prevent initiation of apoptotic/necrotic processes.

Any type of neuronal injury may provoke the neuro-excitatory cascade, starting off with excessive calcium influx, glutamate receptor activation, neuronal hyperexcitability, eventually leading to cell death even after reperfusion and normalization of glutamate levels. It has been suggested that therapeutic hypothermia may reduce cellular/neuronal damage following this neuro-excitatory cascade.

It has been accepted that the release of free radicals may be deleterious to both neuronal cells and the brain's defense mechanisms alike. Whether the direct impact or the ischemia reperfusion injury is overwhelmingly responsible for the release/increase of free radicals oxidizing and damaging neuronal cell components is both still a matter of discussion and of limited interest when therapeutic hypothermia comes into play.

Hyperexcitability, cellular hyperactivity, mitochondrial dysfunction, ionpump failure and reduction in cellular membrane integrity may lead to intracellular and, consequently, also intercellular/extracellular acidosis.

Early initiation of hypothermia may improve this full spectrum of cellular failure, improve brain glucose and energy metabolism and reduce lactate accumulation; with this, intracellular and intercellular acidosis will improve and eventually metabolic recovery be enhanced [4,19-21].

Any type of brain injury is capable of disrupting the blood brain barrier leading to enhanced vascular permeability, brain edema, vascular permeability and perivascular hemorrhage. Brain edema, both after ischemia/hypoxia and traumatic injury peaks after 24 to 72 hours (sometimes reaching its highest peak even after this period of time) - thus opening widely the therapeutic window - allowing for therapeutic hypothermia to reduce brain edema via stabilizing the disrupted bloodbrain barrier and vascular permeability. After brain injury proinflammatory mediators are released, leucocytes cross the - already impaired - bloodbrain barrier leading to an accumulation of inflammatory cells in the brain. This inflammatory response starts within 1 hour after injury and may persist for up to 5 days, a fact which also suggests a widely open therapeutic window for intervention. Hypothermia has been shown to reduce ischemia induced inflammatory and immune reactions [4,19-22]. In healthy persons, brain temperature is around 0.5 to $1^{\circ} \mathrm{C}$ higher than core body temperature. In any type of brain injury, in particular, in patients with fever or hyperpyrexia respectively, injured areas may be definitely hotter (up to $2^{\circ} \mathrm{C}$ post injury), most probably due to transitory cellular hyperactivity. Local brain edema might lead to cerebral thermopooling adding to hyperthermia-related neuronal cellular injury [4,16,18-21].

Cooling below $35^{\circ} \mathrm{C}$ has been shown to affect coagulation, it depends on the initial type of brain injury whether a procoagulatory effect or an anticoagulatory effect is believed to be neuroprotective in an individual case. Targeted temperature management may influence the secretion of vasoconstricting substances (for example, endothelin) or vasodilating substances (for example, prostaglandins). Their balance is essential to maintain homeostasis. Ischemic or traumatic conditions may increase vasoconstricting substances thus leading to reduced cerebral blood flow. Whether hypothermia is capable of regulating/improving cerebral perfusion is still a matter of investigation, pending the influence of cerebral autoregulation and the quantity of secreted vasoactive mediators in brain-injured patients with cerebral ischemia or any other type of injury [10].

Whether epileptic activity, in particular, subtle nonconvulsive status epilepticus, accepted to indicate severe brain damage, can be positively influenced by therapeutic hypothermia still needs further research. However, it is accepted that a subtle nonconvulsive status epilepticus occurring in the acute phase of brain injury is - per se - adding to neuronal destruction $[10,16]$.

While many pathophysiological processes and cascades may be influenced by targeted temperature management/therapeutic hypothermia and/or even prevention of fever through prophylactic normothermia, it is unclear whether in all types of severely brain-injured patients (for whatever reason) the benefits of this therapeutic hypothermia always outweigh its risks. It is now fully accepted and of a high level of evidenced medicine that in cerebral hypoxia (in a patient with cardiac arrest due to a shockable arrhythmia) as well as asphyxial encephalopathy a 24-hour therapeutic hypothermia (33 to $34^{\circ} \mathrm{C}$ ), irrespective of the type of cooling, improves neurological outcome; that is, morbidity but also mortality $[7,10]$. Whether therapeutic hypothermia/ targeted temperature management or prophylactic normothermia may improve outcome in other diseases, as discussed in this meeting, is still not clear. It needs to be stressed that even such seemingly similar diseases as global hypoxia (in cardiac arrest due to a shockable arrhythmia), asphyxial encephalopathy and ischemic stroke have so few pathophysiologic cascades in common. Therefore, they may not be treated all alike, in particular, with respect to type, duration, speed and depth of hypothermia as well as rewarming management [23]. It has already been demonstrated that in hypoxic encephalopathy hypothermia for 24 hours may be sufficient. However, disease entities such as ischemic stroke, hemorrhagic stroke with formation of peri-hematomal edema, traumatic brain injuries with prolonged secondary insult or the wide range of neuronal injuries after subarachnoid hemorrhage may present even more complex pathophysiologic mechanisms. Moreover, different pathologies such as encephalitis and bacterial meningitis or even spinal cord injury may all require a targeted and personalized approach to this adjunctive therapy. In some cases, prolonged hypothermia may be equally necessary as in other cases mild hypothermia or even only prophylactic normothermia may suffice.

It may be stated beyond doubt that the neuroprotective effect of moderate hypothermia $\left(33\right.$ to $34^{\circ} \mathrm{C}$ ) has been shown in cerebral hypoxia and asphyxial encephalopathy. However, different neurocritical care disease entities as discussed above have different mechanisms of primary insults as well as the mechanisms and cascades of secondary brain injury and therefore require a different therapeutic approach in respect of temperature management.

Any type of therapeutic measure, still being the subject of research, must never harm the patient. Hypothermia-induced neurological signs and symptoms must never be misinterpreted and as a matter of course the diagnosis of brain death can never be confirmed under hypothermic conditions [24].

\section{References}

1. Kellock B: The Fibreman, the Life Story of Dr Denis Burkitt Lion Publishers; Oxford Oxfordshire OX2 7DH, UK, 1st 1985, p8.

2. Zdravev P: Treatment of cerebral hernia following surgery of an otogenous brain abscess. Ann Otolaryngol 1951, 68:201-205.

3. Delgado BJ: Otogenous cerebral abscess treated by surgical intervention and hibernation. Acta Otorinolaryngol Iber Am 1956, 7:212-220.

4. Andrews PJ, Sinclair HL, Battison CG, et al: European society of intensive care medicine study of therapeutic hypothermia $\left(32^{\circ}-35^{\circ} \mathrm{C}\right)$ for intracranial pressure reduction after traumatic brain injury (the Eurotherm 3235 Trial). Trials 2011, 12:12-18.

5. Benz-Woerner J, Delodder F, Benz R, et al: Body temperature regulation and outcome after cardiac arrest and therapeutic hypothermia. Resuscitation 2012, 83:338-342.

6. Childs C: Human brain temperature: regulation, measurement and relationship with cerebral trauma: part 1. Br J Neurosurg 2008, 22:486-496.

7. Delhaye C, Mahmoudi M, Waksman R: Hypothermia therapy neurological and cardiac benefits. J Am Coll Cardiol 2012, 59:197-210. 
8. Dietrich WD, Cappuccino A, Cappuccino H: Systemic hypothermia for the treatment of acute cervical spinal cord injury in sports. Curr Sports Med Rep 2011, 10:50-54

9. Dietrich WD: Therapeutic hypothermia for acute severe spinal cord injury: ready to start large clinical trials? Crit Care Med 2012, 40:691-692.

10. Moore EM, Nichol AD, Bernard SA, Bellomo R: Therapeutic hypothermia: benefits, mechanisms and potential clinical applications in neurological, cardiac and kidney injury. Injury 2011, 42:843-854.

11. Rivera-Lara L, Zhang J, Muehlschlegel S: Therapeutic hypothermia for acute neurological injuries. Neurotherapeutics 2012, 9:73-86.

12. Polderman $\mathrm{KH}$, Herold I: Therapeutic hypothermia and controlled normothermia in the intensive care unit: practical considerations, side effects, and cooling methods. Crit Care Med 2009, 37:1101-1120.

13. Broessner $G$, Beer $R$, Helbok $R$, et al: Prophylactic, endovascularly based, long-term normothermia in ICU patients with severe cerebrovascular disease: bicenter prospective, randomized trial. Stroke 2009, 40:657-665.

14. Cueni-Villoz N, Devigili A, Delodder F, et al: Increased blood glucose variability during therapeutic hypothermia and outcome after cardiac arrest. Crit Care Med 2011, 39:2225-2231.

15. Fischer $M$, Dietmann $A$, Lackner $P$, et al: Endovascular cooling and endothelial activation in hemorrhagic stroke patients. Neurocrit Care 2011 in press.

16. Polderman KH: Mechanisms of action, physiological effects, and complications of hypothermia. Crit Care Med 2009, 38:186-202.

17. Polderman KH: Hypothermia, immune suppression and SDD; can we have our cake and eat it? Crit Care 2011, 15:144.

18. Sacho RH, Childs C: The significance of altered temperature after traumatic brain injury: an analysis of investigations in experimental and human studies: part 2. Br J Neurosurg 2008, 22:497-507.

19. Broessner $G$, Lackner $P$, Fischer $M$, et al: Influence of prophylactic, endovascularly based normothermia on inflammation in patients with severe cerebrovascular disease: a prospective, randomized trial. Stroke 2010, 41:2969-2972.

20. Fischer $M$, Lackner $P$, Beer $R$, et al: Keep the brain cool - endovascular cooling in patients with severe traumatic brain injury: a case series study. Neurosurgery 2011, 68:867-873.

21. Childs C, Wieloch T, Lecky F, et al: Report of a consensus meeting on human brain temperature after severe traumatic brain injury: its measurement and management during pyrexia. Front Neurol 2010, 1:146

22. Todd MM, Hindman BJ, Clarke WR, et al: Perioperative fever and outcome in surgical patients with aneurysmal subarachnoid hemorrhage. Neurosurgery 2008, 64:897-908.

23. Perman SM, Kirkpatrick JN, Reitsma AM, et al: Timing of neuroprognostication in postcardiac arrest therapeutic hypothermia. Crit Care Med 2012, 40:719-724

24. Webb AC, Samuels OB: Reversible brain death after cardiopulmonary arrest and induced hypothermia. Crit Care Med 2011, 39:1538-1542.

\section{A3}

\section{Prehospital hypothermia}

Hans-Jörg Busch" ${ }^{1 *}$, Katrin Fink ${ }^{2}$

${ }^{1}$ Emergency Department, University Hospital Freiburg, Germany; ${ }^{2}$ Department of Cardiology and Angiology, University Hospital Freiburg, Germany

Critical Care 2012, 16(Suppl 2):A3

Mild hypothermia is widely used in the treatment of successfully resuscitated patients after cardiac arrest [1]. Previous experimental and clinical studies have demonstrated beneficial effects of cooling after cardiac arrest. Two clinical landmark studies in 2002 demonstrated the use of therapeutic hypothermia after cardiac arrest due to ventricular fibrillation decreases mortality and improves neurological outcome $[2,3]$. This led the International Liaison Committee on Resuscitation and the American Heart Association to recommend the use of therapeutic hypothermia after cardiac arrest as soon as possible after the return of spontaneous circulation (ROSC) [4].

Despite major progress in intensive care medicine in the last decades, mortality rates after cardiac arrest remain unacceptably high [2,3]. The high mortality rates after cardiac arrest can be attributed to a unique pathophysiological process $[1,5,6]$. The entity of the pathophysiological changes after ROSC - for example, activation of the inflammatory system can be summarized as the post-cardiac arrest syndrome [1,5-7].
Hypoxic encephalopathy, which is often a result of the initial hypoxic phase and/or the post-cardiac arrest syndrome, is one of the main causes for mortality, disability and a need for permanent care in patients after cardiac arrest [1].

Pathophysiologically, the resuscitation period could be divided into different time periods. After cessation of circulation, ischemia of different tissues leads to necrotic cell death (hypoxia-induced cellular dysfunction) $[7,8]$. Reperfusion injury then follows after an imprecise period of time once oxygenated blood is returned to the ischemic tissues with the beginning of mechanical resuscitation (reperfusion-induced cell death) $[7,8]$. From experimental and clinical studies, it is clear that the tissue damage due to reperfusion occurs over several hours to days in the postresuscitation phase $[1,7,8]$.

Several experimental studies have emphasized induction of therapeutic hypothermia as soon as possible after ROSC or during cardiopulmonary resuscitation [7-10]. These studies in the different animal models demonstrate a beneficial effect, including attenuation of the cerebral injury after prolonged ischemia due to earlier cooling [7-10]. Recent experimental data in different animal models of cardiac arrest, stroke and myocardial infarction suggest that warm reperfusion under normal or hyperthermic conditions could increase the deleterious effects of the reperfusion. For the effective prevention and treatment of the reperfusion injury, reperfusion should occur in temperature-controlled or cooled tissues.

Nevertheless, prehospital induction of therapeutic hypothermia is still under discussion; consistent protocols are not present and human data are rare. In a retrospective clinical study, early achievement of the target temperature appeared to reduce hypoxic brain injury and favor a good neurologic outcome after successful resuscitation [11].

On the other hand, a small retrospective, observational investigation found a faster decline in body temperature to the target temperature is linked to a less favorable neurologic outcome in comatose patients after cardiac arrest treated with therapeutic hypothermia [12]. However, this may simply indicate a severe ischemic damage with consecutive impaired thermoregulation [12].

In the PRINCE study, feasibility of preclinical transnasal cooling with evaporated perfluorcarbon that primarily leads to a prior selective cooling of the cerebrum was analyzed. In a subgroup of patients, intra-arrest hypothermia via evaporated perfluorcarbon was beneficial $[13,14]$. Several other studies show also safety and feasibility of prehospital hypothermia $[15,16]$. In summary, prehospital treatment of patients with a cardiac cause of the arrest may increase the rate of favorable outcome at hospital discharge. Further larger clinical investigations are needed to evaluate the effects of prehospital cooling in cardiac arrest patients $[7,8]$. In a small survey of emergency physicians in Germany, only a minority of patients is frequently treated with hypothermia before hospital admission after successful resuscitation $[7,8]$.

However, taking the pathophysiological processes into consideration induction of therapeutic hypothermia should not be limited to the ICUs but should also be able in the field or in the emergency department. Different methods are available to achieve and maintain the target temperature in the prehospital setting $[7,8]$.

References

1. Nolan JP, Neumar RW, et al: Post-cardiac arrest syndrome: epidemiology, pathophysiology, treatment, and prognostication. Resuscitation 2008, 79:350.

2. Hypothermia After Cardiac Arrest Study Group: Mild therapeutic hypothermia to improve the neurologic outcome after cardiac arrest. N Engl J Med 2002, 346:1756.

3. Bernard SA, Gray TW, Buist MD, et al: Treatment of comatose survivors of out-of-hospital cardiac arrest with induced hypothermia. N Engl J Med 2002, 346:557.

4. ECC Committee, Subcommittees and Task Forces of the American Heart Association: American Heart Association guidelines for cardiopulmonary resuscitation and emergency cardiovascular care. Circulation 2005, 112(24 Suppl IV):1-203.

5. Negovsky VA: The second step in resuscitation: the treatment of the 'post-resuscitation disease'. Resuscitation 1972, 1:1-7.

6. Fink $K$, Feldbrügge $L$, Schwarz $M$, et al: Circulating annexin V positive microparticles in patients after successful cardiopulmonary resuscitation. Crit Care 2011, 15:R251.

7. Taccone FS, Donadello $\mathrm{K}$, Beumier $\mathrm{M}$, et al: When, where and how to initiate hypothermia after adult cardiac arrest. Minerva Anestesiol 2011 77:927-933. 
8. Lampe JW, Becker LB: State of the art in therapeutic hypothermia. Annu Rev Med 2011, 62:79-93.

9. Boddicker KA, Zhang Y, Zimmerman MB, et al: Circulation 2005, 111:3195-3201.

10. Zhao D, Abella BS, Beiser DG, et al: Resuscitation 2008, 77:242-249.

11. Wolff B, Machill K, Schumacher D, et al: Early achievement of mild therapeutic hypothermia and the neurologic outcome after cardiac arrest. Int J Cardiol 2009, 133:223-228.

12. Haugk M, Testori $C$, Sterz F, et al: Relationship between time to target temperature and outcome in patients treated with therapeutic hypothermia after cardiac arrest. Crit Care 2011 in press.

13. Castrén $M$, Nordberg $P$, Svensson $L$, et al: Intra-arrest transnasal evaporative cooling: a randomized, prehospital, multicenter study (PRINCE: Pre-ROSC IntraNasal Cooling Effectiveness). Circulation 2010, 122:729-736

14. Busch HJ, Eichwede F, Födisch M, et al: Safety and feasibility of nasopharyngeal evaporative cooling in the emergency department setting in survivors of cardiac arrest. Resuscitation 2010, 81:943-949.

15. Bernard SA, Smith K, Cameron P, et al: Rapid Infusion of Cold Hartmanns (RICH) Investigators: Induction of prehospital therapeutic hypothermia after resuscitation from nonventricular fibrillation cardiac arrest. Crit Care Med 2012, 40:747-753.

16. Busch HJ, Brendle V, Bode C, Koberne F, Schwab T: Prehospital hypothermia after cardiac arrest a survey the in emergency physician based ambulance system in Baden-Wuerttemberg, Germany. Notfall Rettungsmed 2011, 11:1474-1480.

A4

Standard operating procedures: therapeutic hypothermia in CPR and post-resuscitation care

Markus J Foedisch, Andreas Viehoefer

Department of Anesthesia and Intensive Care Medicine, Evagelische Kliniken Bonn, Germany

Critical Care 2012, 16(Suppl 2):A4

After two randomised studies published in $2002[1,2]$ mild therapeutic hypothermia treatment was internationally recommended as early and efficious treatment for comatose survivors after cardiac arrest (CA) not only with ventricular fibrillation, but also for patients suffering from CA presenting with other initial rhythms (asystole, PEA) and different underlying causes. Therapeutic hypothermia has been shown in these investigations to improve not only survival significantly after CA but especially the neurologic outcome after different courses of cooling treatment. Nevertheless the in-hospital mortality of those patients remained high.

While several prehospital or pre-CPR factors contributing to the patients outcome are well known and implemented in the BLS and ACLS guidelines, only little is known about the kind and impact of in-hospital contributing factors worsening the chance of surviving the event with good neurological function. After return of spontaneous circulation, major cardiovascular and haemodynamic disorders are widely common and associated with a high rate of deaths within the first 24 hours after CPR. Sufficient post-resuscitation therapy has to include optimal treatment strategies of the cardiovascular and metabolic system, adaequate ventilation support and strategies of neuroprotection [3]. In patients surviving with a favourable outcome, haemodynamic and respiratory disorders tend to normalise within the first 24 hours after ROSC.

Several factors of hospital care are obviously important for survival of postCA patients. Observational investigations done in Norway and Sweden detected severe differences in outcome of patients admitted to hospital with ROSC after out-of-hospital CA presenting survival rates between 33 to $56 \%$ and 14 to $42 \%$ respectively [4-6]. There were no significant differences in the prehospital management of those patients, but in-hospital factors like blood glucose levels, seizures, body temperature and laboratory changes could be related to outcome. A similar cohort study using a multicentre clinical ICU registry in the United States enrolled 4,674 patients from 39 hospitals covering a 4-year hiatus showed the same interhospital variability in survival with an unadjusted mortality ranging from 41 to $81 \%$. Those patients treated in centres with higher case volumes were significantly less likely to die in-hospital after ROSC independent of the location of the CA. As it was not possible to differentiate the effect of specific therapies and interventions on survival in the post-CA period, the results underlined the need for additional research to define optimal postcardiac treatment strategies. The data underlined not only the volumeoutcome relationship but also the necessity of implementing standardised guidelines for optimal post-CA care in specialised centres.

Based on this evidence a prospective observational study was performed in patients admitted to hospital after regaining ROSC and treated using a standardised treatment protocol including instant onset of therapeutic hypothermia, early reperfusion treatment with $\mathrm{PCl}$, and protocol-based earlygoal-directed therapy to restore adaequate arterial blood flow in the reperfusion period [7]. The observational group from the interventional period was compared with controls from an earlier period in the same hospital. There were not only major differences in survival but also in the quality of neurologic outcome. After implementation of the standardised treatment protocol, survival improved from $31 \%$ to $56 \%$ in the interventional period, $56 \%$ of the patients showed a favourable neurologic outcome ( $26 \%$ in the control period) at hospital discharge and were still alive after 1 year. With no changes in the algorithm of prehospital care in the years of the investigation, post-resuscitation care appeared to have a major effect on improving not only survival but also the neurologic outcome after successful CPR.

Despite the fact that the level of evidence for many of the treatment strategies with the exception of therapeutic hypothermia in postresuscitation care is weak, the quality of care after admission to the ICU or ED seems to be a somewhat missing link in the chain of survival. The post-resuscitation phase is associated with a sepsis-like syndrome [8] of unknown time course causing or even intensifying global ischaemic brain damage and dysfunctional heart disease. Treatment of these disorders is the main challenge after ROSC, but implementation of such strategies is often slow and in a heterogeneous manner causing a widely variable state of post-resuscitation care.

Many factors (Table 1) may contribute to this phenomenon and show the complexity of treating patients after ROSC. This underlines the necessity of using protocol-driven care in those patients to help physicians and nurses to raise the level for the number of patients receiving standard therapy. It is obvious that such protocols have to be adapted to local hospital specialities and logistic factors.

In our hospital an early algorithm for therapeutic hypothermia based on the standards used during the HACA trial [1] was designed and implemented immediately after enrolling patients for that European multicentre study in 2001. All patients being successfully resuscitated after CA independent from localisation, initial rhythm and type of the event were treated by therapeutic hypothermia and enrolled in our own database (CoolBrain Registry Bonn) including EMS data, course and technique of cooling and following temperature management, neurologic outcome at discharge and in a 1-year follow-up. Shortly after implementing the cooling protocol a special algorithm for general postresuscitation care including therapeutic hypothermia and focusing on an early goal-directed approach to cardiac function, normoventilation, seizure treatment and strict avoidance of high blood glucose levels was designed and enabled physicians and nurses how to monitor and treat those patients. Baseline data of heart and brain function using invasive cardiac output monitoring and brain damage markers were included in the database as well. Both protocols and order sets are actualised to new guidelines and therapeutic standards based on actual science on a regular basis.

\section{Table 1(Abstract A4) Inhospital factors influencing outcome of CA patients}

Lack of implementation of therapeutic hypothermia and temperature management

Missing standard operating procedures/protocols for post-resuscitation care

Time lapse from ROSC to start of interventional phase

Treated case volumes of CA patients

Training and experience of personnel

Inadequate post-arrest treatment decisions 
Unfortunately there is only limited access to outcome data of our patients treated before implementation of these SOP protocols, so the data from the CoolBrain Registry are only observational and cannot be compared with a control group before using protocol-driven therapeutic standards. Neurologic outcome was not recorded before implementing therapeutic hypothermia as standard care in 2001; survival rates after OHCA were recorded between 1990 and 2000 as lower than $20 \%$.

Data enrolled from March 2001 to December $2011(n=276)$ presented a general survival rate of $51 \%$ independent of origin, initial rhythm and localisation of the cardiac arrest. Thirty-nine per cent of those patients showed a favourable neurologic outcome (CPC 0.5), 12\% severe neurologic disability. In the group of survivors, $69.5 \%$ of patients with ventricular fibrillation as the initial rhythm showed an excellent neurologic performance (CPC 1) and 35\% of patients presenting with asystole/PEA as well at hospital discharge.

Despite missing a control group before implementing therapeutic hypothermia and standardised treatment protocols for post-resuscitation care, these data show the effect of protocol-based treatment especially on neurologic outcome of those patients. This underlines that more attention has to be focused on optimising and standardising post-resuscitation care to improve survival and neurologic outcome. Treatment of patients with CA does not end with ROSC; despite the fact that there is only weak scientific level of evidence for some singular strategies of post-CA treatment, the combination of an aggressive multifactorial therapeutic approach including temperature management significantly improves outcome. Therefore further clinical trials of other post-resuscitation therapies seem to be essential.

\section{References}

1. Hypothermia After Cardiac Arrest Study Group: Mild therapeutic hypothermia to improve the neurologic outcome after cardiac arrest. N Engl J Med 2002, 346:549-563.

2. Bernard SA, Gray TW, Buist MD: Treatment of comatose survivors of outof-hospital cardiac arrest with induced hypothermia. N Engl J Med 2002, 346:557-563.

3. Gaieski DF, Band RA, Abella BS, et al: Early goal directed hemodynamic optimization combined with hypothermia in comatose survivors of outof-hospital cardiac arrest. Resuscitation 2009, 80:418-424.

4. Langhelle A, Tyvold SS, Lexow K, et al: In-hospial factors associated with improved outcome after out-of-hospital cardiac arrest. A comparison between for regions in Norway. Resuscitation 2003, 56:247-263.

5. Herlitz J, Engdahl J, Svensson L, et al: Major differences in 1 month survival between hospitals in Sweden among initial survivors of out-ofhospital cardiac arrest. Resuscitation 2006, 70:404-409.

6. Carr BG, Kahn JM, Merchant RM, et al: Inter-hospital variability in postcardiac arrest mortality. Resuscitation 2009, 80:30-34

7. Sunde K, Pytte $M$, Jacobsen $D$, et al: Implementation of a standardised treatment protocol for post resuscitation care after out-of-hospital cardiac arrest. Resuscitation 2007, 73:29-39.

8. Adrie C, Adip-Conquy M, Laurent I, et al: Successful cardiopulmonary resuscitation after cardiac arrest as a 'sepsis'-like' syndrome. Circulation 2002, 106:562-568.

\section{A5}

\section{In-hospital hypothermia}

Christian Storm

Department of Nephrology and Medical Intensive Care Medicine, Charité Universitätsmedizin Berlin, Campus Virchow-Klinikum, Berlin, Germany Critical Care 2012, 16(Suppl 2):A5

Introduction: Mild therapeutic hypothermia after cardiac arrest has become standard in post-resuscitation care in many hospitals as it is recommended by current guidelines. The last update of guidelines by the European Resuscitation Council on post-cardiac arrest treatment in 2010 recommends hypothermia for every patient after cardiac arrest who remains unconscious after cardiac arrest [1]. In addition to milestone trials [2-4], current published retrospective data from the large Finnish registry showed in a large group of patients a significant reduction of hospital mortality of survivors of out-ofhospital cardiac arrest after implementation of hypothermia [5].

The mild therapeutic hypothermia procedure after cardiac arrest can be divided into three phases: introduction, maintenance and rewarming. The cooling techniques and devices to induce cooling of the cardiac arrest survivor can be separated into three main groups: conventional cooling (no device), non-invasive (surface) systems, and invasive (intravascular) systems (Table 1).

Cooling techniques: Conventional cooling methods: The easiest way to induce hypothermia after cardiac arrest is by using cold saline (for example, $0.9 \% \mathrm{NaCl}$ solution), crushed ice or ice bags. Kim and colleagues reported the safety and efficacy of the administration of up to 2 litres of $4^{\circ} \mathrm{C}$ cold saline to the patient after hospital admission [6]. Others published data using $30 \mathrm{ml} / \mathrm{kg}$ body weight of saline $0.9 \% \mathrm{NaCl}$ or Ringer's lactate combined sometimes with ice bags, which led to an acceptable reduction of the temperature [7-10]. Furthermore cold saline as well as other methods like cooling caps and helmets have been evaluated for induction mainly in the preclinical setting $[4,11,12]$. Kliegel and colleagues pointed out that cold infusion alone is effective for induction but not for tight maintenance of the target temperature [13]. However, in at least one trial the combination of cold saline and ice packs was proven to be effective even to maintain temperature [7]. Focusing on the induction in the in-hospital setting, most authors rank cold saline and crushed ice more as effective adjuvant methods to be combined with a computer-controlled cooling device [10]. The big advantage of cold saline is its availability at almost every place in the hospital if provided and the low costs. Following the data available concerning different amounts of saline administered to the patient, a median amount of 1 to 2 litres of saline seems safe after cardiac arrest. To maintain target temperature with cold saline and ice bags seems to require a high binding of personnel method without a very precise influence on the central body temperature.

Surface cooling methods: Surface, non-invasive devices have to be distinguished from intravascular, invasive devices. The range of available computer-controlled surface devices with automatic temperature feedback stretches from cooling blankets to be placed around the patient (Blanketrol III, Cincinnati Sub-Zero; CritiCool, Medical ThermoRegulation Expertise) to adhesive cooling pads (Arctic Sun, Bard). Heard and colleagues compared the adhesive Arctic Sun surface-cooling system with normal cooling blankets combined with ice bags. Although the reached target temperature within 4 hours was not significantly different between the groups, the Arctic Sun system cooled more rapidly down to the target temperature [14]. A current investigation from Norway compared the Arctic Sun surface (C.R. Bard) system $(n=92)$ with the invasive intravascular Coolgard (Alsius) system ( $n=$ 75) in cardiac arrest survivors. The authors concluded no significant differences concerning neurological outcome and survival at discharge. A limitation for interpretation of the device efficacy (cooling rate/hour) is the additional induction of cooling with cold saline and ice bags already in the emergency room [15]. A published case report described a severe skin peeling during hypothermia with the Arctic Sun system without a known history of skin problems or steroid therapy but with end-stage renal disease and coronary artery disease. This is the first severe adverse skin event towards the hydrogel pads known and the authors conclude that these skin lesions are very unusual as to be caused by the adhesive pads because exfoliative dermatitis is a rare syndrome and is often drug induced [16]. Thus adverse skin reactions should not normally be expected using this method of cooling.

Another surface feedback system using blankets is the CritiCool Pro system by Medical ThermoRegulation Expertise (MTRE, Israel). The patient is wrapped into the body-shaped heat exchange garment resulting in a median cooling rate of $0.7 \pm 0.37^{\circ} \mathrm{C} /$ hour in a study by Laish-Farkash and colleagues [17]. The Cincinnati Sub-Zero system has been compared with the ArcticSun2000 (C.R. Bard) system by Mayer and colleagues for fever control in neurocritical care patients. The authors conclude the ArcticSun system to be superior to the Cincinnati Sub-Zero system due to the maintenance of normothermia, a higher cooling rate and better fever reduction, although shivering occurred more frequently in the ArcticSun group [18]. A surface cooling system without computer control and automatic temperature feedback is the EMCOOLS cooling system. The adhesive pads use a novel carbon cooling gel that has a high thermal conductivity resulting in a cooling rate of more than $3.5^{\circ} \mathrm{C} /$ hour. The Flex Pad needs to be adapted to the body size and shape. The feasibility trial of out-of-hospital surface cooling after return of spontaneous circulation (ROSC) in 15 survivors using the EMCOOLS system revealed a high median cooling rate of $3.3^{\circ} \mathrm{C} /$ hour, the target temperature of $33^{\circ} \mathrm{C}$ was reached approximately within 70 minutes (55 to 106 minutes) after the start of cooling and no skin lesions were observed [19]. A further novel system is the Life Recovery ThermoSuit system, which was developed mainly for fast 
Table 1(Abstract A5)

\begin{tabular}{|c|c|c|c|c|c|}
\hline Company & Device & Type of cooling & Cooling rate $\left({ }^{\circ} \mathrm{C} /\right.$ hour $)$ & Auto feedback & Reusable \\
\hline Philips & InnerCool RTx & Catheter & 4.0 to 5.0 & Yes & No \\
\hline Zoll & Thermogard XP & Catheter & 2.0 to 3.0 & Yes & No \\
\hline C.R. Bard & ArcticSun 5000 & Surface adhesive pads & 1.2 to 2.0 & Yes & No \\
\hline CSZ & Blanketrol III & Surface blanket & 1.5 & Yes & Yes \\
\hline EMCOOLS & FLEX.PAD & Surface adhesive pads & 3.5 & No & No \\
\hline MTRE & CritiCool & Surface blanket & 1.5 & Yes & no \\
\hline
\end{tabular}

The table gives the most common cooling devices with no claim to be complete. Cooling rates provided either by the company or at the company's Internet homepage. CSZ, Cincinnati Sub-Zero; MTRE, Medical ThermoRegulation Expertise.

induction of hypothermia by cold water $\left(2^{\circ} \mathrm{C}\right)$ immersion due to a lack of a temperature feedback mechanism. The water circulates continuously directly on the patient's skin with a median cooling rate of $3^{\circ} \mathrm{C} /$ hour [20]. Published data by Howes and colleagues report the safe use of the ThermoSuit system in 24 cardiac arrest survivors reaching the target temperature $\left(<34^{\circ} \mathrm{C}\right)$ within 37 minutes (range 14 to 81 minutes) [21]. After the patients have reached the target temperature, they have to be removed from the suit and cooling maintained with other methods.

Endovascular cooling: Intravascular closed-loop cooling systems are also computer controlled with a temperature feedback. The Thermogard XP Temperature Management System (Zoll) provides both a central venous catheter with an additional closed loop balloon system with circulating water for cooling. The InnerCool RTx device (Philips) using the Accutrol catheter has a special feature with an integrated temperature sensor but no additional central intravascular access. A possible advantage of taking the temperature directly in the bloodstream is the avoidance of lag in core temperature measurement inherent in rectal and bladder sensors. The very precise temperature control is needed, taking the high average cooling rates of 4.0 to $5.0^{\circ} \mathrm{C} /$ hour into consideration. This cooling system will be under evaluation in the Rapid Endovascular Catheter Core Cooling combined with cold saline as an Adjunct to Percutaneous Coronary Intervention for the Treatment of Acute Myocardial Infarction (CHILL-MI) study. This study was started in 2012 to further investigate the safety and effectiveness of the endovascular cooling system in patients suffering from ST-elevation myocardial infarction (STEMI) and to confirm the data from the Rapid-MI-ICE trial [22]. In a subanalysis of the European Hypothermia After Cardiac Arrest trial (HACA), Holzer and colleagues retrospectively reviewed the efficacy and safety of the intravascular catheter system (Cool Gard 3000, Alsius) in 56 patients, revealing a cooling rate of $1.2^{\circ} \mathrm{C} /$ hour (IQR 0.7 to 1.5) without significant differences to other techniques concerning adverse events [23]. A study by Gillies and colleagues reported a good temperature control with endovascular cooling compared with conventional ice surface cooling [24]. After induction of cooling with cold saline, one group was continued to be cooled with ice $(n=41)$ whereas the other group was cooled with the Coolgard device ( $n=42$; Alsius). In summary, catheter cooling provided a more precise temperature control, better control during rewarming, less overcooling and failure to reach target temperature. Despite these advantages there was no difference concerning outcome between both relatively small groups [24]. The duration of time an intravascular catheter can be used as central intravenous access after rewarming is not well investigated so far. Al-Senani and colleagues evaluated the safety of the lcy catheter during a cooling procedure [25]. However, intravascular catheters can cause bloodstream infections and raise the question about the risk of venous thrombosis. Few cases with thrombosis or thrombophlebitis due to a cooling catheter after a using time of respectively 7 and 10 days have been published [26]. Simosa and colleagues reported in a group of 10 patients with traumatic brain injury that five patients developing a depth venous thrombosis after an average of 5.4 days but concluded that the group under examination already had a high risk for development of thrombosis due to lack of prophylactic anticoagulation [27]. However, the approach towards anticoagulation will be different in survivors after cardiac arrest. The recommendation for the duration of use of the Icy catheter is 4 days (Icy Quattro) but a novel surface coating of the catheter material will soon be approved by authorities to enhance time of use and decrease risk of thrombosis. In addition there might be a higher risk of developing catheter-related bloodstream infections but currently no data are published studying temperature management catheters and infection rates.

Other cooling methods: The novel RhinoChill intranasal cooling device was able to demonstrate effective reduction of body temperature within the Pre-ROSC Intranasal Cooling Effectiveness trial (PRINCE trial) [28]. The portable system vaporises perfluorchlorcarbon gas with a catheter system into the nasal cavity leading to a fast induction of hypothermia first to the brain as main target organ and second to the body with a slight delay. The intra-arrest cooling approach of the study, starting induction of hypothermia already during CPR, by Castrén and colleagues was conducted as a safety and feasibility study [28]. However, benefit towards survival and neurological outcome was observed in the cooling subgroup, having received CPR within 10 minutes after collapse, although the design of the study was not conceived for outcome analysis. The randomised trial compared in detail prehospital trans-nasal cooling $(n=83)$ with advanced cardiac live support $(n=99)$ and both groups received mild hypothermia on admission to the hospital regardless of the initial rhythm. This method was able to show a significant decrease of tympanic temperature on arrival $\left(34.2^{\circ} \mathrm{C}\right.$ vs. $\left.35.5^{\circ} \mathrm{C}\right)$. Due to the convincing data from the PRINCE trial, the Prehospital Resuscitation Intra Nasal Cooling Effectiveness Survival Study (PRINCESS) started in June 2010 with patient recruitment and is designed to evaluate for good or poor neurological outcome and survival as well as to evaluate the proportion of those achieving ROSC and time to target temperature of 32 to $34^{\circ} \mathrm{C}$. First data will be available in June 2013 (ClinicalTrial.gov identifier: NCT01400373). The system has no temperature feedback and the major application area is the induction of hypothermia. Another novel approach is under investigation in the CAMARO trial (ClinicalTrials.gov identifier: NCT01016236).

Following the idea of early and fast induction of hypothermia to improve outcome and decrease side effects after cardiac arrest and incorporate novel data that hypothermia applied before a coronary intervention may reduce the infarct size in STEMI patients, a new automated peritoneal lavage system (Velomedix Inc., Palo Alto, USA) has been developed [22]. The CAMARO trial includes cardiac arrest patients as well as STEMI patients who will be cooled to a target temperature of $34^{\circ} \mathrm{C}$ without prior resuscitation. The preliminary data of this pilot study, presented as an abstract at the American Heart Association Meeting in Orlando, USA, in November 2011, showed a decrease of temperature to $34^{\circ} \mathrm{C}$ within 9 minutes, the maintenance phase of $32.5^{\circ} \mathrm{C}$ was 24 hours in cardiac arrest patients (rewarming 16 hours) and 3 hours maintenance in myocardial infarction patients (rewarming 5 hours). At the moment no device-related complication has occurred with this extremely rapid cooling method [29].

Discussion: Different cooling methods with varying technical approaches and efficacy are available to deliver mild therapeutic hypothermia to our patients. During cooling the three phases of induction, maintenance and rewarming can be defined. Are different methods necessary to fulfil the requirements in each of these three cooling phases? Taking all mentioned methods together, a combined approach seems to be the optimal way. Particularly with regard to the induction phase a combination of different methods should be suggested to increase the effectiveness of cooling, for example the combination of cold saline and a feedback cooling device, although the optimal overall timing (time to target temperature and cooling rate) is still under debate. In addition to timing, the most important question concerns shivering and its prophylactic successful treatment.

The optimal and most beneficial time point to start hypothermia after cardiac arrest is still not known. The current European resuscitation guidelines recommend starting hypothermia as soon as possible after ROSC. 
A recently published article by Sendelbach and colleagues revealed the importance of avoiding any time delay of cooling to reach good neurological outcome [30]. This 'earlier is better' strategy can be confirmed by animal data [31-34]. Following the 'earlier is better' strategy, some trials explored the possibility of inducing cooling during resuscitation or directly after ROSC, but data are controversial [35]. Induction of therapeutic hypothermia during prehospital CPR using ice-cold intravenous fluid or intranasal cooling showed that it is feasible and is partially a benefit $[28,36]$. A major problem in predicting outcome and association with timing and early cooling after cardiac arrest or even during resuscitation with these data is the small sample size and the fact that prehospital hypothermia was discontinued after admission to the hospital in many of these trials [37]. However, the analysis of data from the Scandinavian Hypothermia Network including 986 patients after cardiac arrest by Nielsen and colleagues showed no association of timing towards neurological outcome [38].

Certainly every ICU should provide $4^{\circ} \mathrm{C}$ cold saline to increase the cooling rate and to reach the target temperature as soon as possible. The administration of cold saline seems a feasible method in the preclinical setting as well as in addition to other preclinical devices available and after admission cold saline can be combined with a feedback device to speed up the cooling. Furthermore, shivering is one of the most important side effects that can occur during hypothermia leading to an increased metabolic rate, high oxygen consumption and heat generation, and therefore needs to be kept in mind to be avoided and treated aggressively. The threshold for this defence mechanism of the thermoregulatory system is around $\pm 35.5^{\circ} \mathrm{C}\left(1^{\circ} \mathrm{C}\right.$ below the vasoconstriction threshold) [39,40]. Therefore a fast induction to cross this threshold as quickly as possible seems indicated; additional treatment can include a sufficient analgosedation, magnesium and paralysation, but even the simple method of keeping the hands and feet warm by wearing socks and gloves directly from the beginning of induction of hypothermia can avoid shivering very reliably [41]. In patients with traumatic brain injury undergoing temperature management, the benefit of surface counter warming concerning less shivering and improvement of metabolic profile was reported [42]. However, a high cooling rate during induction with a combination of a feedback-cooling device and several additional conventional cooling methods in combination with hand and feet counter warming as described and a sufficient sedation level seems to be the best way to avoid shivering. In addition, every temperature management procedure requires a reliable core temperature. The gold standard is still the temperature taken directly in the bloodstream (for example, pulmonary catheter) or directly by the cooling device itself as possible with the Philips Accutrol endovascular catheter. Other common places for temperature measurement are the bladder by Foley catheters, oeosophageal probes, tympanic and rectal temperature [43]. Modern temperature management systems with high cooling rates lead to a fast induction of hypothermia that can only be detected by most temperature sensors with a time delay. The closed approach towards the gold standard might be the oesophageal measurement with an approximately average time delay of 5 minutes (range 5 to 10 minutes) [40].

Conclusion: A wide range of conventional and technical methods exists to apply mild therapeutic hypothermia after cardiac arrest. Hoedemaekers and colleagues compared all described different methods (conventional cold infusion/ice, water blankets, gel-coated pads, intravascular) in ICU patients regarding the speed of cooling $\left({ }^{\circ} \mathrm{C} /\right.$ hour $)$ and the reliability to maintain a stable target temperature. The authors conclude that water-circulating blankets, gel-coated pads and intravascular cooling are almost equally efficient for induction but intravascular methods were superior for maintaining the target temperature [44]. Some performance data might have changed over the last years due to the industry having developed the next generation of cooling devices. However, every method has its own partly limited, indication and a combination of an automatic computerprocessed feedback device with conventional methods seems a good and safe solution. The type of feedback device used in a hospital (invasive vs. non-invasive) depends on several factors but mainly on the personal preference of the treating doctors, type of patients and the local standard as well. In addition, the way of thinking is changing and it is no longer a question of making the patient cool as good as possible but rather has evolved into a complex temperature management procedure with its own risks and pitfalls as well as benefits for the patient. It is a precondition to ensure a precise and tight temperature control during all three treatment phases. Especially during rewarming, which is a very critical phase of temperature management, close temperature monitoring is necessary and can be easily achieved with a computer-feedback cooling system. A passive, uncontrolled increase of temperature should be avoided in the modern temperature management approach. However, the adoption rate and implementation of hypothermia as part of standard post-arrest care is still not high enough. Reasons are manifold but the latest version of available cooling devices may be able to help to increase the application rate by making the treatment safe and easy. If the hospital team feels confident with the topic of temperature management, numbers of operators might increase, even if the number of cardiac arrest patients treated in a hospital is low.

The presentation of different temperature-management methods and interpretation of their efficiency in the age of daily breaking news about mild hypothermia treatment and widening of the indication can only be a momentary snap-shot and cannot aspire to completeness.

Competing interests: The author received financial support and material resources from Medivance, Zoll, Philips, EMCOOL and C.R. Bard within different projects and honorarium from Medivance, Zoll and Philips for lectures. This abstract has not been influenced by anyone in collection of data, analysis, interpretation and writing.

References

1. Nolan JP, Soar J, Zideman DA, Biarent D, Bossaert LL, Deakin C, Koster RW, Wyllie J, Bottiger B: European Resuscitation Council guidelines for resuscitation 2010 Section 1. Executive summary. Resuscitation 2010, 81:1219-1276.

2. Hypothermia After Cardiac Arrest Study Group: Mild therapeutic hypothermia to improve the neurologic outcome after cardiac arrest. $N$ Engl J Med 2002, 346:549-556.

3. Bernard SA, Gray TW, Buist MD, Jones BM, Silvester W, Gutteridge G, Smith K: Treatment of comatose survivors of out-of-hospital cardiac arrest with induced hypothermia. N Engl J Med 2002, 346:557-563.

4. Hachimi-Idrissi S, Corne L, Ebinger G, Michotte Y, Huyghens L: Mild hypothermia induced by a helmet device: a clinical feasibility study. Resuscitation 2001, 51:275-281.

5. Reinikainen M, Oksanen T, Leppanen P, Torppa T, Niskanen M, Kurola J: Mortality in out-of-hospital cardiac arrest patients has decreased in the era of therapeutic hypothermia. Acta Anaesthesiol Scand 2012, 56:110-115.

6. Kim F, Olsufka M, Carlbom D, Deem S, Longstreth WT, Hanrahan M, Maynard C, Copass MK, Cobb LA: Pilot study of rapid infusion of $2 \mathrm{~L}$ of $4^{\circ} \mathrm{C}$ normal saline for induction of mild hypothermia in hospitalized, comatose survivors of out-of-hospital cardiac arrest. Circulation 2005, 112:715-719.

7. Larsson IM, Wallin E, Rubertsson S: Cold saline infusion and ice packs alone are effective in inducing and maintaining therapeutic hypothermia after cardiac arrest. Resuscitation 2010, 81:15-19.

8. Spiel AO, Kliegel A, Janata A, Uray T, Mayr FB, Laggner AN, Jilma B, Sterz F: Hemostasis in cardiac arrest patients treated with mild hypothermia initiated by cold fluids. Resuscitation 2009, 80:762-765.

9. Virkkunen I, Yli-Hankala A, Silfvast T: Induction of therapeutic hypothermia after cardiac arrest in prehospital patients using ice-cold Ringer's solution: a pilot study. Resuscitation 2004, 62:299-302.

10. Kliegel A, Losert H, Sterz F, Kliegel M, Holzer M, Uray T, Domanovits H: Cold simple intravenous infusions preceding special endovascular cooling for faster induction of mild hypothermia after cardiac arrest - a feasibility study. Resuscitation 2005, 64:347-351.

11. Storm C, Schefold JC, Kerner T, Schmidbauer W, Gloza J, Krueger A, Jorres A, Hasper D: Prehospital cooling with hypothermia caps (PreCoCa): a feasibility study. Clin Res Cardiol 2008, 97:768-772.

12. Kim F, Olsufka M, Longstreth WT, Maynard C, Carlbom D, Deem S, Kudenchuk P, Copass MK, Cobb LA: Pilot randomized clinical trial of prehospital induction of mild hypothermia in out-of-hospital cardiac arrest patients with a rapid infusion of 4 degrees $C$ normal saline. Circulation 2007, 115:3064-3070

13. Kliegel A, Janata A, Wandaller C, Uray T, Spiel A, Losert H, Kliegel M, Holzer M, Haugk M, Sterz F, Laggner AN: Cold infusions alone are effective for induction of therapeutic hypothermia but do not keep patients cool after cardiac arrest. Resuscitation 2007, 73:46-53.

14. Heard KJ, Peberdy MA, Sayre MR, Sanders A, Geocadin RG, Dixon SR, Larabee TM, Hiller K, Fiorello A, Paradis NA, O'Neil BJ: A randomized controlled trial comparing the Arctic Sun to standard cooling for induction of hypothermia after cardiac arrest. Resuscitation 2010, 81:9-14. 
15. Tomte O, Draegni T, Mangschau A, Jacobsen D, Auestad B, Sunde K: A comparison of intravascular and surface cooling techniques in comatose cardiac arrest survivors. Crit Care Med 2011, 39:443-449.

16. Varon J, Acosta P, Wintz R, Mendoza N: Unusual side effect from hydrogel pads during therapeutic hypothermia. Resuscitation 2008, 78:248-249.

17. Laish-Farkash A, Matetzky S, Kassem S, Haj-lahia H, Hod H: Therapeutic hypothermia for comatose survivors after cardiac arrest. Israel Med Assoc J 2007, 9:252-256.

18. Mayer SA, Kowalski RG, Presciutti M, Ostapkovich ND, McGann E, Fitzsimmons BF, Yavagal DR, Du YE, Naidech AM, Janjua NA, Claassen J, Kreiter KT, Parra A, Commichau C: Clinical trial of a novel surface cooling system for fever control in neurocritical care patients. Crit Care Med 2004, 32:2508-2515.

19. Uray T, Malzer R: Out-of-hospital surface cooling to induce mild hypothermia in human cardiac arrest: a feasibility trial. Resuscitation 2008, 77:331-338.

20. Janata A, Weihs W, Bayegan K, Schratter A, Holzer M, Behringer W, Schock RB, Losert UM, Springler G, Schmidt P, Sterz F: Therapeutic hypothermia with a novel surface cooling device improves neurologic outcome after prolonged cardiac arrest in swine. Crit Care Med 2008, 36:895-902.

21. Howes D, Ohley W, Dorian P, Klock C, Freedman R, Schock R, Krizanac D, Holzer M: Rapid induction of therapeutic hypothermia using convectiveimmersion surface cooling: safety, efficacy and outcomes. Resuscitation 2010, 81:388-392.

22. Gotberg M, Olivecrona GK, Koul S, Carlsson M, Engblom H, Ugander M, van der Pals J, Algotsson L, Arheden H, Erlinge D: A pilot study of rapid cooling by cold saline and endovascular cooling before reperfusion in patients with ST-elevation myocardial infarction. Circ Cardiovasc Interv 2010, 3:400-407

23. Holzer M, Mullner M, Sterz F, Robak O, Kliegel A, Losert H, Sodeck G, Uray T, Zeiner A, Laggner AN: Efficacy and safety of endovascular cooling after cardiac arrest: cohort study and Bayesian approach. Stroke 2006, 37:1792-1797.

24. Gillies MA, Pratt R, Whiteley C, Borg J, Beale RJ, Tibby SM: Therapeutic hypothermia after cardiac arrest: a retrospective comparison of surface and endovascular cooling techniques. Resuscitation 2010, 81:1117-1122.

25. Al-Senani FM, Graffagnino C, Grotta JC, Saiki R, Wood D, Chung W, Palmer G, Collins KA: A prospective, multicenter pilot study to evaluate the feasibility and safety of using the CoolGard System and Icy catheter following cardiac arrest. Resuscitation 2004, 62:143-150.

26. Prunet B, Lacroix G, Bordes J, Poyet R, D'Aranda E, Goutorbe P: Catheter related venous thrombosis with cooling and warming catheters: two case reports. Cases J 2009, 2:8857.

27. Simosa HF, Petersen DJ, Agarwal SK, Burke PA, Hirsch EF: Increased risk of deep venous thrombosis with endovascular cooling in patients with traumatic head injury. Am Surg 2007, 73:461-464.

28. Castren M, Nordberg P, Svensson L, et al: Intra-arrest transnasal evaporative cooling: a randomized, prehospital, multicenter study (PRINCE: Pre-ROSC IntraNasal Cooling Effectiveness). Circulation 2010, 122:729-736.

29. Polderman $K$, CAMARO trialists: CAMARO trial, preliminary results on safety [abstract 13265]. Circulation 2011, 124.

30. Sendelbach S, Hearst MO, Johnson PJ, Unger BT, Mooney MR: Effects of variation in temperature management on cerebral performance category scores in patients who received therapeutic hypothermia post cardiac arrest. Resuscitation 2012 in press.

31. Kuboyama K, Safar P, Radovsky A, Tisherman SA, Stezoski SW, Alexander H: Delay in cooling negates the beneficial effect of mild resuscitative cerebral hypothermia after cardiac arrest in dogs: a prospective, randomized study. Crit Care Med 1993, 21:1348-1358.

32. Nozari A, Safar P, Stezoski SW, Wu X, Kostelnik S, Radovsky A, Tisherman S, Kochanek PM: Critical time window for intra-arrest cooling with cold saline flush in a dog model of cardiopulmonary resuscitation. Circulation 2006, 113:2690-2696.

33. Jia X, Koenig MA, Shin HC, Zhen G, Pardo CA, Hanley DF, Thakor NV, Geocadin RG: Improving neurological outcomes post-cardiac arrest in a rat model: immediate hypothermia and quantitative EEG monitoring. Resuscitation 2008, 76:431-442.

34. Colbourne F, Corbett D: Delayed postischemic hypothermia: a six month survival study using behavioral and histological assessments of neuroprotection. J Neurosci 1995, 15:7250-7260.
35. Kamarainen A, Virkkunen I, Tenhunen J, Yli-Hankala A, Silfvast T: Prehospital therapeutic hypothermia for comatose survivors of cardiac arrest: a randomized controlled trial. Acta Anaesthesio/ Scand 2009, 53:900-907.

36. Kamarainen A, Virkkunen I, Tenhunen J, Yli-Hankala A, Silfvast T: Prehospital induction of therapeutic hypothermia during CPR: a pilot study. Resuscitation 2008, 76:360-363.

37. Kamarainen A, Hoppu S, Silfvast T, Virkkunen I: Prehospital therapeutic hypothermia after cardiac arrest - from current concepts to a future standard. Scand J Trauma Resusc Emerg Med 2009, 17:53.

38. Nielsen N, Hovdenes J, Nilsson F, Rubertsson S, Stammet P, Sunde K, Valsson F, Wanscher M, Friberg $\mathrm{H}$ : Outcome, timing and adverse events in therapeutic hypothermia after out-of-hospital cardiac arrest. Acta Anaesthesiol Scand 2009, 53:926-934.

39. Lopez M, Sessler DI, Walter K, Emerick T, Ozaki M: Rate and gender dependence of the sweating, vasoconstriction, and shivering thresholds in humans. Anesthesiology 1994, 80:780-788.

40. Polderman $\mathrm{KH}$, Herold I: Therapeutic hypothermia and controlled normothermia in the intensive care unit: practical considerations, side effects, and cooling methods. Crit Care Med 2009, 37:1101-1120.

41. van Zanten AR, Polderman KH: Blowing hot and cold? Skin counter warming to prevent shivering during therapeutic cooling. Crit Care Med 2009, 37:2106-2108.

42. Badjatia N, Strongilis E, Prescutti M, Fernandez L, Fernandez A, Buitrago M Schmidt JM, Mayer SA: Metabolic benefits of surface counter warming during therapeutic temperature modulation. Crit Care Med 2009, 37:1893-1897.

43. Hasper D, Nee J, Schefold JC, Krueger A, Storm C: Tympanic temperature during therapeutic hypothermia. Emerg Med J 2011, 28:483-485.

44. Hoedemaekers CW, Ezzahti M, Gerritsen A, van der Hoeven JG: Comparison of cooling methods to induce and maintain normo- and hypothermia in intensive care unit patients: a prospective intervention study. Crit Care 2007, 11:R91.

A6

Pharmacodynamics in hypothermia

Torkjel Tveita ${ }^{1,2}$

${ }^{1}$ Anesthesia and Critical Care, Institute of Clinical Medicine, University of

Tromsø, Norway; ${ }^{2}$ University Hospital of Northern Norway, Tromsø, Norway

Critical Care 2012, 16(Suppl 2):A6

Introduction: Guidelines for using inotropic drugs to support cardiovascular function at low core temperatures are not well characterized. The safe application of inotropic drugs during normothermic conditions, routinely used to treat cardiovascular instability by effectively increasing cardiac output (CO) and improve end-organ perfusion associated with acute heart failure [1], is based on a detailed understanding of pharmacodynamics and pharmacokinetics of these drugs. Detailed knowledge of temperaturedependent changes in pharmacodynamics and pharmacokinetics of such cardioactive drugs is essential for establishing treatment guidelines.

Therapeutic hypothermia: Over the last decade therapeutic hypothermia has been established as a recognized intervention to increase survival and improve neurologic outcome in adult comatose cardiac arrest survivors [2-6]. However, after return of spontaneous circulation (ROSC) and coronary revascularization, more than $50 \%$ of survivors suffer from acute heart failure and need inotropic cardiac support to resume adequate circulatory function [2] after induction of hypothermia when the core temperature is deliberately lowered to 34 to $32^{\circ} \mathrm{C}$ and maintained for 24 to 48 hours. In contrast, in patients hospitalized for acute heart failure without hypothermia a subgroup of about only $10 \%$ received inotropic drugs [7].

Accidental hypothermia: Another group of patients in need of inotropic drug therapy at low core temperature are accidental hypothermia patients displaying hypothermia-induced cardiac failure during rewarming, ranging from mild reduction of $\mathrm{CO}$ to the fulminant circulatory shock termed rewarming shock [8-11]. Rewarming shock is a clinically descriptive term that refers to a pathophysiologic state of cardiovascular collapse taking place during or after rewarming from accidental hypothermia [12], recognized as a progressive reduction of $\mathrm{CO}$ and a sudden fall in arterial blood pressure. In order to treat or prevent rewarming shock, cardioactive inotropic drugs are commonly necessary to elevate a low CO.

Research in experimental hypothermia has displayed a substantial depression of LV myocardial function in earlier studies $[8,11,13]$ as well as 
in recent studies $[9,13,14]$. Based on the results from these studies, cellular calcium overload, disturbed calcium homeostasis, changes in myocardial myofilament responsiveness to intracellular calcium as well as impaired high-energy phosphate homeostasis could all be proposed as important factors leading to the changes observed in the hypothermic heart and contributing to failure of functional recovery during rewarming [15-18].

$\beta$-receptor agonists: In the acutely failing heart postoperatively, only drugs such as epinephrine and NE provide positive inotropy and perfusion pressure. Epinephrine acts through stimulation via sarcolemmal $\beta$ adrenoceptors, causing phosphorylation of the sarcolemmal L-type $\mathrm{Ca}^{2+}$ channel via cyclic AMP and protein kinase A pathways. This phosphorylation increases the open probability of the channel [19], allowing for greater trans-sarcolemmal $\mathrm{Ca}^{2+}$ influx with each depolarization and producing, in part, the positive inotropic effect of epinephrine.

Vascular effects: Only a few experiments studying effects of epinephrine during hypothermia or/and rewarming using in vivo animal models have been published. Some authors report that both $\beta_{1}$-adrenoceptors and $\alpha$ adrenoceptors increase their sensitivity to catecholamines during hypothermia [18,20-22] as $\beta_{1}$-adrenoceptor activity was potentiated by low temperature, and they claim the existence of hypothermia-induced supersensitivity and increased agonist activity for $\beta_{1}$-adrenoceptors. In support of this view, a left shift of the concentration-response curve for epinephrine during hypothermia has been reported [23]. However, others suggest hypothermia-induced increase in sensitivity for both $\alpha_{1}{ }^{-}$ adrenoceptors and $\alpha_{2}$-adrenoceptors during cooling [24], but that sensitivity of $\beta_{1}$-adrenoceptors is not increased to the same extent as $\alpha$-adrenoceptors. In contrast, other researchers have reported a hypothermia-induced supersensitivity of $\beta_{1}$-adrenoceptors [18,20,21]. Rubinstein reported that hypothermia modified the vascular response to epinephrine [25]; that is, the epinephrine doses that induced vasodilation during normothermic conditions increased TPR at $25^{\circ} \mathrm{C}$. He also claimed that myocardial contractile effects of epinephrine is reduced at low temperatures (covered below), a view also supported by others [25,25-27]. Experimental data show that the sympathetic nervous system could be switched off at a threshold temperature about $29^{\circ} \mathrm{C}$ and hypotensive patients with temperatures below this may benefit from infusions of exogenous catecholamines [28]. In addition, if $\mathrm{CO}$ could be elevated pharmacologically, rewarming by any means becomes more efficient $[17,29]$. Some researchers recommend infusion of low doses of catecholamines in patients who have lower blood pressure than would be expected for that degree of hypothermia and who are not responding to crystalloids and rewarming [29].

It therefore seems that the use of vasoactive drugs during hypothermic conditions remains quite contradictory.

Cardiac effects: Some sources claim that the hypothermic heart is unresponsive [30] or little responsive [31] to cardioactive drugs, and the last reference as well as recommendation of the American Heart Association [26] refer to the potential hazard of overmedication, due to delayed drug metabolism leading to accumulation to toxic levels in patients suffering from deep or severe hypothermia, if used repeatedly. The AHA recommends the following algorithm for treatment of hypothermia: below $30^{\circ} \mathrm{C}$ i.v. epinephrine should not be given, but above $30^{\circ} \mathrm{C}$ epinephrine should be given, if indicated, but at longer than standard intervals. To date, there are no prospective clinical studies to support the recommendation to avoid epinephrine during hypothermic CPR, but a preclinical work report major side effects of repeated epinephrine administration during experimental hypothermic CPR in pigs [32]. Even in the last recommendations from the AHA it was stated that treatment of severe hypothermia (temperature $<30^{\circ} \mathrm{C}$ ) in the field remains controversial [26].

A reduction of $\mathrm{CO}$ and SV by inducing varying levels of hypercalcaemia during hypothermia $\left(28^{\circ} \mathrm{C}\right)$ was reported by Schiffmann and colleagues [18]. Following infusion of epinephrine during these experimental conditions, CO and SV were even more depressed [18]. These findings shear similarities with findings in our intact animal models: infusion of epinephrine, which theoretically will induce opening of $\mathrm{Ca}^{2+}$ channels, increase calcium influx and elevate intracellular calcium even further, caused a significant depression of myocardial function during hypothermic as well as posthypothermic conditions $[13,33,34]$. This made us conclude that hypothermia and rewarming may cause alterations in the pharmacodynamic effects of $\alpha$ receptor and $\beta$-receptor mediated drugs $[13,33,35-37]$ or induce changes in receptor affinity for these drugs. Further, we found that low-dose epinephrine managed to maintain positive inotropic effects on LV cardiac function during cooling to $30^{\circ} \mathrm{C}$, but these effects vanished during cooling to $28^{\circ} \mathrm{C}$. If the epinephrine dose was increased at these low temperatures SV and $\mathrm{CO}$ were not elevated but a significant increase in afterload took place. Thus we conclude that if epinephrine is applied during hypothermia the therapeutic window appears narrow with short distance to unwanted side effects. Further, the prehypothermic dose-dependent increase in LV function in response to epinephrine was not present after rewarming in the group that had received epinephrine during hypothermia [13,33,35,38], and if epinephrine was infused during rewarming vascular side effects of epinephrine (vasoconstriction) dominated $[13,38]$ without elevating CO above pre-hypothermic control values at any temperature.

During cooling there is a reduction in $\mathrm{Ca}^{2+}$ sensitivity of troponin $\mathrm{C}$ due to protein kinase A (PKA)-induced phosphorylation of troponin I [39], in addition to the hypothermia-induced calcium overload $[18,19]$. Further, it is documented that PKA may inhibit the activity of adenylyl cyclase (AC) 5 [40,41], predominantly expressed in the heart [42], which catalyzes ATP to CAMP. Based on this information, it is possible that lack of positive inotropic effect on cardiac function of $\beta$-receptor stimulation during hypothermia may be due to the inhibitory effects on AC 5 activity of PKA phosphorylation and/or a potential desensitization and internalization of the $\beta$-adrenoceptor. Another way of interpreting these results is that the efficacy of signal transduction through G-protein coupled receptors is rapidly decreased through mechanisms like desensitization and internalization, mechanisms that will avoid receptor overstimulation. However, during pathologic conditions, like acute heart failure following acute coronary syndromes and cardiac standstill, or as a consequence of exposure to accidental hypothermia, it is necessary for cardiac myocytes to produce CAMP over the limitation of such adaptations.

Dopamine: The still widespread use of DA in perioperative and intensive care, the explicit recommendation for its use in accidental hypothermia guidelines, and possible positive effects in hypothermia as reported from experimental studies all need evaluation. From experimental hypothermia research we found that DA improved both systolic and diastolic function in hypothermia [43]. However, at $25^{\circ} \mathrm{C}$ no beneficial effect was seen on $\mathrm{Cl}$ as $\mathrm{SI}$ decreased with incrementing DA dosages. Increased SVRI at high-dose DA at $25^{\circ} \mathrm{C}$ suggests $\alpha$-adrenergic involvement not seen in normothermia. Properties of the low-flow, high-viscosity circulatory state, combined with serious alterations in the pharmacokinetics of DA, may explain lack of beneficial - and potentially harmful - effects from DA administration at $25^{\circ} \mathrm{C}$. Milrinone: Milrinone is a phosphodiesterase (PDE) 3 inhibitor that is dominantly expressed in the heart and vascular tissues. The site of action of milrinone is cytosolic, and the administration of a PDE3 inhibitor increases CAMP only in the cardiovascular system, which subsequently enhance cardiac contraction and induce vasodilation during normothermic conditions [44]. In a recent experiment [45], using milrinone as a model drug of intracellular mode of action, the positive cardiac effects of this drug was demonstrated during normothermic conditions (30\% increase in SV and CO) and remained during cooling to $15^{\circ} \mathrm{C}$. These effects of milrinone on cardiac function stay in essential contrast to those in our previous studies testing effects of $\alpha$-receptor and $\beta$-receptor mediated drugs $[35,38]$ during cooling and rewarming.

Conclusion: Taken together, the use of cardioactive drugs during hypothermic conditions remains quite contradictory. Therefore, pharmacologic treatment applied during the clinically challenging modalities, therapeutic as well as accidental hypothermia, call upon written treatment protocols or guidelines that are so far largely missing or at least not properly updated. More research is needed to explore temperature-dependent changes in pharmacodynamics and pharmacokinetics of cardioactive drugs to write these guidelines. Thus, due to significant hypothermia-induced alterations of cardiac as well as vascular adrenoceptor sensitivity, the use of cardioactive agents not affecting these receptor systems are advised during hypothermic conditions.

References

1. Petersen JW, Felker GM: Inotropes in the management of acute heart failure. Crit Care Med 2008, 36:S106-S111.

2. Bernard SA, Gray TW, Buist MD, Jones BM, Silvester W, Gutteridge G, Smith K: Treatment of comatose survivors of out-of-hospital cardiac arrest with induced hypothermia. N Engl J Med 2002, 346:557-563.

3. Safar PJ, Kochanek PM: Therapeutic hypothermia after cardiac arrest. N Engl J Med 2002, 346:612-613.

4. Polderman $\mathrm{KH}$ : Mechanisms of action, physiological effects, and complications of hypothermia. Crit Care Med 2009, 37:S186-S202. 
5. Polderman $\mathrm{KH}$ : Induced hypothermia and fever control for prevention and treatment of neurological injuries. Lancet 2008, 371:1955-1969.

6. Polderman $\mathrm{KH}$ : Hypothermia and neurological outcome after cardiac arrest: state of the art. Eur J Anaesthesiol Suppl 2008, 42:23-30.

7. Abraham WT, Adams KF, Fonarow GC, Costanzo MR, Berkowitz RL, LeJemtel TH, Cheng ML, Wynne J: In-hospital mortality in patients with acute decompensated heart failure requiring intravenous vasoactive medications: an analysis from the Acute Decompensated Heart Failure National Registry (ADHERE). J Am Coll Cardiol 2005, 46:57-64.

8. Blair $\mathrm{E}$, Montgomery AV, Swan $\mathrm{H}$ : Posthypothermic circulatory failure. I. Physiologic observations on the circulation. Circulation 1956, 13:909-915.

9. Kondratiev TV, Flemming K, Myhre ES, Sovershaev MA, Tveita T: Is oxygen supply a limiting factor for survival during rewarming from profound hypothermia? Am J Physiol Heart Circ Physiol 2006, 291:H441-H450.

10. Patton JF, Doolittle WH: Core rewarming by peritoneal dialysis following induced hypothermia in the dog. J Appl Physiol 1972, 33:800-804.

11. Tveita T, Skandfer M, Refsum H, Ytrehus K: Experimental hypothermia and rewarming: changes in mechanical function and metabolism of rat hearts. J Appl Physiol 1996, 80:291-297.

12. Maclean D, Emslie-Smith D: Accidental Hypothermia Melbourne: Blackwell Scientific 1977.

13. Kondratiev TV, Myhre ES, Simonsen O, Nymark TB, Tveita T: Cardiovascular effects of epinephrine during rewarming from hypothermia in an intact animal model. J Appl Physiol 2006, 100:457-464.

14. Filseth OM, How OJ, Kondratiev T, Gamst TM, Tveita T: Post-hypothermic cardiac left ventricular systolic dysfunction after rewarming in an intact pig model. Crit Care 2010, 14:R211.

15. Baller D, Wolpers HG, Schrader R, Hoeft A, Korb H, Rosick A, Bretschneider HJ, Hellige G: Paradoxical effects of catecholamines and calcium on myocardial function in moderate hypothermia. Thorac Cardiovasc Surg 1983, 31:131-138.

16. Belke DD, Wang LC, Lopaschuk GD: Effects of hypothermia on energy metabolism in rat and Richardson's ground squirrel hearts. J Appl Physiol 1997, 82:1210-1218.

17. Nicodemus HF, Chaney RD, Herold R: Hemodynamic effects of inotropes during hypothermia and rapid rewarming. Crit Care Med 1981, 9:325-328.

18. Schiffmann H, Gleiss J, von HA, Schroder T, Kahles H, Hellige G: Effects of epinephrine on the myocardial performance and haemodynamics of the isolated rat heart during moderate hypothermia - importance of calcium homeostasis. Resuscitation 2001, 50:309-317.

19. Bers DM: Ca regulation in cardiac muscle. Med Sci Sports Exerc 1991 23:1157-1162.

20. Dundee JW, Clarke RS: pharmacology of hypothermia. Int Anesthesiol Clin 1964, 2:857-872.

21. Williams RG, Broadley KJ: Responses mediated via beta 1, but not beta 2adrenoceptors, exhibit hypothermia-induced supersensitivity. Life Sci 1982, 31:2977-2983.

22. Chess Williams RG, Broadley KJ, Duncan C: A fundamental temperaturedependent difference between beta-adrenoceptor agonists and antagonists. Life Sci 1984, 35:1091-1099.

23. Riishede L, Nielsen-Kudsk F: Myocardial effects of adrenaline, isoprenaline and dobutamine at hypothermic conditions. Pharmacol Toxicol 1990 66:354-360.

24. Gomez B, Borbujo J, Garcia-Villalon AL, Nava-Hernandez E, Valle J, Garcia JL, Dieguez G: Alpha 1- and alpha 2-adrenergic response in human isolated skin arteries during cooling. Gen Pharmacol 1991, 22:341-346.

25. Rubinstein $\mathrm{EH}$ : Vascular responses to adrenaline, noradrenaline and angiotensin in hypothermic dogs. Acta Physiol Lat Am 1961, 11:30-37.

26. Guidelines for cardiopulmonary resuscitation and emergency cardiac care. Emergency Cardiac Care Committee and Subcommittees, American Heart Association. Part IV. Special resuscitation situations. JAMA 1992, 268:2242-2250.

27. Omar SA, Hammad D, Varma S: Reduced beta adrenergic responsiveness in isolated rabbit atria during hypothermia. Indian J Physiol Pharmacol 1979, 23:199-203.

28. Chernow B, Lake CR, Zaritsky A, Finton CK, Casey L, Rainey TG, Fletcher JR: Sympathetic nervous system 'switch off' with severe hypothermia. Crit Care Med 1983, 11:677-680

29. Danzl DF, Pozos RS: Accidental hypothermia. N Engl J Med 1994, 331:1756-1760

30. Lloyd EL: Accidental hypothermia. Resuscitation 1996, 32:111-124.

31. Murray P, Hall J: Principles of Critical Care New York: McGraw-Hill 1998.
32. Kornberger E, Lindner KH, Mayr VD, Schwarz B, Rackwitz KS, Wenzel V, Krismer AC, Mair P: Effects of epinephrine in a pig model of hypothermic cardiac arrest and closed-chest cardiopulmonary resuscitation combined with active rewarming. Resuscitation 2001, 50:301-308.

33. Kondratiev TV, Tveita T: Effects of sympathetic stimulation during cooling on hypothermic as well as posthypothermic hemodynamic function. Can J Physiol Pharmacol 2006, 84:985-991.

34. Kondratiev TV, Wold RM, Aasum E, Tveita T: Myocardial mechanical dysfunction and calcium overload following rewarming from experimental hypothermia in vivo. Cryobiology 2008, 56:15-21.

35. Han YS, Tveita T, Kondratiev TV, Prakash YS, Sieck GC: Changes in cardiovascular beta-adrenoceptor responses during hypothermia. Cryobiology 2008, 57:246-250.

36. Mortensen E, Berntsen R, Tveita T, Lathrop DA, Refsum H: Changes in ventricular fibrillation threshold during acute hypothermia: a model for future studies. J Basic Clin Physiol Pharmacol 1993, 4:313-319.

37. Bokenes L, Alexandersen TE, Osterud B, Tveita T, Mercer JB: Physiological and haematological responses to cold exposure in the elderly. Int $J$ Circumpolar Health 2000, 59:216-221.

38. Tveita T, Sieck GC: The physiologic responses to epinephrine during cooling and after rewarming in vivo. Crit Care 2011, 15:R225.

39. Han YS, Tveita T, Prakash YS, Sieck GC: Mechanisms underlying hypothermia-induced cardiac contractile dysfunction. Am J Physiol Heart Circ Physiol 2010, 298:H890-H897.

40. Iwami G, Kawabe J, Ebina T, Cannon PJ, Homcy CJ, Ishikawa Y: Regulation of adenylyl cyclase by protein kinase A. J Biol Chem 1995, 270:12481-12484.

41. Chen Y, Harry A, Li J, Smit MJ, Bai X, Magnusson R, Pieroni JP, Weng G, lyengar R: Adenylyl cyclase 6 is selectively regulated by protein kinase A phosphorylation in a region involved in Galphas stimulation. Proc Natl Acad Sci USA 1997, 94:14100-14104.

42. Iwatsubo K, Okumura S, Ishikawa Y: Drug therapy aimed at adenylyl cyclase to regulate cyclic nucleotide signaling. Endocr Metab Immune Disord Drug Targets 2006, 6:239-247

43. Filseth OM, How OJ, Kondratiev T, Gamst TM, Sager G, Tveita T: Changes in cardiovascular effects of dopamine in response to graded hypothermia in vivo. Crit Care Med 2012, 40:178-186.

44. Iwatsubo K, Okumura S, Ishikawa Y: Drug therapy aimed at adenylyl cyclase to regulate cyclic nucleotide signaling. Endocr Metab Immune Disord Drug Targets 2006, 6:239-247.

45. Tveita, Sieck GC: Effects of milrinone on left ventricular cardiac function during cooling in an intact animal model. Cryobiology 2012 in press.

\section{HOT AND COLD}

\section{A7}

Pathogenetic mechanisms of heatstroke and novel therapies

Abderrezak Bouchama

King Abdullah International Medical Research Center, Riyadh, Kingdom of

Saudi Arabia

Critical Care 2012, 16(Suppl 2):A7

Heatstroke, a life-threatening condition defined by a rapidly increasing core temperature greater than $40^{\circ} \mathrm{C}$ and multiple organ system dysfunction, is a leading cause of morbidity and mortality during heat waves [1]. The heat wave that affected Europe during August 2003 led to an unprecedented 70,000 excess deaths of which up to $40 \%$ were confirmed to be due to heatstroke [2]. Sophisticated climate models predict increasing frequency and severity of heat waves and so the incidence of heat-related death could rise if proactive measures and novel therapy to address this threat are not adopted [3]

The mechanisms of multiple organ system dysfunction in heatstroke are not fully understood and include direct tissue injury and cell death resulting from heat cytotoxicity together with delayed organ dysfunction and damage secondary to activation of inflammatory and coagulation pathways. Histopathological changes include endothelial injury, disseminated intravascular thrombosis, neutrophils infiltration and apoptosis [4,5]. Despite cooling and optimal treatment in intensive care the overall mortality from heatstroke exceeds $60 \%$, due in large part to the fact that no specific treatment is available [6,7].

Experimental evidence from rodent models of heatstroke suggest that immunomodulation of the host response may alter the clinical course of 
heatstroke and thereby improve outcome [8-10]. Heatstroke in induces systemic and local (central nervous system) production of TNF $\alpha$ and IL-1, and severe coagulopathy. This is associated with multiple organ system dysfunction including severe neuronal injury, and high mortality. Administration of an IL-1 receptor antagonist [8], corticosteroids [9] or activated protein $C$ [10] before the onset of heatstroke attenuates the multiple organ system dysfunction and improves survival. However, extrapolation of these data from these small animal models to humans is problematic because of inter-species differences.

Baboons represent the most appropriate model for the study of the host inflammatory and hemostatic responses to heat stress and their relation to cellular injury and death as well as for testing novel therapy, which targets these pathways. Moreover, the findings may have direct applicability to human heatstroke, and could represent the basis for clinical trial. In this review, we show that baboons subjected to heat stress reproduce both the clinical and immunological changes similar to those seen in humans [11]. Using this experimental model, we report that immunomodulating the host systemic inflammatory and coagulation responses failed to translate into improved survival, suggesting that further basic research on the pathogenesis of heatstroke is required [12-14].

\section{References}

1. Bouchama A, Knochel JP: Heat stroke. N Engl J Med 2002, 346:1978-1988.

2. Robine JM, Cheung SL, Le Roy S, Van Oyen H, Griffiths C, Michel JP,

Herrmann FR: Death toll exceeded 70,000 in Europe during the summer of 2003. C R Biol 2008, 331:171-178.

3. Easterling DR, Meehl GA, Parmesan C, Changnon SA, Karl TR, Mearns LO: Climate extremes: observations, modeling, and impacts. Science 2000, 289:2068-2074.

4. Malamud N, Haymaker W, Custer R: Heatstroke: a clinico-pathologic study of 125 fatal cases. Milit Surg 1946, 99:397-449.

5. Chao TC, Sinniah R, Pakiam JE: Acute heat stroke deaths. Pathology 1981, 13:145-156.

6. Misset B, De Jonghe B, Bastuji-Garin S, Gattolliat O, Boughrara E, Annane D, Hausfater P, Garrouste-Orgeas M, Carlet J: Mortality of patients with heatstroke admitted to intensive care units during the 2003 heat wave in France: a national multiple-center risk-factor study. Crit Care Med 2006, 34:1087-1092.

7. Argaud L, Ferry T, Le QH, Marfisi A, Ciorba D, Achache P, Ducluzeau R, Robert D: Short- and long-term outcomes of heatstroke following the 2003 heat wave in Lyon, France. Arch Intern Med 2007, 167:2177-2183.

8. Lin MT, Liu HH, Yang YL: Involvement of interleukin-1 receptor mechanisms in development of arterial hypotension in rat heatstroke. Am J Physiol 1997, 273(4 Pt 2):H2072-H2077.

9. Liu CC, Chien CH, Lin MT: Glucocorticoids reduce interleukin-1 concentration and result in neuroprotective effects in rat heatstroke. J Physiol 2000, 527(Pt 2):333-343.

10. Chen CM, Hou CC, Cheng KC, Tian RL, Chang CP, Lin MT: Activated protein C therapy in a rat heat stroke model. Crit Care Med 2006, 34:1960-1966.

11. Bouchama A, Roberts G, Al Mohanna F, El-Sayed R, Lach B, Chollet-Martin S, Ollivier V, Al Baradei R, Loualich A, Nakeeb S, et al: Inflammatory, hemostatic, and clinical changes in a baboon experimental model for heatstroke. J Appl Physiol 2005, 98:697-705.

12. Bouchama A, Kwaasi A, Dehbi M, Al Mohanna F, Eldali A, El-Sayed R, Tbakhi A, Alzahrani AS, Roberts AG: Glucocorticoids do not protect against the lethal effects of experimental heatstroke in baboons. Shock 2007, 27:578-583.

13. Bouchama A, Kunzelmann C, Dehbi M, Kwaasi A, Eldali A, Zobairi F, Freyssinet JM, de Prost D: Recombinant activated protein $C$ attenuates endothelial injury and inhibits procoagulant microparticles release in baboon heatstroke. Arterioscler Thromb Vasc Biol 2008, 28:1318-1325.

14. Bouchama A, Al-Mohanna F, Assad L, Baturcam E, Eldali A, Owaidah T, et al: Tissue factor/factor Vlla pathway mediates coagulation activation in induced-heat stroke in the baboon. Crit Care Med 2011, 40:1229-1236.

A8

Is therapeutic hypothermia immunosuppressive?

Kees $\mathrm{H}$ Polderman

Department of Critical Care Medicine, University of Pittsburgh Medical

Center, Pittsburgh, PA, USA

Critical Care 2012, 16(Suppl 2):A8
Introduction: The answer to the question in the title of this article would appear obvious. Even the term 'to catch a cold' is partly based on the widely-held assumption that lower temperatures or a chill can decrease the resistance to certain viral infections such as the common cold [1]. Furthermore, a number of studies have clearly demonstrated that inadvertent decreases in temperature in the perioperative setting, and in some other situations, can significantly increase infection risk.

However, the question is not as easy to answer in cases where hypothermia is applied deliberately and with various precautions, which is the case in therapeutic cooling. In these cases potentially harmful responses such as shivering and tachycardia are carefully controlled, body temperatures are not allowed to drop below a predefined setpoint, and every effort is made to prevent side effects [2,3]. Moreover, even the evidence that hypothermia plays a role in the development of the common cold is mixed, and the few studies performed to address this issue did not support the popular belief that colds are associated with exposure to a cold environment $[1,4]$.

The relationship between hypothermia and immune function are briefly discussed below.

In vitro studies and animal experiments: In most types of acute brain injury there is a significant and protracted inflammatory response in the hours following the acute event, whether this is ischemic, traumatic, or a combination of both. Proinflammatory mediators such as TNF $\alpha$ and IL-1 are released in large quantities by astrocytes, microglia, and endothelial cells following an episode of ischemia and reperfusion [2]. The levels of these mediators begin to rise around 1 hour after injury and remain elevated for up to 5 days $[2,5,6]$. This in turn stimulates the chemotaxis of activated leucocytes across the blood-brain barrier and leads to an accumulation of inflammatory cells in the injured brain, as well as the emergence of adhesion molecules on leukocytes and endothelial cells. Simultaneously activation of the complement system occurs, beginning in the very early stages after brain injury. This further stimulates the passage of neutrophils and (in later stages) monocytes/macrophages [6]. These inflammatory and immunological responses occur especially during reperfusion and are accompanied by free radical production (see below). These inflammatory responses can cause significant (additional) injury through the phagocytic actions of macrophages, synthesis of toxic products, and further stimulation of immune reactions in a vicious cycle. Thus it can be argued that, at least in the initial stages of acute brain injury, a hyperinflammatory state exists. As explained in some more detail below hypothermia attenuates this proinflammatory state, but this in itself does not constitute immune suppression. On the other hand, it should be realized that the proinflammatory response outlined above is to some extent physiological; there is evidence suggesting that some inflammatory mediators have neuroprotective properties, although many others are neurotoxic [6-9]. Thus attenuating the response could have protective effects, but also some detrimental ones $[2,8,9]$.

On balance there is persuasive evidence suggesting that the production of cytokines and leukocyte infiltration is disproportionate and harmful, and can significantly increase the risk and extent of brain cell injury and infarction [6-15]. Especially, the IL-1 family appears to be important in this regard [15]. The destructive aspects of inflammation appear to outweigh the potential benefits especially in the later stages of injury [2,6-9]. Thus there is a potential time window for therapeutic interventions to block or mitigate this process before it causes permanent injury.

Many animal experiments and in vitro studies have shown that mild hypothermia can suppress harmful inflammatory reactions that damage potentially viable nerve cells and astrocytes [10-12]. Hypothermia can also decrease production of leukotrienes and nitric oxide, prevent reperfusionrelated DNA injury and lipid peroxidation, and impair neutrophil and macrophage function $[2,3]$. Thus on balance the protective effects of cooling are likely to outweigh the potential negative effects. However, this does not mean that there are no negative effects, especially if the temperature drops below $32^{\circ} \mathrm{C}$. It should be kept in mind that the suppression of inflammatory responses will occur in all organs, not just the injured ones; this is one of the reasons why inhibition of the immune response can lead to increased infection risk. Moreover, the effect is present regardless of whether a local or general hyperinflammatory state exists.

The systemic effect on the immune system can be enhanced by a decrease in the white blood cell count, which can begin at temperatures below 32 to $33^{\circ} \mathrm{C}$ (although major decreases usually occur only at temperatures below 30 to $31^{\circ} \mathrm{C}$ ). Biggar and coworkers reported a drop in WBC count from $6.0 \pm 0.6$ to $2.3 \pm 0.3$ when hypothermia of $29^{\circ} \mathrm{C}$ was induced in pigs [16]. They did 
not observe significant changes in the number of circulating mature or immature neutrophils, and reported that neutrophil demargination after administration of intravenous catecholamines was similar at $37^{\circ} \mathrm{C}$ and $29^{\circ} \mathrm{C}$. However, they also observed that administration of corticosteroids and, importantly, of endotoxins failed to stimulate the normal release of neutrophils from the bone marrow [16]. Failure of this response could significantly impact infection risks [2].

Most of these observations were made in animal experiments, but the hypothermia-induced suppression of the hyperinflammatory responses and decrease in WBC count have been confirmed in clinical reports in humans with traumatic brain injury $[10,11,17]$.

Thus the very mechanisms that provide tissue protection could simultaneously impair the patients' ability to fight infections. In addition, lowering body temperature can lead to a decrease in insulin secretion and to induction of insulin resistance [2,3]. This can lead to hyperglycaemia, which can in turn impair leucocyte function and further increase infection risks. Finally, hypothermia can cause vasoconstriction in the skin, which could increase the risk for bedsores and surgical wound infections.

In summary, based on the in vitro evidence we would expect hypothermia to inhibit the mostly harmful neuroinflammatory response and ameliorate the hyperinflammatory state that occurs after acute injury, but at the price of increasing infection risk.

Clinical evidence: Clinical studies reporting the infection risks associated with therapeutic cooling in different categories of patients with acute brain injury have produced divergent results; studies in patients who develop accidental hypothermia have mostly reported higher infection risks.

The link between accidental hypothermia in the perioperative setting and a higher incidence of surgical wound infections was first demonstrated by Kurz and coworkers in 1996 [18], and has since been confirmed in numerous studies in various categories of surgical patients [19-26]. The most recent example is a study by Seamon and coworkers, who found that intraoperative hypothermia (below $35^{\circ} \mathrm{C}$ ) was independently associated with surgical site infection rates after trauma laparotomy [26]. Local factors such as hypothermia-induced vasoconstriction in the skin may add to the underlying immunosuppressive effect to further increase the rate of wound infections. Recently, Laupland and colleagues reported that severe hypothermia $\left(<32^{\circ} \mathrm{C}\right.$, but not 32 to $\left.35.9^{\circ} \mathrm{C}\right)$ was associated with significant increases in risk for infections acquired in ICU [27].

The link between hypothermia and infections is far less clear when mild hypothermia is induced under controlled circumstances. Numerous studies in patients with post-hypoxic brain injury following cardiac arrest have not reported significant increases in rates of infections, although some have reported trends in that direction [28]. Seven multicentered trials in newborn babies treated with neonatal asphyxia for 48 to 72 hours also did not report consistent increases in infection risks [28].

In contrast, clinical studies in patients with ischemic stroke and TBI have tended to find higher risks of infection, especially pneumonia, in patients treated with hypothermia. For example, Hemmen and coworkers reported a rate of pneumonia of $50 \%$ in patients with ischemic stroke treated with hypothermia and thrombolysis, compared to $10 \%$ in controls [29]. Although the overall outcome was better in hypothermia patients in spite of the high infection rate, this indicates that use of hypothermia in these patients may present significant difficulties.

Some studies using hypothermia in patients with severe traumatic brain injury have also reported high infection rates [28]. There is evidence that this can be prevented by a combination of preventive measures, perhaps including use of antibiotic prophylaxis such as selective decontamination of the digestive tract (SDD) [28,30,31].

In one example, Kamps and coworkers reported on their use of prolonged therapeutic cooling to control intracranial pressure in patients with severe traumatic brain injury, in a setting where SDD was routinely used, and reported that infection rates were $20 \%$ in patients treated with hypothermia and $34.4 \%$ for matched controls [31]. Most notably, the risk of ventilatorassociated pneumonia was the same in patients treated with hypothermia compared with matched controls.

Conclusion: Hypothermia impairs immune function and inhibits various inflammatory responses. This is inherent to the treatment, and impairment of harmful inflammatory reactions in the brain may be one of the mechanisms through which hypothermia can exert protective effects. Hypothermia-induced insulin resistance and hyperglycemia may further increase infection risks. In clinical studies, hypothermia has been most clearly linked to infection risk in the context of accidental hypothermia; controlled therapeutic cooling appears to carry a lower risk, especially if hypothermia is used for limited periods of time ( $<48$ hours). The risk appears to increase with prolonged use, and careful monitoring is required in these patients. Prophylactic antibiotics may be considered in high-risk patients who are cooled for prolonged periods.

\section{References}

1. Heikkinen T, Järvinen A: The common cold. Lancet 2003, 361:51-59.

2. Polderman $\mathrm{KH}$ : Mechanisms of action, physiological effects, and complications of hypothermia. Crit Care Med 2009, 37:S186-S202.

3. Polderman KH, Herold I: Therapeutic hypothermia and controlled normothermia in the intensive care unit: practical considerations, side effects, and cooling methods. Crit Care Med 2009, 37:1101-1120.

4. Douglas RG Jr, Lindgren KM, Couch RB: Exposure to cold environment and rhinovirus common cold: failure to demonstrate effect. $N$ Engl J Med 1968, 279:742-747.

5. Small DL, Morley P, Buchan AM: Biology of ischemic cerebral cell death. Prog Cardiovasc Dis 1999, 42:185-207.

6. Schmidt OI, Heyde CE, Ertel W, Stahel PF: Closed head injury - an inflammatory disease? Brain Res Brain Res Rev 2005, 48:388-399.

7. Asensio VC, Campbell IL: Chemokines in the CNS: plurifunctional mediators in diverse states. Trends Neurosci 1999, 22:504-512.

8. Merrill JE, Benveniste EN: Cytokines in inflammatory brain lesions: helpful and harmful. Trends Neurosci 1996, 19:331-338.

9. Morganti-Kossmann MC, Rancan M, Stahel PF, Kossmann T: Inflammatory response in acute traumatic brain injury: a double-edged sword. Curr Opin Crit Care 2002, 8:101-105.

10. Aibiki M, Maekawa S, Ogura S, Kinoshita Y, Kawai N, Yokono S: Effect of moderate hypothermia on systemic and internal jugular plasma IL-6 levels after traumatic brain injury in humans. J Neurotrauma 1999, 16:225-232.

11. Kimura A, Sakurada S, Ohkuni H, Todome Y, Kurata K: Moderate hypothermia delays proinflammatory cytokine production of human peripheral blood mononuclear cells. Crit Care Med 2002, 30:1499-1502.

12. Suehiro E, Fujisawa H, Akimura T, Ishihara H, Kajiwara K, Kato S, Fujii M, Yamashita S, Maekawa T, Suzuki M: Increased matrix metalloproteinase-9 in blood in association with activation of interleukin- 6 after traumatic brain injury: influence of hypothermic therapy. J Neurotrauma 2004, 21:1706-1711.

13. Dietrich WD, Chatzipanteli K, Vitarbo E, Wada K, Kinoshita K: The role of inflammatory processes in the pathophysiology and treatment of brain and spinal cord trauma. Acta Neurochir Suppl 2004, 89:69-74.

14. Schoettle RJ, Kochanek PM, Magargee MJ, Uhl MW, Nemoto EM: Early polymorphonuclear leukocyte accumulation correlates with the development of posttraumatic cerebral edema in rats. J Neurotrauma 1990, 7:207-217.

15. Patel HC, Boutin $H$, Allan SM: Interleukin-1 in the brain: mechanisms of action in acute neurodegeneration. Ann NY Acad Sci 2003, 992:39-47.

16. Biggar WD, Bohn D, Kent G: Neutrophil circulation and release from bone marrow during hypothermia. Infect Immun 1983, 40:708-712.

17. Ishikawa K, Tanaka H, Shiozaki T, Takaoka M, Ogura H, Kishi M, Shimazu T, Sugimoto H: Characteristics of infection and leukocyte count in severely head-injured patients treated with mild hypothermia. J Trauma 2000, 49:912-922.

18. Kurz A, Sessler DI, Lenhardt R, Study of Wound Infection and Temperature Group: Perioperative normothermia to reduce the incidence of surgicalwound infection and shorten hospitalization. N Engl J Med 1996, 334:1209-1215.

19. McAnally HB, Cutter GR, Ruttenber AJ, Clarke D, Todd JK: Hypothermia as a risk factor for pediatric cardiothoracic surgical site infection. Pediatr Infect Dis J 2001, 20:459-462.

20. Melling AC, Ali B, Scott EM, Leaper DJ: Effects of preoperative warming on the incidence of would infection after clean surgery: a randomized controlled trial. Lancet 2001, 358:876-880.

21. Flores-Maldonado A, Medina-Escobedo CE, Ríos-Rodríguez HM, FernándezDomínguez R: Mild perioperative hypothermia and risk of wound infection. Arch Med Res 2001, 32:227-231.

22. Hedrick TL, Heckman JA, Smith RL, Sawyer RG, Friel CM, Foley EF: Efficacy of protocol implementation on incidence of wound infection in colorectal operations. J Am Coll Surg 2007, 205:432-438.

23. Wong PF, Kumar S, Bohra A, Whetter D, Leaper DJ: Randomized clinical trial of perioperative systemic warming in major elective abdominal surgery. $\mathrm{Br}$ I Surg 2007, 94:421-426. 
24. Moretti B, Larocca AM, Napoli C, Martinelli D, Paolillo L, Cassano M, Notarnicola A, Moretti $L$, Pesce $V$ : Active warming systems to maintain perioperative normothermia in hip replacement surgery: a therapeutic aid or a vector of infection? J Hosp Infect 2009, 73:58-63.

25. Sumer BD, Myers LL, Leach J, Truelson JM: Correlation between intraoperative hypothermia and perioperative morbidity in patients with head and neck cancer. Arch Otolaryngol Head Neck Surg 2009, 135:682-686.

26. Seamon MJ, Wobb J, Gaughan JP, Kulp H, Kamel I, Dempsey DT: The effects of intraoperative hypothermia on surgical site infection: an analysis of 524 trauma laparotomies. Ann Surg 2012 in press, doi: 10.1097/ SLA.0b013e31824b7e35.

27. Laupland KB, Zahar JR, Adrie C, Minet C, Vésin A, Goldgran-Toledano D, Azoulay E, Garrouste-Orgeas M, Cohen Y, Schwebel C, Jamali S, Darmon M, Dumenil AS, Kallel H, Souweine B, Timsit JF: Severe hypothermia increases the risk for intensive care unit-acquired infection. Clin Infect Dis 2012 in press, doi: 10.1093/cid/cir1033.

28. Polderman $\mathrm{KH}$ : Induced hypothermia and fever control for prevention and treatment of neurological injuries. Lancet 2008, 371:1955-1969.

29. Hemmen TM, Raman R, Guluma KZ, Meyer BC, Gomes JA, Cruz-Flores S, Wijman CA, Rapp KS, Grotta JC, Lyden PD, ICTUS-L Investigators: Intravenous thrombolysis plus hypothermia for acute treatment of ischemic stroke (ICTuS-L): final results. Stroke 2010, 41:2265-2270.

30. Polderman KH, Tjong Tjin Joe R, Peerdeman SM, Girbes AR: Effects of therapeutic hypothermia on intracranial pressure and outcome in patients with severe head injury. Intensive Care Med 2002, 28:1563-1573.

31. Kamps M, Bisschops LA, van der Hoeven JG, Hoedemaekers CW: Hypothermia does not increase the risk of infection: a case control study. Crit Care 2011, 15:R48.

\section{A9}

\section{Shivering: scores and protocols}

Neeraj Badjatia

Neurological Institute of New York, Columbia University College of Physicians and Surgeons, New York, USA

Critical Care 2012, 16(Suppl 2):A9

Shivering is both an anticipated consequence and, potentially, a major adverse effect of therapeutic hypothermia. Even mild hypothermia can elicit a vigorous thermoregulatory defense to maintain body temperature at the hypothalamic set point. In healthy humans, peripheral vasoconstriction is triggered at $36.5^{\circ} \mathrm{C}$ and shivering at $35.5^{\circ} \mathrm{C}$. Temperature thresholds for vasoconstriction and shivering are often higher than normal in brain-injured patients; therefore, these thermoregulatory defenses may occur more vigorously and at higher temperatures in these individuals. Control of shivering is essential for effective cooling, as shivering fights the cooling process, makes attaining target temperature difficult, is extremely uncomfortable, and can trigger massive increases in systemic and cerebral energy consumption and metabolic demand. The first step in treatment is adequate tools to recognize shivering. The Bedside Shivering Assessment Scale is a simple, validated four-point scale that enables repeated quantification of shivering at the bedside. Therapy for shivering should ideally stop or suppress the central thermoregulatory reflex rather than just uncoupling this response from skeletal muscle contraction, as the latter approach does not mitigate the ongoing cerebral and systemic stress response. Analgo-sedation with opioids, $\alpha_{2}$-receptor agonists, or propofol is almost always effective as a last resort to prevent shivering. However, nonpharmacological strategies as first-line interventions for shivering minimize the risk of excessive sedation, which can make neurological examination difficult and increase the risk of complications. The Columbia Anti Shivering protocol has been developed with these strategies in mind, and we base our approach on prospectively collected cooling data on 213 patients who underwent 1,388 patient-days of temperature modulation. Eighty-nine patients underwent hypothermia and 124 patients underwent induced normothermia. In $18 \%$ of patients and $33 \%$ of the total patientdays, only none-sedating baseline interventions were needed. The first agent used was most commonly dexmeditomidine half the time, followed by opiates and increased doses of propofol. Younger patients, men, and lower body surface area were factors associated with increased number of anti-shivering interventions. As noted by this protocol, a significant proportion of patients undergoing temperature modulation can be effectively treated for shivering without oversedation and paralysis.
Patients at higher risk for needing more interventions are younger men with decreased body surface area.

\section{References}

1. Choi HA, Badjatia N, Mayer SA: Hypothermia for acute brain injurymechanisms and practical aspects. Nat Rev Neurol 2012 in press, doi: 10.1038/nrneurol.2012.21

2. Choi HA, Ko SB, Presciutti M, Fernandez L, Carpenter AM, Lesch C, Gilmore E, Malhotra R, Mayer SA, Lee K, Claassen J, Schmidt JM, Badjatia N: Prevention of shivering during therapeutic temperature modulation: the Columbia anti-shivering protocol. Neurocrit Care 2011, 14:389-394.

3. Badjatia N: Hyperthermia and fever control in brain injury [review]. Crit Care Med 2009, 37(7 Suppl):S250-S257.

4. Badjatia N, Strongilis E, Prescutti M, Fernandez L, Fernandez A, Buitrago M, Schmidt JM, Mayer SA: Metabolic benefits of surface counter warming during therapeutic temperature modulation. Crit Care Med 2009, 37:1893-1897.

5. Badjatia N, Strongilis E, Gordon E, Prescutti M, Fernandez L, Fernandez A, Buitrago M, Schmidt JM, Ostapkovich ND, Mayer SA: Metabolic impact of shivering during therapeutic temperature modulation: the Bedside Shivering Assessment Scale. Stroke 2008, 39:3242-3247.

6. Badjatia N, Kowalski RG, Schmidt JM, Voorhees ME, Claassen J, Ostapkovich ND, Presciutti M, Connolly ES, Palestrant D, Parra A, Mayer SA: Predictors and clinical implications of shivering during therapeutic normothermia. Neurocrit Care 2007, 6:186-191.

A10

Controlled prophylactic normothermia

Gregor Broessner*, Marlene Fischer, Bettina Pfausler, Erich Schmutzhard Neurologic Intensive Care Unit, Medical University Innsbruck, Austria Critical Care 2012, 16(Suppl 2):A10

Introduction: Fever is a very frequent complication of intensive care treatment and an independent predictor of unfavourable outcome and mortality in most patients with an acute severe neurologic injury. Today not only treatment but even more prevention of fever has become the focus of intensivists.

Preliminary animal data for the beneficial neuroprotective effect of therapeutic hypothermia could not satisfyingly be reproduced in patients raising questions about the possible side effects of hypothermia. Controlled prophylactic normothermia $\left(36.5^{\circ} \mathrm{C}\right)$ prevents secondary injury through consequent treatment of fever and limits dose-dependent side effects through therapeutic hypothermia. Novel endovascular and gel-pad surface cooling measures have shown to be feasible and efficacious in inducing and maintaining even long-term controlled normothermia.

Rationale for treatment of fever: Fever is one of the most frequent complications of intensive care treatment. Up to $90 \%$ of patients develop at least one febrile episode within 7 days after being admitted to an ICU [1]. First of all, fever has always to be interpreted as a sign of an infection. Thus temperature modulation by any means has to include a strict protocol identifying any source of infection followed by a straightforward treatment approach. There is widespread consensus that fever alone is associated with unfavourable outcome. This consensus is a result of animal and human data over the past decades. In a meta-analysis conducted by Greer and colleagues including more than 14,000 patients, fever alone was a significant and independent predictor of morbidity and mortality across such different diseases entities as ischaemic stroke, haemorrhagic stroke and traumatic brain injury [2]. Increasing evidence from animal and human studies suggests that fever, irrespective of its cause, can directly and adversely affect neurological outcome in various types of neurological injury [3].

The pathophysiological mechanisms by which fever affects patient outcome are discussed, controversially comprising increase of metabolic demand (under circumstances of reduced supply), production of free radicals, local thermopooling, disruption of the blood-brain barrier, intracranial pressure (ICP) elevation, increased enzymatic inhibition of protein kinases, and worsened cytoskeletal proteolysis $[1,3]$.

Concept of controlled prophylactic normothermia: The aggressive treatment of fever in any patient with a severe acute neurologic injury has become increasingly important and is now the focus of many prospective studies including such patients. Whether the reduction of hyperthermia alone or even controlled hypothermia should be the treatment goal is still 
under debate and there are pros and cons for either approach. The enthusiastic preliminary results from animal hypothermia studies could not be satisfyingly reproduced when implemented in human controlled trials, shifting the focus on the possible side effects of hypothermia. Today there is a whole body of evidence that the potentially neuroprotective effects of hypothermia can be significantly diminished by its inherent side effects. It could be shown that hypothermia may lead to increased rate of infections, hypotension, shivering, disturbances in blood clotting, rewarming injuries and significant changes in pharmacokinetics and pharmacodynamics possibly limiting outcome effects of the treated patients [4-8]. Aggressive treatment of fever in the ICU without risk elevation through the side effects of therapeutic hypothermia led to the concept of controlled prophylactic normothermia. This concept is based upon strict control of body core temperature with a target of $36.5^{\circ} \mathrm{C}$ beginning as early as possible with the goal of complete fever prevention. Prophylactic controlled normothermia can therefore not be compared with the normothermia control group of most randomised trials since this novel approach aims to control temperature prophylactically and is therefore not only treatment of fever.

Experiences from clinical trials: Controlled prophylactic normothermia cannot be achieved through conventional temperature control measures including NSAIDS and conventional cooling blankets $[5,9]$. In a controlled trial conducted by our study group, reduction of fever burden (that is, body core temperature $>37.9^{\circ} \mathrm{C}$ ) was significantly higher in the endovascular cooling group than in the control group although strictly following a predefined fever management protocol including NSAIDS, opioids and conventional cooling blankets in patients with severe cerebrovascular diseases [5]. In this trial an endovascular cooling catheter was placed in the subclavian vein and prophylactic normothermia was maintained over 168 hours in patients with ischaemic stroke and intracerebral haemorrhage and over 336 hours in patients with spontaneous subarachnoid haemorrhage[5]. Safety evaluation revealed no relevant increase in direct device-related adverse events in the endovascular group.

Although there was significant decrease of the fever burden in the device group no difference could be found in the long-term, 6-month, patient follow-up. This lack of outcome efficacy may be attributed due to the increased rate of infectious complications in the device group again pointing out that state-of-the-art temperature modulation has to be combined with a standardised surveillance of infections [6].

In patients with severe traumatic brain injury $(\mathrm{GCS} \leq 8)$ direct measurement of brain temperature together with ICP is possible [10]. Since fever may deteriorate elevated ICP, prophylactic controlled normothermia should be evaluated in this patient population. In a small pilot trial, brain temperature under endovascular cooling showed that even under normothermia $\left(36.5^{\circ} \mathrm{C}\right)$ brain temperature almost reaches body core temperature [11]. This is of utmost interest as brain temperature exceeds body core temperature even under physiological circumstances and even more under fever with a peak gap $>2^{\circ} \mathrm{C}[10]$. As the brain is the target tissue of all neuroprotectants, it is now evident that even controlled endovascular normothermia can significantly lower brain temperature in TBI patients.

Others could achieve normothermia through a novel surface cooling device using gel-coated energy transfer pads, directly applied to the skin in patients with spontaneous subarachnoid haemorrhage and severe traumatic brain injury $[9,12]$.

Questions to be addressed in future trials: What is the optimal target temperature and duration for temperature modulation in various disease entities? What is the appropriate approach to inherent complications such as shivering, infections, pharmacodynamic and pharmacokinetic disturbances? Are surface and endovascular treatment approaches equivalent?

Conclusion: Controlled prophylactic normothermia is not only fever reduction but aims to strictly maintain the body temperature at $36.5^{\circ} \mathrm{C}$. Induced as early as possible the duration should cover the acute phase of the neurological injury, minimising secondary additional neurological impairment though prevention of fever. Endovascular and surface cooling measures using gel-coated pads have shown to be efficacious and feasible in inducing and maintaining normothermia whereas conventional temperature control measures including NSAIDs and conventional cooling blankets are not sufficient to prevent fever. Side effects known from therapeutic hypothermia such as shivering and increased rate of infections also may occur under controlled prophylactic normothermia, although to a lesser extent. Nevertheless, a consequent prevention of those limiting factors should be kept in mind when applying controlled prophylactic normothermia.

References

1. Marion DW: Controlled normothermia in neurologic intensive care. Crit Care Med 2004, 32(Suppl):S43-S45.

2. Greer DM, Funk SE, Reaven NL, et al: Impact of fever on outcome in patients with stroke and neurologic injury: a comprehensive metaanalysis. Stroke 2008, 39:3029-3035.

3. Polderman KH: Induced hypothermia and fever control for prevention and treatment of neurological injuries. Lancet 2008, 371:1955-1969.

4. van den Broek MP, Groenendaal F, Egberts AC, et al: Effects of hypothermia on pharmacokinetics and pharmacodynamics: a systematic review of preclinical and clinical studies. Clin Pharmacokinet 2010, 49:277-294.

5. Broessner G, Beer R, Lackner P, et al: Prophylactic, endovascularly based, long-term normothermia in ICU patients with severe cerebrovascular disease: bicenter prospective, randomized trial. Stroke 2009, 40:e657-e665.

6. Broessner $G$, Lackner P, Fischer $M$, et al: Influence of prophylactic, endovascularly based normothermia on inflammation in patients with severe cerebrovascular disease: a prospective, randomized trial. Stroke 2010, 41:2969-2972.

7. Clifton GL, Miller ER, Choi SC, et al: Lack of effect of induction of hypothermia after acute brain injury. N Engl J Med 2001, 344:556-563.

8. Clifton GL, Valadka A, Zygun D, et al: Very early hypothermia induction in patients with severe brain injury (the National Acute Brain Injury Study: Hypothermia II): a randomised trial. Lancet Neurol 2011, 10:131-139.

9. Oddo M, Frangos S, Milby A, et al: Induced normothermia attenuates cerebral metabolic distress in patients with aneurysmal subarachnoid hemorrhage and refractory fever. Stroke 2009, 40:1913-1916.

10. Rumana $\mathrm{CS}$, Gopinath SP, Uzura M, et al: Brain temperature exceeds systemic temperature in head-injured patients. Crit Care Med 1998, 26:562-567.

11. Fischer M, Dietmann A, Lackner $P$, et al: Endovascular cooling and endothelial activation in hemorrhagic stroke patients. Neurocrit Care 2011 in press.

12. Oddo M, Frangos S, Maloney-Wilensky $E$, et al: Effect of shivering on brain tissue oxygenation during induced normothermia in patients with severe brain injury. Neurocrit Care 2010, 12:10-16.

\section{COOLING IN NEUROLOGY AND NEUROSURGERY}

A11

Therapeutic hypothermia in traumatic brain injury

Jonathan KJ Rhodes

University of Edinburgh, Department of Anaesthesia, Critical Care and Pain Medicine, Intensive Care Unit, Western General Hospital, Edinburgh, UK Critical Care 2012, 16(Suppl 2):A11

Hypothermia has profound effects on the brain function but importantly is potentially protective against both focal and global injuries. Aspects of the biochemical response to acute ischaemia and trauma, which are associated with poor outcome, can be inhibited by cooling. Unlike many pharmacological treatments that tend to antagonise a single neurochemical process, hypothermia offers a simple method of inhibiting multiple pathological processes simultaneously. It therefore has the potential, if applied correctly, to improve outcomes after acute brain injuries, where drug trials have so far failed.

The systemic cooling of patients after acute brain injury is an established treatment modality in many neuro-ICUs. It is a strategy for protecting the injured brain that makes intuitive sense and can reduce both intracranial pressure and the potential for ischaemic secondary insults. Basic science evidence also suggests that cooling can attenuate many secondary biochemical cascades that are activated after acute injury.

However, despite these multiple lines of supportive evidence there is as yet no confirmation from a high-quality randomised controlled trial that prophylactic hypothermia improves outcome or reduces mortality.

This talk will look at the potentially beneficial effects of hypothermia on the biochemistry of acute brain injury, consider the reasons for the failure to demonstrate clinical efficacy and review the supportive data from 
meta-analysis, suggesting how hypothermia might be best delivered. Finally I will discuss EuroTherm3235, a European Society of Intensive Care Medicine funded multicentre randomised controlled trial investigating prophylactic hypothermia in traumatic brain injury, which draws on the lessons from the available literature.

\section{References}

1. Clifton GL, Valadka A, Zygun D, et al: Very early hypothermia induction in patients with severe brain injury (the National Acute Brain Injury Study: Hypothermia II): a randomised trial. Lancet Neurol 2011, 10:131-139.

2. Andrews PA, Sinclair HL, Battison CG, et al: European Society of Intensive Care Medicine study of therapeutic hypothermia $\left(32-35^{\circ} \mathrm{C}\right)$ for intracranial pressure reduction after traumatic brain injury (the Eurotherm 3235 trial). Trials 2011, 12:8.

\section{A12}

\section{Hypothermia in spinal cord injury}

Guadalupe A Castillo-Abrego $0^{1,2}$

${ }^{1}$ Neurocritical Care Unit, Caja de Seguro Social Hospital, Panamá City, Panamá; ${ }^{2}$ Medical Staff Critical Care Unit, Punta Pacifica Hospital: John Hopkins Affiliated Hospital, San Fernando Hospital, Centro Médico Paitilla, Hospital Nacional, Panamá City, Panamá

Critical Care 2012, 16(Suppl 2):A12

Historical considerations: In 1862 Edwin Smith, Egyptologist, acquired a papyrus that was thought to be between 2,500 and 3,000 years old. It was translated in 1930 by James Breasted, and was found to contain information on medical therapies performed on 48 patients, including six cases of cervical spine trauma. In the papyrus these injuries were described as 'dislocation of the vertebrae of the neck with unconscious arms and legs, where urine was drained and the phallus was erect, and it was determined that this cannot be treated' [1].

Other physicians like Hippocrates and Claudius Galen made historical reference to the management of spinal cord injury. Galen studied injuries in gladiators, and described 'that injury of the spinal cord caused paralysis and loss of sensation below the level of injury ... and determined that high spinal cord injuries are incompatible with life' [2,3].

Chaulic Guy (1300 to 1360) carried out innovations in the management of traumatic bone injuries by introducing boards and suspensions to treat these fractures; however, he gave a pessimistic view on spinal injuries $[4,5]$.

Ambrose Pare (1510 to 1590 ) developed spine surgery, and along with Hildanus Fabricius (1560 to 1634) used traction techniques, reduction and utilization of wood frames to treat cervical spine fractures [6].

Louis Pasteur's (1822 to 1895) surgical innovations and discoveries and the advent of asepsis and antisepsis by Semmelweis (1816 to 1868) and others, and primarily the application of general anesthesia by Morton (1819 to 1868) and others gave a new impulse to this field. Together these developments helped reduce surgical mortality and infections $[5,7]$.

In the nineteenth century, anatomy and physiology of the central nervous system were studied. The neuron doctrine was developed by Santiago Ramon y Cajal (1852 to 1934), who demonstrated the individuality of nerve cells and the connections that they have with each other $[8,9]$.

Alfred R Allen between 1908 and 1911 developed a reproducible and quantifiable model that allowed induction of a uniform traumatic injury to the spine, and also explained the pathophysiological changes seen in spinal trauma, including key aspects of secondary spinal cord injury. In 1972 Tarlov showed that symptoms caused by compression of the spinal cord for more than 12 hours could sometimes still be reversed. During this period 'progressive central hemorrhagic necrosis' was identified, which consists of bleeding from the gray matter of the spinal cord central necrosis and destruction of white matter, with subsequent cavitations $[10,11]$. Finally, one of the possible immunomodalities of neuroprotection was suggested in 1968 by Albin and White who applied local hypothermia with favorable results in animals [12]. However, use of hypothermia was limited due to fear of side effects [13-17].

These preliminary studies were difficult to interpret due to the limited number of patients, lack of controls, concomitant surgical procedures and the concomitant use of drugs such as methylprednisolone [18]. In recent years there has been a renewed interest in the use of moderate therapeutic hypothermia due to its demonstrated neuroprotective effects in other areas. In Panama, we have been administering therapeutic hypothermia in selected cases with severe spinal cord injury. More frequently we apply fever management through endovascular, surface pads or medical thermomodulation protocols.

Epidemiological aspects of spinal cord injury: No accurate numbers on spinal cord injury are available for Panama and Central America. In the United States there are around 12,000 new cases of traumatic spinal cord injury each year, not including those who die at the scene of the accident [19]. Approximately 1.3 million Americans have some type of chronic paralysis resulting from spinal cord injury $[20,21]$. The leading causes are motor vehicle accidents $(41.3 \%)$, falls $(27.3 \%)$, acts of violence $(15 \%)$ and recreational activities (7.9\%). Apart from the medical and personal consequences for the patient, the economic impact in terms of ability to work is significant.

Spinal cord injury, neurophysiological aspects - how can therapeutic hypothermia help?: We will provide a concise description of the pathophysiological changes that occur after a spinal injury that might justify use of therapeutic hypothermia to mitigate ongoing destructive processes. Spinal cord injury is a process that can be divided into three phases [22,23]. The primary mechanism refers to the direct mechanical damage. This may include compression, stretching, persistent concussion, contusion, compression and laceration of the spinal cord $[22,23]$. The secondary mechanism involves a cascade of events at the cellular level triggered by the primary mechanism. These biochemical events are responsible for increased tissue damage and promote the apoptotic cascade. These include those listed in Table 1. The healing mechanism (third mechanism) begins in the days after injury and can last for months or years. This can paradoxically increase the neurological damage. Healing in the primary injury takes place by neutrophils, macrophages and lymphocytes, reactive astrocytes, Schwann cells, meningeal fibroblasts, and microglia invasion. Scar formation forms a barrier for cellular and molecular axonal regeneration.

Therapeutic hypothermia has been used in various types of neurological injury such as stroke, post-anoxic encephalopathy, and traumatic brain injury. Some of these results have been extrapolated to spinal cord trauma patients.

Moderate therapeutic hypothermia has been shown to affect the apoptotic cascade as well as other destructive mechanisms, ranging from improved energy balance, reduction of mitochondrial dysfunction, decreased vascular permeability and capillary leakage, mitigation of cell membrane injury, improvement of intracellular acidosis, mitigation of DNA injury, reduction in metabolic demand, and a decrease in proinflammatory cytokine and free radical release [24-28].

It is important to realize that there is a window time, perhaps several hours to days after the injury, during which this treatment modality can influence the course of events [29].

Experimental studies and supporting evidence: $Y u$ and colleagues used therapeutic hypothermia $\left(33^{\circ} \mathrm{C}\right)$ after 30 minutes of ischemic injury to the thoracic spinal cord in rats, and reported improvement of motor function at the microscopic level associated with cooling [30]. Others reported improvements in functional outcome in various animal models associated with cooling [30-32]. In an animal study of spinal cord ischemia, hypothermia increased the duration of ischemia required to produce neurological deficits [31].

Moderate hypothermia has also shown potential benefits in invasive procedures including aortic clamp cross-clamping during thoracic surgery $[33,34]$. This can be regarded as indirect evidence that hypothermia could protect the spinal cord from ischemic injury, although the mechanisms in traumatic injury may be different from surgical trauma and ischemia.

Fever is a common complication in patients with spinal cord injury [35-37]. Of the identifiable etiologies the most common cause is infection (especially pneumonia and urinary tract infections). However, fever of unknown origin is the most frequent diagnosis, occurring in $66 \%$ of patients. It should be realized that patients with spinal cord injury have a high incidence of thermoregulatory problems, which can contribute to the high incidence of FUO $[38,39]$.

Of note, $\mathrm{Yu}$ and colleagues demonstrated in an experimental study in rats that post-traumatic hyperthermia in thoracic spinal cord injury worsened behavioral and histopathological damage compared with normothermia, and was associated with an increase of overall contusion volume by increasing the vulnerability of both gray and white matter structures compared with normothermia [40].

Role of moderate hypothermia in the clinical scenario of patients with spinal cord injury: Early clinical studies with irrigation to induce 


\section{Table 1(Abstract A12)}

1. After the death of an oligodendrocyte, a progressive Walerian degeneration takes place from the site of primary injury, compromising the integrity of axons and mitochondria. Simultaneously caspase activation and apoptosis occur, leading finally to demyelination and release of nerve growth factor.

2. Vascular ischemia, impaired autoregulation, neurogenic shock, radial and axial hemorrhage, compromised microcirculation, collapsed veins, venous obstruction, vasospasm and thrombosis.

3. Ionic alterations: intracellular and extracellular calcium overload, increase of sodium and potassium.

4. Accumulation of neurotransmitters such as dopamine, norepinephrine, serotonin and glutamate at the extracellular level.

5. Release of arachidonic acid, production of free radicals and lipid eicosanoid peroxidation.

6. Endogenous opioid activation

7. Formation of cytotoxic and vasogenic edema

8. (Hyper)inflammatory response

9. Failure of ATP-dependent intracellular processes

local hypothermia produced conflicting results [41]. However, the advent of more reliable cooling devices that can better maintain body temperature within a predetermined range have improved our ability to deliver targeted therapy.

Hypothermia has been used to protect the spinal cord and prevent paraplegia during high aortic cross surgery. In one study with long-term follow-up, the incidence of spinal cord injury in patients undergoing highrisk thoracoabdominal aneurysm repair under hypothermia over a 10-year period the risk of $\mathrm{SCl}$ was $18 \%$ in hypothermic patients compared with $29 \%$ in historical controls $(P<0.01)[29,33]$.

Interest in hypothermia for spinal cord injury received a boost when in 2007 a high-profile case was reported. An NFL player suffered a complete AIS A cervical spine injury, and was treated immediately on the football field with moderate hypothermia. This individual had a much better than expected outcome and this led to a spate of publications on the potential use of hypothermia for spinal cord injury [40,42-44]. Of course, such anecdotal evidence cannot prove the benefit of any therapy, and professional organizations such as the American Association of neurological surgeons, Neurological and Spine Surgery Joint Sections and Joint Section of Trauma concluded that there was currently not enough evidence available to recommend or discourage the practice of therapeutic hypothermia as a treatment for spinal cord injury [42,45-47].

In 2009 Levi and colleagues successfully tested the safety and feasibility of systemic hypothermia induction in spinal cord injury with an endovascular cooling device [48]. The authors treated 14 patients with AIS A spinal cord injury with moderate hypothermia. At a median follow-up of 1 year they found an improved conversion rate: in 6/14 cooled patients there was an improvement of the neurological examination (three patients improved to AIS B, two patients improved to AIS C and one patient improved to AIS D). This represents an improvement rate of $42.8 \%$, higher than the 12.5 to $20 \%$ found in various other studies where patients had not been treated with hypothermia $[49,50]$. Complications associated with cooling were mostly respiratory issues (atelectasis and pneumonia) but these rates were similar in the other studies where cooling had not been used. Adverse events such as coagulopathy, deep venous thrombosis and pulmonary embolism were not reported in the patients treated with hypothermia. This is the first investigation on the safety systemic cooling in acute spinal cord injury [48,51]. To determine efficacy will require randomized controlled clinical studies. Currently there are plans to organize such a trial, which will involve 17 centers to determine whether moderate hypothermia improves outcome in a larger population of patients with acute spinal cord injury. Details can be found at online: http://www.miamiproject.org. The protocol calls for induction of hypothermia within 6 hours of injury, to be maintained by endovascular cooling, where they will evaluate the safety of different durations of hypothermia, outcomes and risks [47].

Fever in patients with spinal cord injury, if not controlled promptly, may lead to increased morbidity and mortality because of hyperthermic damage to cells. Therefore controlling fever is an important goal of care in these patients. Preliminary data suggest that endovascular cooling can be used effectively for this purpose [52,53].

There are no proven treatments with high grades of scientific evidence for the devastating consequences of spinal cord injury. In Panama, selected patients with ASIA A lesions are treated with therapeutic cooling for a period of 24 to 48 hours. This can be done in either mechanically ventilated or nonventilated patients. Other aspects of treatment include keeping adequate medullar perfusion pressure, normothermia throughout their ICU stay (accomplished by pharmacological interventions, mechanical cooling either with a surface cooling or endovascular device), early enteral immunonutrition, and various tests such as somatosensory evoked potentials.

Conclusion: So far there are no proven therapeutic interventions that improve outcome in severe spinal cord injury. Hypothermia appears to be a promising treatment in this population, and needs to be studied in prospective clinical trials. Fever control should be a goal of care in these patients.

Acknowledgements: The author thanks Dr Kees Polderman, Dr Guillermo Castillo, Dr Waltter Kravcio, Dr Javier Alvarado and Franklin Paulino for their support.

References

1. Wilkins RH: Neurosurgical classic XVII. Edwin Smith surgical papyrus. J Neurosurg 1964, 24:240-244.

2. Marketos SG, Skiadas PK: Hippocrates. The father of spine surgery. Spine 1999, 24:1381-1387.

3. Marketos SG, Skiadas PK: Galen: a pioneer of spine research. Spine 1999, 24:2358-2362.

4. Goodrich JT: History of spine surgery in the ancient and medieval worlds. Neurosurg Focus 2004, 16:1-3.

5. Lifshutz J, Colohan A: A brief history of therapy for traumatic spinal cord injury. Neurosurg Focus 2004, 16:1-8.

6. Sontang VKH: History of degenerative and traumatic disease of the spine. A History of Neurosurgery in its Scientific and Professional Contexts Parke Ridge, IL: American Association of Neurological Surgeons: Greenblatt SH 1997, 355-371.

7. Knoeller SM, Seifried C: Historical perspective. History of spinal surgery. Spine 2000, 25:2838-2843.

8. McHenry LC: Garrison's History of Neurology Springfield, IL: Charles C. Thomas 1960, 265-424.

9. Naderi S, Ture U, Pait TG: History of spinal cord localization. Neurosurg Focus 2004, 16:1-6.

10. Collins WF: Historical perspective on spinal cord injury. Neurotrauma New York: McGraw Hill: Narayan RK, Wilberger JE, Povlishock JT 1995, 1041-1047.

11. Tarlov I: Acute spinal cord compression paralysis. J Neurosurg 1972, 36:10-20.

12. Albin MD, White RJ, Acosta Rua G, et al: Study of functional recovery produced by delayed localized cooling after spinal cord injury in primates. J Neurosurg 1968, 29:113-120.

13. Bricolo A, Ore GD, Da Pian R, et al: Local cooling in spinal cord injury. Surg Neurol 1976, 6:01-106.

14. Demian YK, White RJ, Yashon D, et al: Anesthesia for laminectory and localized cord cooling in acute cervical spine injury. Report of three cases. Br J Anaesth 1971, 43:973-979.

15. Koons D, Gildenberg PL, Dohn DF, et al: Local hypothermia in the treatment of spinal cord injuries. Report of seven cases. Clev Clin Q 1972, 39:109-117.

16. Selker RG: Icewater irrigation of the spinal cord CH. Surg Forum 1971, 22:411-413. 
17. Tator $\mathrm{CH}$, Deecke $\mathrm{L}$ : Value of normothermic perfusion, hypothermic perfusion and durotomy in the treatment of experimental acute spinal cord trauma. J Neurosurg 1973, 39:52-64.

18. Hansebout RR, Kuchner EF: Effect of local hypothermia and of steroids upon recovery from experimental spinal cord injuries compression injury. Surg Neurol 1975, 4:531-536.

19. Spinal cord injury, facts and figures at a glance. J Spinal Cord Med 2008, 31:357-358.

20. Paralysis and Spinal Cord Injury in the United States Short Hills, NJ: Christopher and Dana Reeve Foundation 2009.

21. Spinal cord injury facts and figures at a glance. J Spinal Cord Med 2010, 33:439-440.

22. Hagg T, Oudega M: Degenerative and spontaneous regenerative process after spinal cord injury. J Neurotrauma 2006, 23:248-261.

23. Mc Donald J, Belegu V: De myelization and remyelination after spinal cord injury. J Neurotrauma 2006, 23:345-359.

24. Polderman $\mathrm{KH}$ : Therapeutic hypothermia in the intensive care unit: problems, pitfalls and opportunities (review). Part 1: indications and evidence. Intensive Care Med 2004, 30:556-575.

25. Mayer SA, Sessler DI: Therapeutic Hypothermia New York: Marcel Dekker, 12005.

26. Hayashi N, Dietrich DW: Brain Hypothermia Treatment Tokyo: SpringerVerlag, 12004.

27. Polderman KH: Mechanism and potential side effects of therapeutic mild hypothermia. Cardiac Arrest: The Science and Practice of Resuscitation Medicine Cambridge: Cambridge University Press: Paradis N, Halperin H, Kern K, Wenzel V, Chamberlain D , 2 2007, 859-870.

28. Edwards $P$, Arango $M$, Balica $L$, et al: CRASH trial collaborators. Final results of MRC CRASH, a randomized placebo controlled trial of intravenous corticosteroid in adult with head injury - outcomes at 6 months. Lancet 2005, 365:1957-1959.

29. Polderman KH: Induced hypothermia and fever control for prevention and treatment of neurological injuries. Lancet 2008, 371:1955-1969.

30. Yu GC, Jimenez O, Marcillo AE, et al: Beneficial effects of modest systemic hypothermia on locomotors function and histopathological damage following contusion-induced spinal cord injury in rats. J Neurosurg 2000, 93:85-93.

31. Pearse DD, Lo TP Jr, Cho KS, et al: Histopathological and behavioral characterization of a novel cervical spinal cord displacement contusion injury in the rat. J Neurotrauma 2005, 22:680-702.

32. Lo TP, Cho KS, Grag MS, et al: Systemic hypothermia improves histological and functional outcome after cervical spinal cord contusion in rats. $J$ Comp Neurol 2009, 514:433-448.

33. Hartemink K, Wisselink W, Rauwerda J, Girbes A, Polderman K: Novel applications of therapeutic hypothermia: report of three cases. Crit Care 2004, 8:R343-R346.

34. Fehrenbacher JW, Hart DW, Huddleston E, et al: Optimal end organ protection for thoracic and thoracoabdominal aortic aneurysm repair using deep hypothermia circulatory arrest. Ann Thorac Surg 2007, 83:1041-1046.

35. Mc Kinley W, McNamee S, Meade M, Kandra K, et al: Incidence, etiology and risk factors for fever following acute spinal cord injury. J Spinal Cord Med 2006, 29:501-506.

36. Ulger F, Dilek A, Karakaya D, Senel A, et al: Fatal fever of unknown origin in acute cervical spinal cord injury: five cases. J Spinal Cord Med 2009, 32:343-348.

37. Scott SR, Matthew MJ, Philip MS, William LB, et al: Fatal malignant hyperpyrexia in a cervical spine-injured patient. J Trauma 2005, 58:375-377.

38. Montgomerie JZ: Infections in patients with spinal cord injuries. Clin Infect Dis 1997, 25:1285-1290.

39. Sugarman $B$, Brown $D$, Musher D: Fever and infection in spinal cord injury patients. JAMA 1982, 248:66-70.

40. Yu CG, Jagid J, Ruenes $G$, et al: Detrimental effects of systemic hyperthermia on locomotor function and histophatological outcome after traumatic spinal cord injury in the rat. Neurosurgery 2001, 49:152-159.

41. Kwon BK, Mann C, Sohn HM, et al: hypothermia for spinal cord injury. Spine 2008, 371:1955-1969.

42. Dietrich WD: Presidential address. Presented at 34th Annual Meeting of the Cervical Spine Research Society; 30 November 2006; Florida, USA.

43. Goldstein J: Lower Inf Body Temperature Shows Promise for Trauma Treatment. [http://sci-info-pages.com/2006/05/lowering-body-temp-showspromise-for.html].
44. Hartemink KJ, Wisselink W, Rauweda JA, et al: Novel applications of therapeutic hypothermia: report of three cases. Crit Care 2004, 8: R343-R346.

45. Cappuccino A, Bisson LJ, Carpenter B, et al: The use of systemic hypothermia for the treatment of an acute cervical spinal cord injury in a professional football player. Spine 2010, 35:E57-E62.

46. Dietrich DW: Therapeutic hypothermia for spinal cord injury. Crit Care Med 2009, 12:183-187.

47. Dietrich D, Cappucino A, Capuccino H: Systemic hypothermia for the treatment of acute cervical spinal cord injury in sports. Curr Sports Med Rep 2011, 10:50-54.

48. Levi A, Barth Green, Wang M, et al: Clinical application of modest hypothermia after spinal cord injury. I Neurotrauma 2009, 26:407-415.

49. Geisler FH, Coleman WP, Crieco G, Poonian D, Sygen Study Group: The Sygen multicenter acute spinal cord injury study. Spine 2001, 26(24 Suppl):S87-S98.

50. Van Middendorp JJ, Hosman AJ, Pouw MH, EM-SCI Study Group: ASIA impairment scale conversion in traumatic $\mathrm{SCl}$ : is it related with the ability to walk? A descriptive comparison with functional ambulation outcome measures in 273 patients. Spinal Cord 2009, 47:555-560.

51. Levi A, Casella G, et al: Clinical outcome using modest intravascular hypothermia after acute cervical spinal cord injury. Neurosurgery 2010, 66:670-677.

52. Marion DW: Controlled normothermia in neurologic intensive care. Crit Care Med 2004, 32:S43-S51.

53. Tripathy W, Whitehead C: Endovascular cooling for severe hyperthermia in cervical spine injury. Neurocrit Care 2011, 15:525-528.

\section{A13}

Phase 2/3 study of intravenous thrombolysis and hypothermia for acute treatment of ischemic stroke (ICTuS 2/3)

Thomas Hemmen ${ }^{1 *}$, Karen Rapp ${ }^{1}$, Rema Raman ${ }^{1}$, Mauricio Concha ${ }^{2}$, Gregor Brössner ${ }^{3}$, Erich Schmutzhard ${ }^{3}$, Gilda Tafreshi ${ }^{4}$, Vivek Misra ${ }^{5}$, Salvador Cruz-Flores ${ }^{6}$, Rainer Kollmar ${ }^{7}$, David Brown ${ }^{8}$, Irfan Altafullah' ${ }^{9}$, Patrik Michel ${ }^{10}$, Andrei Alexandrov ${ }^{11}$, Carlos Smith ${ }^{12}$, Julie Jurf ${ }^{1}$,

Mary Jane Hess ${ }^{5}$, James Grotta ${ }^{5}$, Patrick D Lyden ${ }^{13}$

${ }^{1}$ UCSD Medical Center, San Diego, CA, USA; ${ }^{2}$ Sarasota Memorial Hospital, Sarasota, FL, USA; ${ }^{3}$ University Hospital Innsbruck, Austria; ${ }^{4}$ Scripps Mercy Hospital, San Diego, CA, USA; ${ }^{5}$ University of Texas, Houston, TX, USA; ${ }^{6} \mathrm{St}$ Louis University, St Louis, MO, USA; ${ }^{7}$ University of Erlangen, Germany; ${ }^{8}$ Hoag Hospital, Newport Beach, CA, USA; ${ }^{9}$ North Memorial Hospital, Robbinsdale, MN, USA; ${ }^{10}$ University of Lausanne, Switzerland; ${ }^{11}$ University of Alabama, Birmingham, AL, USA; ${ }^{12}$ St Joseph Hospital, Tampa, FL, USA; ${ }^{13}$ Cedars Sinai Medical Center, Los Angeles, CA, USA

Critical Care 2012, 16(Suppl 2):A13

Background: The ICTuS trial showed feasibility of endovascular hypothermia for acute ischemic stroke [1]; ICTUS L confirmed safety and feasibility of endovascular hypothermia during thrombolysis [2]. The ICTuS $2 / 3$ study seeks to determine whether the combination of thrombolysis and hypothermia is superior to thrombolysis alone for the treatment of acute ischemic stroke. A phase 2 study will include 450 patients to assess the safety of various protocol changes, to demonstrate sufficient recruitment, and to allow an interim analysis for futility. If pre-specified milestones are achieved the study will be enlarged to a 1,800-patient phase 3 efficacy study.

Methods: This is a prospective, randomized, single-blind, multicenter phase $2 / 3$ study. We aim to include ischemic stroke patients treated within 3 hours of symptom onset with IV tPA (according to FDA or EMEA protocol), NIHSS $\geq 7$ and $\leq 20$, age 22 to 80 . Patients are randomly assigned to either hypothermia permissively targeted to $33^{\circ} \mathrm{C}$ or normothermia. Favorable outcome is defined as a 90-day Modified Rankin score (mRS) of 0 or 1. Secondary outcome measures are: 90-day NIHSS, Barthel Index (BI), mortality, shift analysis of the mRS, global odds ratio of $\mathrm{mRS}, \mathrm{BI}, \mathrm{NIHSS}$, incidence of symptomatic intracranial hemorrhage and 90-day Montreal Cognitive Assessment. An interim analysis for futility is planned near the end of phase 2. In addition to futility, analyses will assess the frequency of target temperature reached within 6 hours from symptom, pneumonia rate, safety profile of iced saline infusion and study-wide average enrollment rate.

Results: The study team initiated 11 study sites in the USA and two in Europe. Enrolment began in December 2010. Currently, 37 subjects are 
enrolled. A full DSMB review of experience to date allowed the study to continue enrollment. A safety review after the first 50 patients is expected in late 2012.

Conclusions: ICTuS $2 / 3$ is the largest trial of endovascular hypothermia for acute stroke currently running. There appear to be no safety or feasibility concerns.

Acknowledgements: NINDS grants P50NS5044148 (UCSD) and P50NS044227 (Houston).

References

1. Lyden PD, Allgren RL, Ng K, Akins P, Meyer B, Al-Sanani F, Lutsep H, Dobak J, Matsubara BS, Zivin J: Intravascular Cooling in the Treatment of Stroke (ICTuS): early clinical experience. J Stroke Cerebrovasc Dis 2005, 14:107-114.

2. Hemmen TM, Raman R, Guluma KZ, Meyer BC, Gomes JA, Cruz-Flores S, Wijman CA, Rapp KS, Grotta JC, Lyden PD: Intravenous thrombolysis plus hypothermia for acute treatment of ischemic stroke (ICTUS-L): final results. Stroke 2010, 41:2265-2270.

\section{A14}

Therapeutic hypothermia decreases growth of perihemorrhagic edema and prevents critical increase of intracranial pressure in large intracerebral haemorrhage

Rainer Kollmar*, Stefan Schwab, Dimitre Staykov

Department of Neurology, Friedrich-Alexander-University of Erlangen-

Nuernberg, Erlangen, Germany

Critical Care 2012, 16(Suppl 2):A14

Background: Intracerebral hemorrhage $(\mathrm{ICH})$ accounts up to $15 \%$ of all firstever strokes and is associated with high mortality, morbidity, and disability $[1,2]$. Main factors contributing to poor outcome within the first days after symptom onset are hematoma size, early hematoma growth, the presence of intraventricular hemorrhage, and the size of perihemorrhagic edema (PHE) [3]. PHE leads to secondary injury by a complex pathophysiological cascade following $\mathrm{ICH}$. Above all, mass effects of PHE can lead to critical increase of intracranial pressure and subsequent herniation. Since the volume of PHE increases within the first days after $\mathrm{ICH}$ and is correlated to $\mathrm{ICH}$ volume, PHE represents a meaningful target for interventions [3]. Therapeutic hypothermia (TH) is a promising candidate to treat or even prevent PHE. Experimental data indicate that TH is neuroprotective after acute brain injury including $\mathrm{ICH}$ and reduces PHE [4]. In a proof of concept study, we investigated the effects of mild $\mathrm{TH}$ of $35^{\circ} \mathrm{C}$ over a period of 10 days in patients suffering from large $(>25 \mathrm{ml}) \mathrm{ICH}$ and compared these patients with a historical control group [5]. Even with standard treatment, $\mathrm{ICH}$ of this size has an extremely high mortality and almost never leads to acceptable neurological outcome [2]. In our study, TH prevented the increase of PHE and led to a superb in-hospital survival rate and an acceptable long-term survival and grade of neurological deficits $[5,6]$. Because of these promising results, we established an institutional protocol in the Department of Neurology at University Hospital Erlangen and treated patients with large ICH with mild TH. Here, we report data of 20 patients with large ICH treated in our neuro-ICU.

Materials and methods: All patients with large ICH were treated by a detailed institutional protocol that is in line with our ongoing prospective study [7]. Patients aged over 18 years with primary ICH at the level of the basal ganglia or thalamus and a hematoma volume of over $25 \mathrm{ml}$ on initial CCT were treated by TH. Patients have been treated within the first 12 hours after symptom onset if they had a score on the Glasgow Coma Scale (GCS) of $\leq 8$ at presentation or early worsening by 2 points with subsequent endotracheal intubation and neurointensive care treatment. All patients received invasive ICP measurement by external ventricular drainage or a parenchymal probe. Relatives were informed about the treatment and approved this regimen. Patients have not been treated by TH if any clinical signs of herniation such as pupillomotoric defects or bilateral signs of the pyramidal tract at baseline could be observed or the treating team agreed to a do-not-resuscitate order. Laboratory exclusion criteria included an international normalized ratio $>1.5$, a thrombocyte count below $70,000 / \mu$ l or leukocytosis $>20,000 / \mu \mathrm{l}$ on admission. The presence of intraventricular hemorrhage has not been an exclusion criterion, since we have a standardized protocol, including external ventricular drainage, intraventricular clot lysis and the use of lumbar drainage. Patients who have been randomized to the control arm of our multicenter randomized controlled trial $\mathrm{CINCH}$ [7] are not reported here.

Intervention of therapeutic hypothermia: Patients were treated with an endovascular catheter-based cooling system (ICY catheter; Zoll Medical, USA) positioned in the femoral vein as described previously [5]. The target temperature has been set to $35.0^{\circ} \mathrm{C}$. The body core temperature has been measured by a urinary bladder catheter. As soon as body core temperature drops below $36.0^{\circ} \mathrm{C}$, patients are covered by a warming blanket to avoid shivering. Ten days after initiation of $\mathrm{TH}$, patients received slowly, controlled rewarming by $0.1^{\circ} \mathrm{C} /$ hour. The catheter has been changed at least once during the treatment period, preferably on day $4 \pm 0.5$ after ICH, or if clinically indicated

Outcome analysis: Patients have been analysed for in-hospital mortality, mortality and functional outcome (modified Ranking Scale and Barthel Index) on days 90 and 180 after $\mathrm{ICH}$.

Semiautomatic assessment of ICH and PHE volume: The Siemens Leonardo $\mathrm{V}$ software for semiautomatic $\mathrm{CT}$ volumetry has been used for assessment of hematoma and perihemorrhagic edema volumes. The procedure has been described in detail before [3].

Statistical analysis: Statistical tests were performed with SPSS 16.0 software package. Data are given as mean \pm standard deviation, if not indicated differently. Normality of distribution was tested using the ShapiroWilk and Kolmogorov-Smirnov tests. Absolute edema values were not distributed normally. Accordingly, single comparisons of absolute edema between the two groups at different time points were performed using the nonparametric Mann-Whitney $U$ test. All other data were distributed normally. The unpaired $t$ test was used for single comparisons of ICH values between the two groups. $P<0.05$ was considered significant.

Results: Patient characteristics: A total of 20 patients have been treated with mild hypothermia so far. There have been no significant differences between these patients and our historic control group $(n=25$; Table 1). Overall, the volume of ICH in the initial CT was large with $57 \pm 25 \mathrm{ml}$ for the hypothermia group and $59 \pm 31 \mathrm{ml}$ for the control group. The mean volume of PHE after ICH has been calculated from cranial CT. Day 1 indicates the $\mathrm{CT}$ before start of hypothermia or standard treatment. Rewarming was started at day 10 . A significant difference $(P<0.05)$ of the volumes at the specific day between the control and hypothermia group was found [5].

Volume of PHE assessed in CT: While there was no significant difference between the PHE on days 1 and 2, we found significantly higher volumes of PHE in the historic control group on days 3, 6, 11, and 14. Importantly, PHE did not increase after rewarming at days 11 and 14 .

Complications of therapeutic hypothermia: ICP crisis: none of the patients in the hypothermia group had ICP crisis, in contrast to 11 (44\%) patients in the control group.

We detected deep venous thrombosis in one patient in the hypothermia group. Shivering appeared in nine $(45 \%)$ patients and was treated sufficiently by medication including meperidine and, if needed, muscle relaxants. Pneumonia was the most common complication in hypothermia patients. Nineteen (95\%) of the patients developed pneumonia, three suffered from ARDS and sepsis. In contrast, pneumonia appeared in $79 \%$ of the control patients. Two (10\%) patients in the hypothermia group had a ventriculitis during external ventricular drainage. Five $(25 \%)$ of the hypothermia patients showed a decrease of thrombocytes. However, the thrombocyte count never dropped below $80,000 / \mu$ and no complications associated to this decrease could be detected. Four patients $(20 \%)$ developed bradycardia of below 40 beats per minute, but only one patient received treatment due to bradycardia. One patient $(5 \%)$ had a pulmonary embolism during hypothermia. However, we could not detect deep venous thrombosis or any other source for PE.

Clinical outcome: Eighteen patients survived the first 90 days after ICH. Two showed a mRS of 3, seven had a mRS of 4 , and eight a mRS of 5 after 90 days. Follow-up assessment after 1a showed that 17 patients survived $\mathrm{ICH}$. Of the surviving patients, 10 patients had a mRS of 3,12 had a mRS of 4, and two had a mRS of 5.

Discussion: PHE develops early after ICH, causes an additional mass effect after $\mathrm{ICH}$, and contributes to secondary brain injury $[3,4]$. This mass effect can be critical especially in large ICH and leads frequently to ICP crisis and brain herniation $[3,5,6]$. Therefore, PHE is an important target for therapeutic interventions. Animal experiments and clinical data show that TH is a promising candidate for edema and ICP control [4-6]. We could show in a previously published case series that TH decreased PHE and led 
Table 1(Abstract A14) Patient characteristics (from [5])

\begin{tabular}{lll}
\hline & Therapeutic hypothermia $(\boldsymbol{n}=\mathbf{2 0})$ & Historic control $(\boldsymbol{n}=\mathbf{2 5})$ \\
\hline Age (years) & $62 \pm 9$ & $67 \pm 7$ \\
GCS & $5(3$ to 10$)$ & $8(3$ to 10) \\
ICH volume on admission $(\mathrm{ml})$ & $57 \pm 25$ & $59 \pm 31$ \\
PHE on admission $(\mathrm{ml})$ & $49 \pm 40$ & $40 \pm 28$ \\
Number of patients with intraventricular hemorrhage & 13 & 14 \\
\hline
\end{tabular}

$\mathrm{ICH}$, intracerebral hemorrhage; PHE, perihemorrhagic edema.

to acceptable short-term and long-term survival $[5,6]$. Therefore, we implicated a TH as the standard care for large $\mathrm{ICH}$ in our institution and we initiated a German-Austrian controlled multicenter trial to overcome the shortcomings of our historical control group [7].

Here, we report results of the routine use of prolonged mild therapeutic hypothermia in patients suffering from large $\mathrm{ICH}$ at the level of the basal ganglia or thalamus. TH prevented the increase of PHE, prevented ICP crisis and lead to an acceptable long-term outcome compared with the historical control group. Importantly, rewarming did not lead to rebound edema as measured by CT. However, TH was also associated with complications, of which infections were the most frequent ones. As this study underscores the promising results of our proof-of-concept study, we are looking forward to the results of the $\mathrm{CINCH}$ study and other clinical data investigating the effects of temperature and temperature management of PHE in large ICH.

References

1. Broderick J, Connolly S, Feldmann E, et al: Guidelines for the management of spontaneous intracerebral hemorrhage in adults: 2007 update: a guideline from the American Heart Association/American Stroke Association Stroke Council, High Blood Pressure Research Council, and the Quality of Care and Outcomes in Research Interdisciplinary Working Group. Stroke 2007, 38:2001-2023.

2. Broderick JP, Brott TG, Duldner JE, et al: Volume of intracerebral hemorrhage: a powerful and easy-to-use predictor of 30-day mortality. Stroke 1993, 24:987-993.

3. Staykov D, Wagner I, Volbers B, Hauer EM, Doerfler AD, et al: Natural course of perihemorrhagic edema after intracerebral hemorrhage. Stroke 2011, 42:2625-2629.

4. MacLellan CL, Davies LM, Fingas MS, Colbourne F: The influence of hypothermia on outcome after intracerebral hemorrhage in rats. Stroke 2006, 37:1266-1270.

5. Kollmar R, Staykov D, Dorfler A, Schellinger PD, Schwab S, Bardutzky J: Hypothermia reduces perihemorrhagic edema after intracerebral hemorrhage. Stroke 2010, 41:1684-1689.

6. Staykov D, Schwab S, Dörfler A, Kollmar R: Hypothermia reduces perihemorrhagic edema after intracerebral hemorrhage: but does it influence functional outcome and mortality? Ther Hypotherm Temp Manage 2011, 1:105-106.

7. Kollmar R, Juettler E, Huttner HB, Dörfler A, Staykov D, Kallmuenzer B, Schmutzhard E, Schwab S, Broessner G: Cooling in intracerebral hemorrhage $(\mathrm{CINCH})$ trial: protocol of a randomized German-Austrian clinical trial. Int J Stroke 2012, 7:168-172.

\section{COOLING IN INTENSIVE CARE AND INTRAOPERATIVE MANAGEMENT}

\section{A15}

Hypothermia in burns intensive care: use of the intravenous temperature management system Thermogard XP ${ }^{\circledR}$

Meyyappan Nachiappan*, Dilnath Gurusinghe, Sameer Bhandari

Yorkshire Regional Burns Centre, Pinderfields General Hospital, Mid Yorkshire Hospitals NHS Trust, West Yorkshire, UK

Critical Care 2012, 16(Suppl 2):A15

Introduction: The care of the patient with major burns in the ICU is a complex and challenging task. They differ from the other critical care patient groups in several ways. One of the major challenges faced is confronting their hypermetabolic state and temperature management [1].
It is widely known that major burn injury is associated with the most profound of hypermetabolic responses to a pathological state. Hyperthermia that is non-infectious is a feature of the systemic inflammatory response to this. In burns intensive care, the disproportionate increase in metabolic rate to small rises in core temperature can have significant impact on resuscitation and prognosis. The pathophysiology of the hyperthermic response in major burn injury is poorly understood. It could be secondary to an infective etiology or a metabolic response to the systemic inflammation. Irrespective of the reason, sustained hyperthermia above $40^{\circ} \mathrm{C}$ can culminate in cellular injury and death [2].

The hypermetabolic response starts within the first 5 days of the major burn and can last for a year after the injury. Because of the ongoing systemic inflammatory stimulation, patients with major burns often have pyrexia and their thermoregulatory system reset at a higher baseline temperature around $38.5^{\circ} \mathrm{C}[3]$.

While therapeutic cooling is widely used in neuro intensive care in the management of hyperthermic brain-injured patients and in patients after out-of-hospital cardiac arrests, there is very scarce literature available on the management of hyperthermia in burns intensive care. We, in this article, would like to share our experience of using the intravascular temperature management system (IVTM) Thermoguard $\mathrm{XP}^{\circledR}$ in our unit to manage refractory hyperthermia in patients with major burns. We report the responses of two major burns patients to core intravascular thermoregulation during periods of severe hyperthermia $\left(>40^{\circ} \mathrm{C}\right)$.

Case 1: A 24-year-old male had sustained $80 \%$ mixed depth, total body surface area (TBSA) flame burns following a road traffic accident. He had no significant past medical history. Initial resuscitation including endotracheal intubation and fluid resuscitation was instituted in the nearby district general hospital and was transferred over to our burns ICU without much delay. On admission to the ICU, detailed assessment of the burn injuries revealed second-degree and third-degree burns involving the trunk, abdomen, back, upper and lower extremities. Initial temperature recorded was $34^{\circ} \mathrm{C}$. He responded to external warming, which included nursing in a warm environment, use of warm air blanket and warm fluids.

The patient underwent extensive escharatomies on the day of admission as a part of his initial resuscitation. He developed multiorgan failure requiring high inotropic support, renal replacement therapy and high $\mathrm{FiO}_{2}$. $\mathrm{He}$ developed hyperpyrexia (temperature $>42^{\circ} \mathrm{C}$ ) on day 11 post burn.

Relevant microbiology investigations had demonstrated no obvious focus of ongoing infection. The hyperpyrexia was resistant to conventional active cooling (bladder/gastric lavage, hemofiltration, external cooling with cooling blanket). The hyperpyrexia was associated with marked tachycardia (heart rate $>150$ beats/minute) with increasing oxygen demands and hypotension with escalating inotropic support. Forced core thermoregulation was commenced due to instability attributed to high core temperature.

The Thermogard $X P^{\oplus}$ was inserted in the femoral vein, the target temperature was set at $37^{\circ} \mathrm{C}$. Within 2 hours of initiating the IVTM, the core body temperature dropped by $3^{\circ} \mathrm{C}$ down to $39^{\circ} \mathrm{C}$. It took a further 3 hours to stabilise at the target temperature of $37^{\circ} \mathrm{C}$.

The IVTM system was used for a period of 6 days. The objective measurements of pulse rate, blood pressure, respiratory rate and urine output were seen to improve in the presence of a normothermic state (Figure 1). After a protracted and convoluted stay in the ICU, the patient was discharged to a ward after 38 days.

Case 2: A 34-year-old female, a known intravenous drug abuser, admitted to burns ICU following an attempt of deliberate self-harm. The patient, heavily drunk, allegedly grasped a high-voltage $(400 \mathrm{kV})$ live wire on a pylon and was found 15 feet away; she had sustained polytrauma 


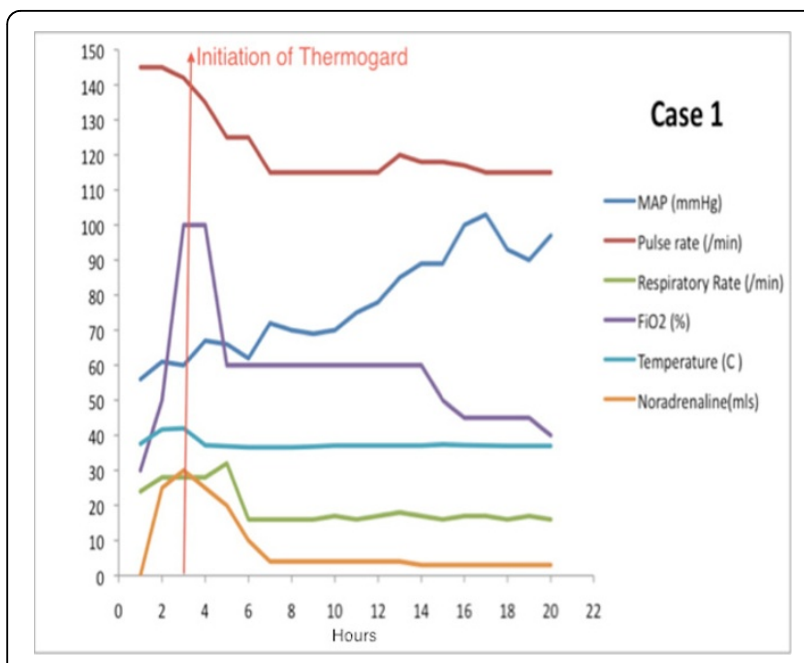

Figure 1(abstract A15) Response in vital parameters in the first 20 hours after initiating IVTM: case 1.

requiring splenectomy for splenic rupture and chest drains for pneumothoraces. She had suffered deep thermal burns involving $27 \%$ TBSA.

She had escharotomies on her both upper extremities and chest within hours of admission. Patient subsequently developed rhabdomyolysis, myoglobinuria and renal failure. The patient remained ventilated, requiring ionotropic support and haemofiltration. She remained hyperpyrexial from day 1 post burn; however, the temperature crept above $41^{\circ} \mathrm{C}$ on day 5 post burn. The temperature was persistently high despite appropriate broadspectrum antibiotics, antifungal therapy and conventional methods of cooling. Physiological instability led to the addition of forced core thermoregulation on day 8 . The target temperature of $37.5^{\circ} \mathrm{C}$ was achieved over a period of 4 to 8 hours with resultant improvement in pulse and respiratory rates and reduced inotrope levels (Figure 2 ). The forced core thermoregulation was held periodically to assess the relapse of hyperthermia and was reinstated as required.
Discussion: Thermoregulatory failure leading to elevation of core body temperature more than $37.5^{\circ} \mathrm{C}\left(37.5\right.$ to $\left.38.3^{\circ} \mathrm{C}\right)$ is hyperthermia. The core body temperature is managed in a tight range by the balance of heat production and heat loss.

The detrimental effects of hyperthermia on a patient in intensive care cannot be overstated. Hyperthermia in adults with major burns is not uncommon; however, the extent of the problem is not known. From other cohorts like patients with brain injury, it is well known that hyperthermia is an independent predictor of increased length of stay and poor outcome in the ICU [4]. The impact of hyperthermia on various organ systems and mortality depends on the degree of temperature elevation and rapidity of cooling to normal temperatures.

Among the various methods to instigate therapeutic cooling in intensive care, conventional methods could lead to treatment failure in as high as $60 \%$ of patients and the IVTM system is deemed most reliable to maintain a stable temperature [5].

Forced core thermoregulation using the IVTM system is effective in regulating labile body temperatures associated with severe burns [6]. The thermoregulation is achieved by circulation of saline via a ballooned catheter inserted into the central venous system, with automatic adjustment of saline temperature controlled by remote monitoring of patient temperature.

The downsides of using IVTM systems are the need for a central venous access and complications related to it, cost factor and also use of thermoregulatory systems may mask underlying physiological changes, potentially leading to delayed or mismanagement of any precipitants of hypothermia or hyperthermia, for example sepsis. Documentation of an artificially maintained temperature on an observation chart without reference to Thermogard $X \mathrm{P}^{\otimes}$ activity has implications for both immediate patient management and retrospective review of observation charts for audit or medico-legal purposes. Currently, facilities to download data directly from the Thermogard XP® electronic system are cumbersome and not readily available in most ward settings. We devised and used a qualitative method of documenting Thermoguard $X \mathrm{P}^{\circledR}$ activity. Firstly, $\mathrm{a}^{\prime} \mathrm{T}^{\prime}$ on the observation chart to denote the presence of a Thermogard $\mathrm{XP}^{\oplus}$ in situ. Secondly, a number assigned from a scale of +4 to -10 to represent the extent of warming or cooling, correlating with the bars displayed on the Thermogard XP ${ }^{\circledR}$ screen. These two pieces of information were documented alongside the patient's core temperature every time observations are performed. We, in addition, developed an adhesive label that replicates the cooling/warming scale displayed on the Thermogard XP ${ }^{\circledR}$ screen, allowing an arrow to be documented on the chart as depicted on the

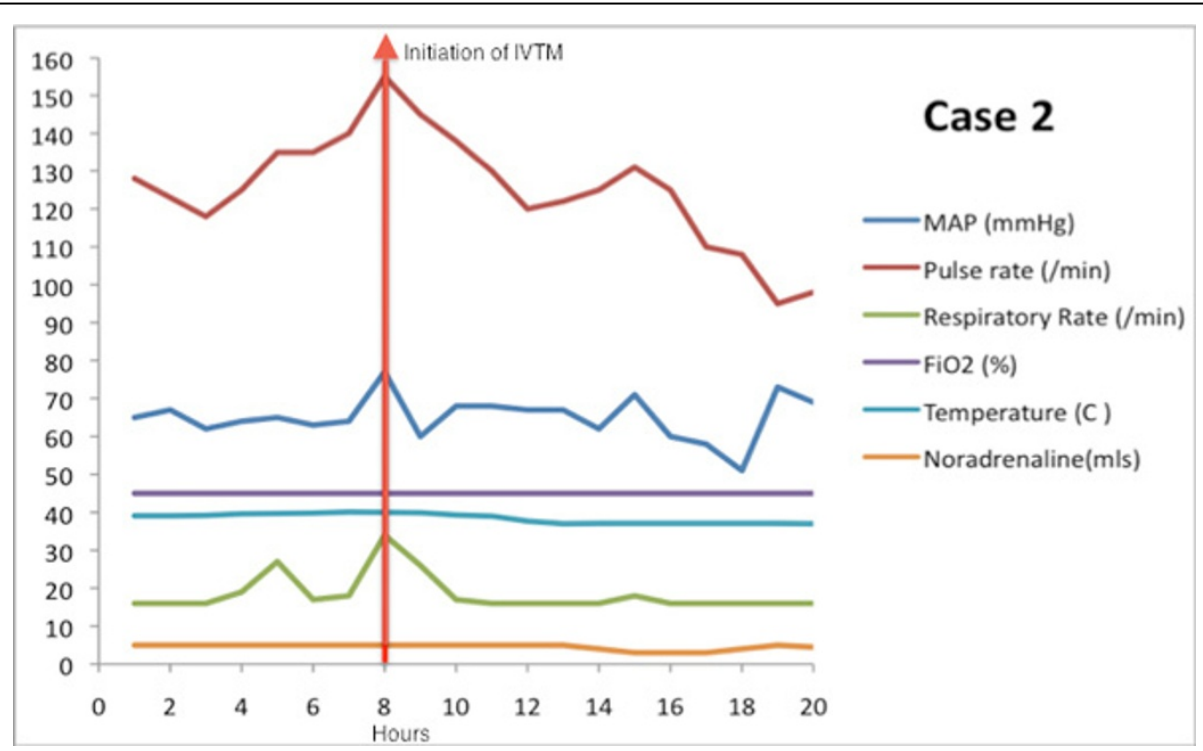

Figure 2(abstract A15) Response in vital parameters in the first 20 hours after initiating IVTM: case 2. 


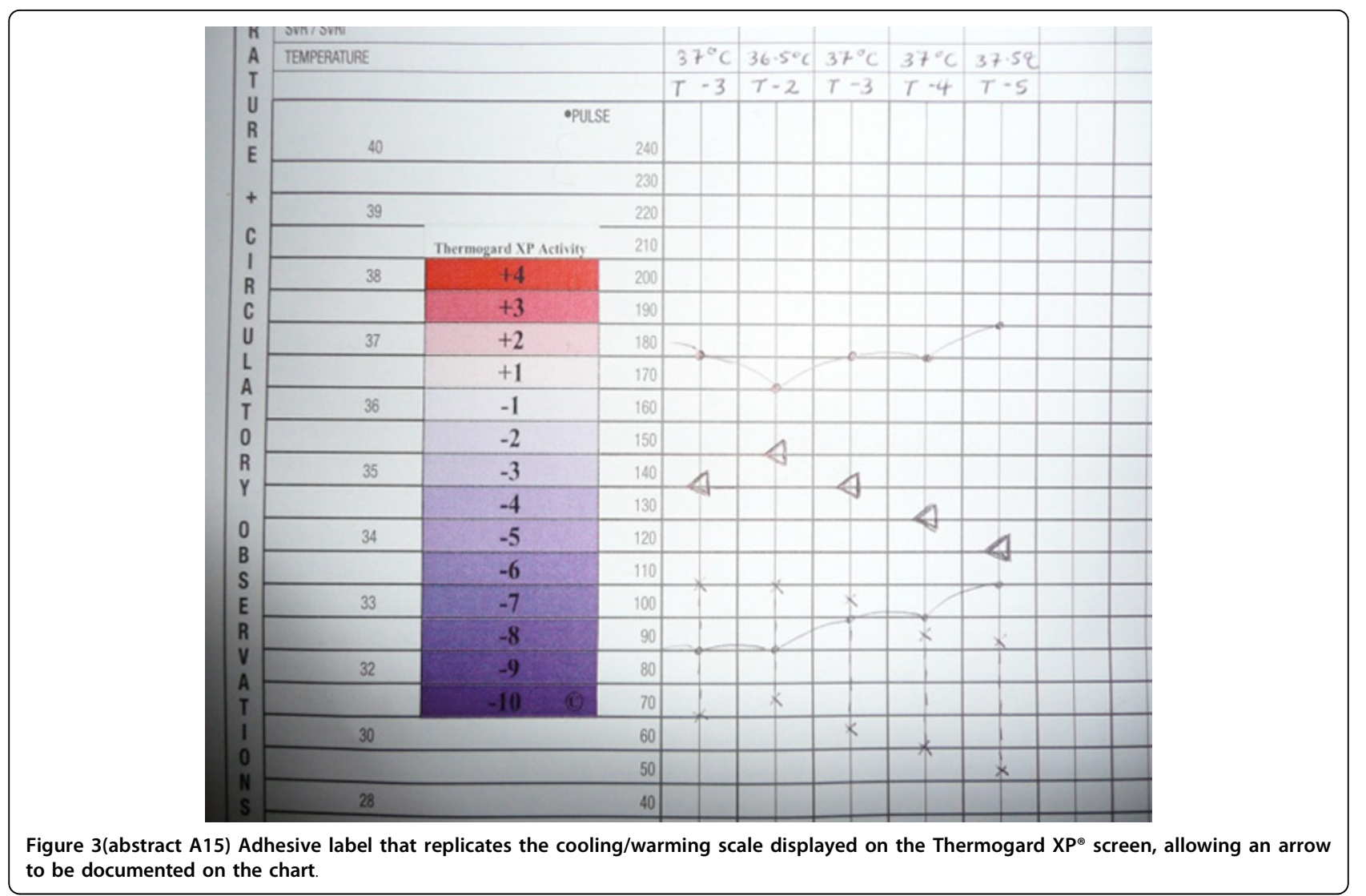

screen (Figure 3.) It provided a more visual representation of Thermogard $\mathrm{XP}^{\oplus}$ activity and was used as an adjunct to the above numerical scale. All members of the multidisciplinary team could observe our method of documenting trends in core body temperature/ Thermoguard $X P^{\oplus}$ activity at a glance. It is reproducible in any unit caring for critically ill burns patients.

Conclusion: The directed response of hyperthermia in febrile and nonfebrile states has physiological merits when considering the requirements of inflammatory mediators and cells. Forced core thermoregulation has aided us in the early management of unstable intensive care patients with refractory hyperthermia.The use of an IVTM system and subsequent documentation of artificially maintained temperature can be misleading and has implications for patient management and retrospective review.

\section{References}

1. Gunn SW, Dilbo SA: Metabolic implications of severe burn injuries and their management - a systematic review of the literature. World I Surg 2008, 32:1857.

2. Lepock JR: Cellular effects of hyperthermia: relevance to the minimum dose for thermal damage. Int I Hyperthermia 2003, 19:252-266.

3. Greenhalgh DG, Saffle JR, Holmes JH, et al: American Burn Association consensus con ference to define sepsis and infection in burns. J Burn Care Res 2007, 28:776-790.

4. Diringer MN, Reaven NL, Funk SE, Uman GC: Elevated body temperature independently contributes to increased length of stay in neurologic intensive care unit patients. Crit Care Med 2004, 32:1489-1495.

5. Hoedemaekers CW, Ezzahti M, Gerritsen A, van der Hoeven JG: Comparison of cooling methods to induce and maintain normo- and hypothermia in intensive care unit patients: a prospective intervention study. Crit Care 2007, 11:R91.

6. Mavrogordato AE, Wagstaff MJD, Fletcher AJP, Wilson DI, Jayamaha JEL: A novel method to treat hyperthermia in a burns case: use of a catheterbased heat exchange system. Burns 2009, 35:141-145.

\section{A16}

Hypothermia after aneurysmal subarachnoid hemorrhage

Martin Seule*, Emanuela Keller

Neurointensive Care Unit, Department of Neurosurgery, University Hospital Zurich, Switzerland

Critical Care 2012, 16(Suppl 2):A16

Introduction: Aneurysmal subarachnoid hemorrhage (SAH) is a common and frequently devastating condition, accounting for 1 to $7 \%$ of all strokes with an incidence of 9.1 per 100,000 [1]. Major advances in SAH management over the past three decades have decreased case fatality by $0.8 \%$ per year, but it is still $40 \%$ and many survivors have long-term disabilities [2]. The most important and potentially treatable complication is development of delayed cerebral ischemia (DCI), which can progress to cerebral infarction associated with poor outcome.

The pathogenesis of $\mathrm{DCl}$ is multifactorial and assumed to be initiated in the early phase of SAH [3]. The onset of SAH is characterized by a shortlasting and cerebral perfusion pressure (CPP)-dependent decrease in cerebral blood flow (CBF) leading to global cerebral ischemia [4]. Elevated intracranial pressure (ICP) and acute cerebral ischemia are the main factors for early disruption of the blood-brain barrier as well as impairment of autoregulation associated with brain edema and brain swelling. Important pathogenic mechanisms of CPP-independent hypoperfusion include acute vasoconstriction, cortical spreading ischemia, and activation of the inflammatory response. The release of oxyhemoglobin and endothelin-1 (ET-1) are the key factors for cortical spreading ischemia, reduced nitric oxide (NO) availability, and secondary cytotoxic edema formation. Cerebral vasospasm (CVS) is a delayed morphological narrowing of cerebral arteries, occurring 4 to 10 days after SAH. Although CVS have been associated with $\mathrm{DCl}$, it is generally accepted that CVS is not solely responsible for DCI [5]. In fact, DCI may occur in the absence of CVS and vice versa and the distribution of 
CVS may fail to reliably predict the subsequent pattern of cerebral infarction [6].

Neuroprotective strategies to prevent $\mathrm{DCl}$ have been mainly focused on treatment of CVS, but despite extensive research, effective and/or causative prophylaxis and treatment are not available [7]. So far, oral nimodipine is the only drug that can reduce the incidence of $\mathrm{DCl}$ and poor outcome, but there is no beneficial effect on CVS [8]. Hypothermia (HT) treatment exerting numerous protective effects such as a decrease in cerebral metabolism [9], stabilization of the blood-brain barrier [10], reduction of cerebral edema [11], suppression of excitatory neurotransmitter concentrations [12] and inflammatory reactions [13] seems to be well suited as a neuroprotective strategy. In the following, the clinical application of HT after SAH is presented by reviewing the existing literature.

Hypothermia during aneurysm surgery: In the past, promising studies on intraoperative HT during aneurysm surgery as an attempt to reduce ischemic injury have been published [14-18]. The Intraoperative Hypothermia for Aneurysm Surgery Trial (IHAST) applied HT in a randomized study in 1,001 patients with good-grade SAH (WFNS 1 to 3); however, it found no improvement in neurological outcome 3 months after surgery [19]. Post-hoc analysis demonstrated no difference in the incidence of cognitive impairment between hypothermic and normothermic groups [20]. Furthermore, there was no evidence for the benefit of intraoperative HT on 24-hour and 3-month outcome in patients who underwent temporary clipping [21]. It has to be noted that these results only apply to good-grade SAH patients and may not be extrapolated to the general SAH population. This suggests that only a carefully selected subgroup of patients, with specific complications induced by $\mathrm{SAH}$, may benefit from $\mathrm{HT}$ treatment at a particular time and for certain duration.

Hypothermia in patients with poor-grade subarachnoid hemorrhage: Several experimental studies have demonstrated that $\mathrm{HT}$ is effective in minimizing neuronal damage, if induced before or early after the SAH. Kawamura and colleagues found a reduced expression of c-jun and hsp-70 mRNA, indicating a reduced stress response that may otherwise manifest as necrosis or apoptosis [22]. Thome and colleagues and Schubert and colleagues demonstrated that early induction of $\mathrm{HT}$, up to 60 minutes after $\mathrm{SAH}$, reversed CPP-independent hypoperfusion and brain edema formation, preserved cerebral autoregulation, and reduced accumulation of lactate and glutamate $[11,12,23]$. On the other hand, delayed induction of $\mathrm{HT}$, up to 180 minutes after SAH, failed to reduce brain edema formation, thus indicating a limited time window of HT application [24]. However, HT was effective in reducing ICP and associated with improved neurological recovery. These studies encourage early induction of $\mathrm{HT}$ in poor-grade SAH patients, for example, as soon as signs of brain swelling are seen on CT scans.

So far, only few retrospective case series have investigated the effect of mild HT during the acute phase of SAH. Table 1 presents an overview of these studies applying $\mathrm{HT}$ within 24 hours after $\mathrm{SAH}$ to patients with poor-grade SAH (WFNS 4 to 5). The duration of HT varied between 2 and 14 days. Overall outcome results were unsatisfactory with a mortality rate of $47.4 \%$ and favorable outcome in less than one-quarter of cases. Yasui and colleagues evaluated seven patients admitted within 6 hours from symptom onset and treated with HT for 48 hours [25]. PET studies during HT revealed a reduction in cerebral oxygen metabolism exceeding the decrease in $C B F$, thus indicating a state of luxury perfusion. However, according to the
Barthel Index only two patients were independent, one was partially dependent, able to walk without assistance, and more than one-half of the patients bedridden at 12 months after SAH. Anei and colleagues compared outcome results before and after introduction of HT treatment to their institution and found no significant difference in the mortality rate with $56.3 \%$ and $57.9 \%$, respectively [26]. The authors noted that post-HT fever can be a serious complication resulting in brain swelling and unfavorable outcome. In contrast to these studies, Gasser and colleagues showed more promising outcome results in 21 patients with poor-grade SAH (WFNS 4 to 5) and induction of $\mathrm{HT}$ after developing intracranial hypertension (>15 $\mathrm{mmHg}$ ) that was refractory to conventional treatment. HT was induced on average $4.2 \pm 3.3$ days after SAH and maintained for $4.3 \pm 3.9$ days. Prolonged HT ( $>72$ hours) was associated with an increased risk of systemic complications, but 10 patients (47.6\%) showed favorable outcome (GOS 4 to 5) and five patients died (23.8\%).

Hypothermia in patients with delayed cerebral ischemia/cerebral vasospasm: Recently, a SAH-CVS model in dogs demonstrated that HT can attenuate the degree of CVS up to 14 days after SAH, possibly by regulating the levels of ET-1 and NO [27]. The duration of HT was directly proportional to the duration of relieving CVS. Table 2 presents an overview of clinical studies applying HT to patients with symptomatic CVS leading to $\mathrm{DCl}$. Nagao and colleagues treated five patients with good-grade SAH (H\&H I to III), starting HT either during delayed aneurysm clipping or if CVS was refractory to hyperdynamic and endovascular therapy [28]. Four patients survived with favorable outcome and one patient was severely disabled. In a follow-up study, Nagao and colleagues included eight patients with good-grade SAH (H\&H II to III), and seven patients had favorable outcome and one survived severely disabled [29]. According to SPECT studies, HT was associated with decreased CBF levels in all patients. Nakamura and colleagues reported a reduction in arterio-jugular oxygen difference $\left(\mathrm{AVDO}_{2}\right)$ during $\mathrm{HT}$ in five patients (H\&H III to IV), thus indicating a reduced metabolic demand [30]. All patients received hyperdynamic therapy before and during $\mathrm{HT}$, but outcome results were unsatisfactory. Possible contributing factors of poor outcome include a higher grade of SAH and it is unclear whether endovascular treatment was applied or not. In our series of $100 \mathrm{SAH}$ patients treated with $\mathrm{HT}, 28$ patients had symptomatic CVS refractory to hypertensive therapy and endovascular treatment [31]. HT was combined with barbiturate coma in 23 of 28 patients and maintained until CVS resolved or severe side effects occurred (mean duration $5.7 \pm 3.3$ days). Although the majority of patients had poor-grade SAH (H\&H 4 to 5 in $57.1 \%$, Fisher 3 to 4 in $85.7 \%$ ), favorable outcome (GOS 4 to 5 ) was achieved in $57.1 \%$. In patients with intracranial hypertension $(>20 \mathrm{mmHg})$ with and without refractory CVS, favorable outcome was obtained in only $25.0 \%$ and $26.5 \%$, respectively. Systemic side effects possibly caused from HT and/or barbiturate coma included pneumonia in $52.0 \%$, thrombocytopenia $(<100,000 / \mu \mathrm{l})$ in $47.0 \%$, septic shock syndrome in $40.0 \%$, and acute respiratory distress syndrome in $16.0 \%$. In a subgroup of seven patients with combined $\mathrm{HT}$ and barbiturate coma, daily levels of IL-6, IL-1 $\beta, T N F \alpha$, and leukocyte count in the cerebrospinal fluid and plasma were quantified [13]. IL-6 levels in the cerebrospinal fluid and systemic IL-1 $\beta$ levels were significantly lower compared with patients receiving barbiturate coma alone $(n=8)$, thus indicating HT-related attenuation of the inflammatory response.

\section{Table 1(Abstract A16) Studies applying hypothermia on day of aneurysm rupture to patients with poor-grade SAH} (WFNS 4 to 5 or H\&H IV to V)

\begin{tabular}{llllll}
\hline Study & $\begin{array}{l}\text { Number of } \\
\text { patients }(\boldsymbol{n})\end{array}$ & $\begin{array}{l}\text { Target temperature } \\
\left({ }^{\circ} \mathbf{C}\right)\end{array}$ & $\begin{array}{l}\text { Duration of hypothermia } \\
\text { (days) }\end{array}$ & $\begin{array}{l}\text { Favorable outcome*, } \\
\%(\boldsymbol{n})\end{array}$ & $\begin{array}{l}\text { Mortality rate, \% } \\
(\boldsymbol{n})\end{array}$ \\
\hline $\begin{array}{l}\text { Nagao and colleagues } \\
(2000)\end{array}$ & 9 & 32 to 34 & 5 to 14 & $44.4 \%(4)$ & $55.6 \%(5)$ \\
$\begin{array}{l}\text { Yasui and colleagues } \\
(2002)\end{array}$ & 7 & 33 to 34 & 2 & $42.8 \%(3)^{*}$ & $0.0 \%(0)$ \\
$\begin{array}{l}\text { Nakamura and colleagues } \\
(2002)\end{array}$ & 3 & 32 to 34 & 5 to 14 & $0.0 \%(0)$ & $66.7 \%(2)$ \\
Anei and colleagues (2010) & 19 & 34 & 2 & $5.3 \%(1)$ & $57.9 \%(11)$ \\
Total & 38 & & & $21.1 \%(8)$ & $47.4 \%(18)$ \\
\hline
\end{tabular}

${ }^{*} \mathrm{GOS} \geq 4$ or $\mathrm{mRS} \leq 3$. 


\section{Table 2(Abstract A16) Studies applying hypothermia to patients with symptomatic vasospasm refractory to} conventional treatment

\begin{tabular}{llllll}
\hline Study & $\begin{array}{l}\text { Number of } \\
\text { patients }(\boldsymbol{n})\end{array}$ & $\begin{array}{l}\text { Target temperature } \\
\left({ }^{\circ} \mathbf{C}\right)\end{array}$ & $\begin{array}{l}\text { Duration of hypothermia } \\
(\text { days })\end{array}$ & $\begin{array}{l}\text { Favorable outcome* } \\
\%(\boldsymbol{n})\end{array}$ & $\begin{array}{l}\text { Mortality rate, \% } \\
(\boldsymbol{n})\end{array}$ \\
\hline $\begin{array}{l}\text { Nagao and colleagues } \\
(2000)\end{array}$ & 5 & 32 to 34 & 5 to 14 & $80.0 \%(4)$ & $0.0 \%(0)$ \\
$\begin{array}{l}\text { Nakamura and colleagues } \\
(2002)\end{array}$ & 5 & 32 to 34 & 5 to 14 & $40.0 \%(2)$ & $60.0 \%(3)$ \\
$\begin{array}{l}\text { Nagao and colleagues } \\
(2003)\end{array}$ & 8 & 32 to 34 & 5 to 12 & $87.5 \%(7)$ & $0.0 \%(0)$ \\
$\begin{array}{l}\text { Seule and colleagues } \\
(2009)\end{array}$ & 28 & 33 to 34 & 1 to 16 & $57.1 \%(16)$ & $28.6 \%(8)$ \\
Total & 46 & & & $63.0 \%(29)$ & $26.1 \%(12)$ \\
\hline
\end{tabular}

Note: ${ }^{*} \mathrm{GOS} \geq 4$ or $\mathrm{mRS} \leq 3$.

Conclusion: So far, the evidence of HT on improved outcome after SAH is limited. Intraoperative HT has been abandoned based on the randomized Intraoperative Hypothermia Study on Aneurysm Surgery in good-grade SAH patients. The available data suggest that HT may improve outcome in a carefully selected subgroup of patients developing intracranial hypertension and/or symptomatic CVS that are refractory to conventional treatment. Further evaluation of cerebral hemodynamics and oxygenation during $\mathrm{HT}$ treatment is required to obtain important insights in the effects of HT and to identify patients who may benefit most.

References

1. Feigin $V L$, Lawes $C M$, Bennett DA, Anderson CS: Stroke epidemiology: a review of population-based studies of incidence, prevalence, and casefatality in the late 20th century. Lancet Neurol 2003, 2:43-53.

2. Nieuwkamp DJ, Setz LE, Algra A, Linn FH, de Rooij NK, Rinkel GJ: Changes in case fatality of aneurysmal subarachnoid haemorrhage over time, according to age, sex, and region: a meta-analysis. Lancet Neurol 2009, 8:635-642.

3. Ostrowski RP, Colohan AR, Zhang JH: Molecular mechanisms of early brain injury after subarachnoid hemorrhage. Neurol Res 2006, 28:399-414.

4. Bederson JB, Germano IM, Guarino L: Cortical blood flow and cerebral perfusion pressure in a new noncraniotomy model of subarachnoid hemorrhage in the rat. Stroke 1995, 26:1086-1091, discussion 1091-1092.

5. Vergouwen MD, llodigwe D, Macdonald RL: Cerebral infarction after subarachnoid hemorrhage contributes to poor outcome by vasospasmdependent and -independent effects. Stroke 2011, 42:924-929.

6. Rabinstein AA, Weigand S, Atkinson JL, Wijdicks EF: Patterns of cerebral infarction in aneurysmal subarachnoid hemorrhage. Stroke 2005, 36:992-997.

7. Muroi C, Seule M, Mishima K, Keller E: Novel treatments for vasospasm after subarachnoid hemorrhage. Curr Opin Crit Care 2012, 18:119-126.

8. Castanares-Zapatero D, Hantson P: Pharmacological treatment of delayed cerebral ischemia and vasospasm in subarachnoid hemorrhage. Ann Intensive Care 2011, 1:12.

9. Erecinska M, Thoresen M, Silver IA: Effects of hypothermia on energy metabolism in mammalian central nervous system. J Cereb Blood Flow Metab 2003, 23:513-530.

10. Karibe H, Zarow GJ, Graham SH, Weinstein PR: Mild intraischemic hypothermia reduces postischemic hyperperfusion, delayed postischemic hypoperfusion, blood-brain barrier disruption, brain edema, and neuronal damage volume after temporary focal cerebral ischemia in rats. J Cereb Blood Flow Metab 1994, 14:620-627.

11. Schubert GA, Poli S, Schilling L, Heiland S, Thome C: Hypothermia reduces cytotoxic edema and metabolic alterations during the acute phase of massive SAH: a diffusion-weighted imaging and spectroscopy study in rats. J Neurotrauma 2008, 25:841-852.

12. Schubert GA, Poli S, Mendelowitsch A, Schilling L, Thome C: Hypothermia reduces early hypoperfusion and metabolic alterations during the acute phase of massive subarachnoid hemorrhage: a laser-Doppler-flowmetry and microdialysis study in rats. J Neurotrauma 2008, 25:539-548.

13. Muroi C, Frei K, El Beltagy M, Cesnulis E, Yonekawa Y, Keller E: Combined therapeutic hypothermia and barbiturate coma reduces interleukin- 6 in the cerebrospinal fluid after aneurysmal subarachnoid hemorrhage. J Neurosurg Anesthesiol 2008, 20:193-198.

14. Hindman BJ, Todd MM, Gelb AW, Loftus CM, Craen RA, Schubert A, Mahla ME, Torner JC: Mild hypothermia as a protective therapy during intracranial aneurysm surgery: a randomized prospective pilot trial. Neurosurgery 1999, 44:23-32, discussion 32-33.

15. Karibe H, Sato K, Shimizu H, Tominaga T, Koshu K, Yoshimoto T: Intraoperative mild hypothermia ameliorates postoperative cerebral blood flow impairment in patients with aneurysmal subarachnoid hemorrhage. Neurosurgery 2000, 47:594-599, discussion 599-601.

16. Ogilvy CS, Carter BS, Kaplan S, Rich C, Crowell RM: Temporary vessel occlusion for aneurysm surgery: risk factors for stroke in patients protected by induced hypothermia and hypertension and intravenous mannitol administration. J Neurosurg 1996, 84:785-791.

17. Sato K, Yoshimoto T: Systemic and cerebral haemodynamics during craniotomy under mild hypothermia in patients with acute subarachnoid haemorrhage. Acta Neurochir (Wien) 2000, 142:1013-1019, discussion 1019-1020.

18. Kimme P, Fridrikssen S, Engdahl O, Hillman J, Vegfors M, Sjoberg F: Moderate hypothermia for 359 operations to clip cerebral aneurysms. $\mathrm{Br}$ J Anaesth 2004, 93:343-347.

19. Todd MM, Hindman BJ, Clarke WR, Torner JC: Mild intraoperative hypothermia during surgery for intracranial aneurysm. N Engl J Med 2005, 352:135-145.

20. Anderson SW, Todd MM, Hindman BJ, Clarke WR, Torner JC, Tranel D, Yoo B, Weeks J, Manzel KW, Samra S: Effects of intraoperative hypothermia on neuropsychological outcomes after intracranial aneurysm surgery. Ann Neurol 2006, 60:518-527.

21. Hindman BJ, Bayman EO, Pfisterer WK, Torner JC, Todd MM: No association between intraoperative hypothermia or supplemental protective drug and neurologic outcomes in patients undergoing temporary clipping during cerebral aneurysm surgery: findings from the Intraoperative Hypothermia for Aneurysm Surgery Trial. Anesthesiology 2010, 112:86-101.

22. Kawamura Y, Yamada K, Masago A, Katano H, Matsumoto T, Mase M: Hypothermia modulates induction of hsp70 and c-jun mRNA in the rat brain after subarachnoid hemorrhage. J Neurotrauma 2000, 17:243-250.

23. Thome C, Schubert G, Piepgras A, Elste V, Schilling L, Schmiedek P: Hypothermia reduces acute vasospasm following SAH in rats. Acta Neurochir Suppl 2001, 77:255-258

24. Torok E, Klopotowski M, Trabold R, Thal SC, Plesnila N, Scholler K: Mild hypothermia $\left(33^{\circ} \mathrm{C}\right)$ reduces intracranial hypertension and improves functional outcome after subarachnoid hemorrhage in rats. Neurosurgery 2009, 65:352-359, discussion 359.

25. Yasui N, Kawamura S, Suzuki A, Hadeishi H, Hatazawa J: Role of hypothermia in the management of severe cases of subarachnoid hemorrhage. Acta Neurochir Suppl 2002, 82:93-98.

26. Anei $R$, Sakai $H$, lihara K, Nagata I: Effectiveness of brain hypothermia treatment in patients with severe subarachnoid hemorrhage: comparisons at a single facility. Neurol Med Chir (Tokyo) 2010, 50:879-883.

27. Wang ZP, Chen HS, Wang FX: Influence of plasma and cerebrospinal fluid levels of endothelin-1 and NO in reducing cerebral vasospasm after subarachnoid hemorrhage during treatment with mild hypothermia, in a dog model. Cell Biochem Biophys 2011, 61:137-143. 
28. Nagao S, Irie K, Kawai N, Kunishio K, Ogawa T, Nakamura T, Okauchi M: Protective effect of mild hypothermia on symptomatic vasospasm: a preliminary report. Acta Neurochir Supp/ 2000, 76:547-550.

29. Nagao S, Irie K, Kawai N, Nakamura T, Kunishio K, Matsumoto Y: The use of mild hypothermia for patients with severe vasospasm: a preliminary report. J Clin Neurosci 2003, 10:208-212.

30. Nakamura T, Tatara N, Morisaki K, Kawakita K, Nagao S: Cerebral oxygen metabolism monitoring under hypothermia for severe subarachnoid hemorrhage: report of eight cases. Acta Neurol Scand 2002, 106:314-318.

31. Seule MA, Muroi C, Mink S, Yonekawa Y, Keller E: Therapeutic hypothermia in patients with aneurysmal subarachnoid hemorrhage, refractory intracranial hypertension, or cerebral vasospasm. Neurosurgery 2009, 64:86-92, discussion 92-93.

A17

Hypothermia in the operating theatre

Andreas Gruber ${ }^{1 *}$, Wilhelm Behringer ${ }^{2}$, Engelbert Knosp

${ }^{1}$ Department of Neurosurgery, Medical University of Vienna, Austria;

${ }^{2}$ Department of Emergency Medicine, Medical University of Vienna, Austria

Critical Care 2012, 16(Suppl 2):A17

Background: When addressing neuroprotective effects and possible indications of therapeutic hypothermia, several scenarios have to be distinguished. First, therapeutic hypothermia can be classified based on the depth of cooling from normal body temperature into mild hypothermia $\left(32\right.$ to $\left.35^{\circ} \mathrm{C}\right)$, moderate hypothermia $\left(28\right.$ to $32^{\circ} \mathrm{C}$ ), deep hypothermia ( 28 to $\left.17^{\circ} \mathrm{C}\right)$, and profound $\left(<17^{\circ} \mathrm{C}\right)$. Second, therapeutic hypothermia can be initiated prior to the insult, for preservation of tissue during the insult, or for the reduction of reperfusion injury after an insult. Third, animal studies and clinical trials have addressed the neuroprotective effects of therapeutic hypothermia under conditions of both global and focal ischaemia. Forth, additional parameters, for example, rewarming rate, duration of ischaemia, and extent of reperfusion, influence the effects of therapeutic hypothermia. Fifth, different outcome measures have been used to describe the effects of hypothermia, for example, infarct size, extent of cellular death, and neurologic condition at different time intervals after ischemia. Therefore, whenever discussing procedures involving therapeutic hypothermia, the aforementioned parameters deserve clarification.

Cerebral ischaemia: Cerebral ischaemia results from a reduction or complete loss of cerebral blood flow (CBF) and lack of cerebral oxygenation, followed by depletion of ATP, dysfunction of ATP-dependent membrane pumps and subsequently occurrence of anoxic depolarisation. A large amount of glutamate is released from the intracellular space into the extracellular space, causing excitotoxic injury by stimulating $N$-methyl-Daspartate (NMDA) receptors and triggering calcium influx. Increased intracellular calcium levels per se amplify injury by increasing calcium permeability and glutamate release via second messenger mechanisms. These acute cascades lead to necrotic neuronal death by interfering with the mitochondrial respiratory chain. Ischaemia and reperfusion further enhance excitotoxicity by providing oxygen as a substrate for several enzymatic oxidation reactions, thereby generating products of reactive oxygen species in large quantities. These free radicals enhance protein oxidation and lipid membrane disintegration and in conjunction with bloodbrain barrier (BBB) disruption further contribute to ischaemic necrosis. Apoptosis also occurs in cerebral ischemia, with antiapoptotic proteins being selectively upregulated in surviving neurons and proapoptotic proteins being highly expressed in dying cells.

Hypothermia in cerebral ischaemia: The first controlled attempts to cool the human brain were undertaken by the neurosurgeon Temple Fay in 1938 [1]. Irrigating the brain directly with ice water and sometimes achieving solid parenchymal freezing, he claimed 'extremely gratifying results' in a paper on 'local and generalized refrigeration of the human brain'. Over time, many mechanisms have been proposed regarding the neuroprotective effect of hypothermia. First, hypothermia results in a temperature-dependent decrease of oxygen and glucose metabolism; that is, a $10^{\circ} \mathrm{C}$ decrease in temperature reduces ATP consumption and the cerebral metabolic rate (CMR) of oxygen, glucose, and lactate twofold to fourfold [2]. Second, intraischaemic hypothermia exerts inhibitory effects on many of the detrimental ischaemic cascades, thereby retarding the initial ATP depletion, preserving metabolic stores, delaying anoxic depolarisation, reducing ischaemiainduced excitotoxic neurotransmitter release and intracellular calcium levels, changing glutamate receptor regulation, and limiting BBB breakdown. Busto and coworkers in 1987 reported that even 1 to $2^{\circ} \mathrm{C}$ temperature reductions were sufficient to protect against experimental ischemic stroke [3], thereby demonstrating that the aforementioned mechanisms can exceed the effects of temperature-induced reductions in CMR, and in turn providing the pathophysiologic foundation for mild and moderate therapeutic hypothermia in the management of cerebral ischaemia.

Indications for therapeutic hypothermia: The importance of therapeutic hypothermia has recently been emphasized by randomised trials in patients with global cerebral ischaemia from out-of-hospital cardiac arrest (OHCA) [4] and in neonates with perinatal hypoxic-ischaemic encephalopathy (HIE) [5] Clinical experience with patients suffering traumatic brain injuries (TBI) and ischaemic strokes are not as convincing, although mild therapeutic hypothermia was sufficient to control intracranial hypertension in this population.

The use of therapeutic hypothermia in today's OR theatres differs significantly from the scenarios outlined in the context of OHCA, HIE, TBI, or stroke. First, in the OR theatre hypothermia is induced beforehand in expectation of a severe cerebral ischaemic challenge caused by the surgical procedure. Second, underexperienced neuro-anaesthesiologist and cardio-anaesthesiologic management, highly invasive procedures including deep hypothermic cardiac arrest (DHCA) and selective cerebral perfusion can be employed. In other words, whereas outside the OR mild to moderate hypothermia is used to treat patients in the post-ischaemic period, deep intra-ischaemic hypothermia can be used in cardiothoracic and neurosurgery for the management of congenital heart disease, thoracic aneurysms, and intracranial aneurysms; that is, interventions provoking global cerebral ischaemia and otherwise resulting in devastating intraoperative strokes. Moreover, mild hypothermia is used during temporary parent artery clipping in cerebral aneurysm surgery, closely resembling a state of mild intra-ischaemic hypothermia in a condition of transient focal cerebral ischaemia.

DHCA in global cerebral ischaemia: Although DHCA is being used in both cardiothoracic and neurosurgery and usually involves the same principles of extracorporeal circulation under cardiopulmonary bypass $(\mathrm{CPB})$, the rationale behind these procedures is very different.

Hypothermic CPB and DHCA are established strategies of cerebroprotection during cardiothoracic surgery. Cerebral circulatory standstill is an undesired byproduct of this procedure and limits the possible safe duration of surgery. To overcome this problem, methods of retrograde and antegrade cerebral perfusion have been established [6]. Retrograde cerebral perfusion (RCP) was initially considered to extend the safe operative time by both, backward perfusion of the brain via the superior cava vein at pressures of 20 to $30 \mathrm{mmHg}$, and selective cooling of the brain parenchyma. It has become evident that RCP indeed provides inadequate cerebral perfusion and exerts neuroprotective actions mainly by providing additional cerebral cooling. In contrast, antegrade cerebral perfusion (ACP) is obtained by intermittent infusion of cooled blood directly into cerebral arteries. In detail, selective ACP allows bihemispheric perfusion through direct cannulation of at least two aortic arch vessels, whereas nonselective, hemispheric ACP uses the axillary canula that is used for systemic perfusion as a route for ACP. From the neurosurgical standpoint, not surprisingly, insufficient crossflow across the communicating arteries at the circle of Willis with inadequate perfusion of the contralateral hemisphere has been identified as an important limitation for nonselective ACP. Although a final cooling temperature of $20^{\circ} \mathrm{C}$ or below with a long cooling time and gradual rewarming are commonly advocated, promising results have been reported by some groups using selective ACP with moderate hypothermia [7].

From the neurosurgical standpoint, the most important information from the cardiothoracic surgical experience is the recommendation that the DHCA time in sole application should not exceed 20 to 25 minutes and in every case with expected DHCA time $>25$ minutes, ACP or RCP supplement should be performed. In the field of neurosurgery, DHCA combines advanced cerebroprotection with optimal surgical conditions; that is, a blood-free noflow surgical field and a collapsed aneurysm dome. Since the surgical procedure requires cerebral exsanguination for rapid aneurysm repair, ACP and RCP cannot be employed to dilate the time of DHCA, limiting the safe no-flow period to 20 to 30 minutes.

Management algorithms for intracranial aneurysm surgery under DHCA have been published previously [8-11]. Such advanced cerebrovascular procedures require multimodality neuromonitoring and are performed under barbiturate-induced EEG burst suppression. The decision to use 
DHCA is usually made intraopertively; that is, hypothermia and cardiac standstill are employed only after microsurgical exploration has proven that safe aneurysm repair is impossible without these adjunctive measures. In this case, brain retractor placement is adjusted, avoiding further retractor repositioning - with the risk of contusional parenchymal haemorrhage - after systemic heparinisation. Thereafter femoro-femoral percutaneous cannulation is performed and after systemic heparinisation (300 to $400 \mathrm{IU} / \mathrm{kg}$ ) heart-lung extracorporeal circulation with a heat exchanger and oxygenator is started. Once adequate CPB flow is achieved, systemic hypothermia is induced. Cooling during extracorporeal circulation is continued until a desired brain temperature of 14 to $18^{\circ} \mathrm{C}$ is reached. Hypothermia results in ventricular fibrillation below $28^{\circ} \mathrm{C}$ and circulatory arrest at 18 to $22^{\circ} \mathrm{C}$. At this point CPB is stopped and blood is actively drained into the venous reservoir, rendering the operative field blood free and the aneurysm collapsed. The duration of this circulatory standstill is limited to the duration of surgical aneurysm repair. In the largest reported series [9], the mean duration of circulatory arrest was 21.8 minutes (range 2 to 72 minutes) with a mean temperature during circulatory arrest of $17.2^{\circ} \mathrm{C}$ (range 12 to $20^{\circ} \mathrm{C}$ ). The decompressed aneurysm can now be dissected circumferentially, adjacent vital perforating branches can be separated from the aneurysm fundus, and in partially thrombosed lesions the sac can be opened and evacuated. Some centres use short but repetitive periods of circulatory standstill followed by periods of reperfusion, thereby reducing the length of a single ischaemic period and checking for bleedings in the operative field [12]. After aneurysm repair, circulation is restored and under slow rewarming the heart starts to fibrillate spontaneously and will either convert to sinus rhythm or will require cardioversion. Pharmacologic peripheral vasodilation is used to facilitate reperfusion, the blood previously drained is reinfused, and heparin is reversed with protamine. Only after reperfusion, rewarming, and reversal of heparin is adequate hemostasis possible in the operative field.

DHCA was used to treat intracranial aneurysms in the 1960s, but shortcomings of CPB and hypothermia-induced coagulopathies resulted in operative morbidity and declining use of the procedure. In the late 1980s, refined microsurgical and anaesthesiologic techniques allowed the use of DHCA for the management of most difficult intracranial aneurysms in selected neurosurgical centres of excellence [8-11]. With the development of new diagnostic and therapeutic strategies, however, DHCA is only rarely needed for the management of cerebral aneurysms today. Possible explanations include the following. First, easy access to improved neuroradiologic imaging techniques has resulted in the detection of cerebral aneurysms at an earlier stage; that is, before they can grow to giant size requiring the aforementioned surgical procedures. Second, endovascular treatment, for example, flow diverter implantation [13] and techniques of flow reversal, has become an alternative to surgery and should in some instances be considered the first-line treatment; for example, complex and giant posterior circulation aneurysms as well as patients with significant co-morbidities. Third, techniques of cerebral revascularisation that is, parent artery occlusion under bypass protection - have proven highly effective in the management of complex aneurysms [14]. Forth, alternative strategies for intraoperative aneurysm decompression have been discovered. Adenosine-induced cardiac asystole has emerged as a possible alternative, resulting in brief repeated periods of cardiac arrest ( 5 to 15 seconds) to facilitate aneurysm clipping [15]. Others have used selective brain cooling with extracorporeal femoral to carotid artery perfusion in giant aneurysm surgery [16], a technique differing from DHCA in so far as the patient's body core temperature is maintained at $35^{\circ} \mathrm{C}$ and aneurysm decompression is achieved by temporary clipping (trapping) of the parent artery under selective brain cooling to $22^{\circ} \mathrm{C}$. Another group has reported successful treatment of a giant basilar artery aneurysm using moderate hypothermia and extracorporeal circulation [12].

Even in expert hands, the procedural complication rate of aneurysm surgery under DHCA is significantly higher than in ordinary aneurysm surgery. The largest published series [9] reports a perioperative mortality of $14 \%$ and a combined rate of permanent treatment-related morbidity and mortality of $32 \%$. This recent study, however, reported a contemporary population of patients harbouring complex aneurysms not lending themselves to one of the aforementioned alternative treatment forms. In view of the otherwise unfavourable natural course of these aneurysms, the reported $63 \%$ good outcome rate justifies the procedure in a small subset of highly selected patients. For the time being, DHCA should be considered a procedure of last resort for otherwise untreatable aneurysms.
Intra-ischaemic mild hypothermia in focal cerebral ischaemia: The situation of temporary parent artery clipping during cerebral aneurysm surgery closely resembles animal models of transient focal ischaemia, wherein intra-ischaemic hypothermia was more effective than delayed post-ischaemic cooling and was more effective in transient ischaemia and reperfusion models [17-23]. The IHAST trial included 1,001 good grade (WFNS grades I to III) subarachnoid haemorrhage (SAH) patients undergoing aneurysm surgery and randomised them to intraoperative normothermia $\left(36.5^{\circ} \mathrm{C}\right)$ versus mild intraoperative hypothermia $\left(33^{\circ} \mathrm{C}\right)$. Surprisingly, intraoperative hypothermia did not improve neurologic outcome [24]. Theoretically, the group randomised to mild hypothermia should have enjoyed the cerebroprotective effects of mild intra-ischaemic hypothermia intraoperatively, especially during the ischaemic challenge of temporary clipping. It was subsequently speculated that possibly not all IHAST patients had experienced a significant intraoperative ischaemic challenge and in turn a subgroup analysis of those 441 patients who indeed required temporary clipping was performed [25]. Among these patients, the duration of focal ischaemia - that is, temporary clipping - was the most important determinant of outcome, with those patients requiring temporary clipping of $>20$ minutes faring significantly worse. Even in this selected subpopulation, mild intra-ischaemic hypothermia did not significantly alter neurologic outcome after intraoperative focal ischaemia. The IHAST results raise the question of how to document relevant intraoperative focal ischaemic challenges and the beneficial effects of neuroprotective interventions in future studies. Even after $\mathrm{SAH}$, gentle surgical manipulation and brief temporary clipping in expert hands were apparently not adequate ischaemic challenges to resemble animal models of temporary focal cerebral ischaemia and reperfusion.

\section{References}

1. Fay T: Early experiences with local and generalized refrigeration of the human brain. J Neurosurg 1959, 16:239-260.

2. Illievich UM, Spiess CK: Hypothermic therapy for the injured brain. Curr Opin Anaesth 1994, 7:394-400.

3. Busto R, Dietrich WD, Globus MY, Valdes I, Scheinberg P, Ginsberg MD: Small differences in intraischemic brain temperature critically determine the extent of ischemic neuronal injury. J Cereb Blood Flow Metab 1987, 7:729-738.

4. Holzer M, Bernard SA, Hachimi-Idrissi S, Roine RO, Sterz F, Müllner M, Collaborative Group on Induced Hypothermia for Neuroprotection after Cardiac Arrest: Hypothermia for neuroprotection after cardiac arrest: systematic review and individual patient data meta-analysis. Crit Care Med 2005, 33:414-418.

5. Shankaran S, Laptook AR, Poole WK: Whole body hypothermia for neonates with hypoxic ischemic encephalopathy. N Engl J Med 2005, 353:1574-1584.

6. Stein LH, Elefteriades JA: Protecting the brain during aortic artery surgery: an enduring debate with unanswered questions. I Cardiothorac Vasc Anesth 2010, 24:316-321.

7. Minatoya K, Ogino H, Matsuda H: Evolving selective cerebral perfusion for aortic arch replacement: high flow rate with moderate hypothermic circulattory arrest. Ann Thorac Surg 2008, 86:1827-1831.

8. Mack WJ, Ducruet AF, Angevine PD, Komotar RJ, Shrebnick DB, Edwards NM, Smith CR, Heyer EJ, Monyero L, Connolly ES, Solomon RA: Deep hypothermic circulatory arrest for complex cerebral aneurysms: lessons learned. Neurosurgery 2007, 60:815-827.

9. Ponce FA, Spetzler RF, Han PP, Wait SD, Killory BD, Nakaji P, Zabramski JM: Cardiac standstill for cerebral aneurysms in 103 patients: an update on the experience at the Barrow Neurological Institute. J Neurosurg 2011, 114:877-884.

10. Schebesch KM, Proescholdt M, Ullrich OW, Camboni D, Moritz S, Wiesenack C, Brawanski A: Circulatory arrest and deep hypothermia for the treatment of complex intracranial aneurysms - results from a single European center. Acta Neurochir (Wien) 2010, 152:783-792.

11. Spetzler RF, Hadley MN, Rigamonti D, Carter LP, Raudzens PA, Shedd SA Wilkinson E: Aneurysms of the basilar artery treated with circulatory arrest, hypothermia, and barbiturate cerebral protection. J Neurosurg 1988, 68:868-879.

12. Yamada M, Nishikawa F, et al: Anesthetic management for clipping a giant basilar artery aneurysm with moderate hypothermia, extracorporeal circulation assistance, and propofol infusion. J Neurosurg Anesthesiol 2003, 15:274-277.

13. Szikora I, Berentei Z, Kulcsar Z, Marosfoi M, Vajda ZS, Lee W, Berez A, Nelson PK: Treatment of intracranial aneurysms by functional 
reconstruction of the parent artery: the Budapest experience with the pipeline embolization device. AJNR Am J Neuroradiol 2010, 31:1139-1147.

14. Sughrue ME, Saloner D, Rayz VL, Lawton MT: Giant intracranial aneurysms: evolution of management in a contemporary surgical series. Neurosurgery 2011, 69:1261-1271.

15. Bendok BR, Gupta DK, Dahme EJ, Eddleman CS, Adel JG, Sherma AK, Surdell DL, Bebawy JF, Koht A, Batjer HH: Adenosine for temporary flow arrest during intracranial aneurysm surgery: a single center retrospective review. Neurosurgery 2011, 69:815-821.

16. Lownie SP, Menkis AH, Craen RA, Mezon B, MacDonald J, Steinman DA: Extracorporeal femoral to carotid artery perfusion in selective brain cooling for a giant aneurysm. J Neurosurg 2004, 100:343-347.

17. Andres RH, Steinberg GK, Guzman R: Intraoperative hypothermia during vascular neurosurgical procedures. Neurosurg Focus 2009, 26:1-6.

18. Mackensen GB, McDonagh DL, Warner DS: Perioperative hypothermia: use and therapeutic implications. J Neurotrauma 2009, 26:342-358.

19. Huh PW, Belayev L, Zhao W, Koch S, Busto R, Ginsberg MD: Comparative neuroprotective effects of prolonged moderate intraischemic and postischemic hypothermia in focal cerebral ischemia. J Neurosurg 2000, 92:91-99.

20. Baker CJ, Fiore AJ, Frazzini VI, Choudhri TF, Zubay GP, Solomon RA: Intraischemic hypothermia decreases the release of glutamate in the cores of permanent focal cerebral infarcts. Neurosurgery 1995, 36:994-1002.

21. Kader A, Brisman MH, Maraire N, Huh JT, Solomon RA: The effect of mild hypothermia on permanent focal ischemia in the rat. Neurosurgery 1992, 31:1056-1061.

22. Goto Y, Kassell NF, Hiramatsu KI, Soleau SW, Lee KS: Effects of intraischemic hypothermia on cerebral damage in a model of reversible focal ischemia. Neurosurgery 1993, 32:980-985.

23. Maier CM, Sun GH, Kunis D, Yenari MA, Steinberg GK: Delayed induction and long term effects of mild hypothermia in a focal model of transient cerebral ischemia: neurological outcome and infarct size. J Neurosurg 2001, 94:90-96.

24. Todd MM, Hindman BJ, Clarke WR, Torner JC, Intraoperative Hypothermia for Aneurysm Surgery Trial: Mild intraoperative hypothermia during surgery for intracranial aneurysms. N Engl J Med 2005, 352:135-145.

25. Hindman BJ, Bayman EO, Pfisterer W, Torner JC, Todd MM, IHAST investigators: No association between intraoperative hypothermia or supplemental protective drug and neurologic outcomes in patients undergoing temporary clipping during cerebral aneurysm surgery. Anesthesiology 2010, 112:86-101.

\section{A18}

Temperature management in central nervous infection

Erich Schmutzhard*, Peter Lackner, Ronny Beer, Marlene Fischer,

Anelia Dietmann, Bettina Pfausler

Department of Neurology, Neurocritical Care Unit, Medical University

Hospital Innsbruck, Austria

Critical Care 2012, 16(Suppl 2):A18

Introduction: Brian Kellock quotes in his book The Fibreman the sevenyear-old (that is, in the year 1918) Denis Burkitt discussing respectfully his uncle Dr Roland Burkitt's legendary reputation amongst his patients in Kenya, Africa, as: '... he believed in curing fevers, in particular in delirious patients, by artificially lowering the patients' temperature ...' [1].

Obviously, Dr Roland Burkitt, the uncle of the legendary Prof. Dr Denis Burkitt, after whom the African lymphoma and its association with EpsteinBarr virus was named, had realized that lowering the body temperature in patients with high fevers, in Kenya highly likely to being due to cerebral malaria, meningitis or encephalitis, might influence positively the course of such potentially - at this time, in many instances, definitely - lifethreatening disease [1]. Zdravev stipulated that otogenous brain abscesses not only need to be surgically evacuated but, when causing cerebral herniation, may benefit from lowering body temperature. He was the first to conclude that high body temperature in patients with increased intracranial pressure may be a deleterious association [2].

The wide variety of mechanisms of injury that are exaggerated by hyperthermia and may be ameliorated by moderate hypothermia are described elsewhere in this supplement (see abstract $A 1$ ). They include mechanisms of neuroexcitotoxicity [3], release of free radicals, changes in blood-brain barrier and vascular permeability, the release of proinflammatory mediators, drawing leucocytes across the blood-brain barrier, increasing the number of inflammatory cells in the brain tissue and the passage of neutrophils, phagocytes, monocytes and macrophages into the brain, additionally injuring neuronal cells by stimulating further immune reactions [4]. Whether interfering with these mechanisms by moderate hypothermia may reduce secondary insult onto neuronal cells and brain tissue is still a matter of discussion. What has been known for long time is that increased intracranial pressure, as frequently seen in viral encephalitis, severe bacterial meningitis and/or brain abscesses, may be modified by therapeutic hypothermia/targeted temperature management [5]. In addition, long-term morbidity and mortality in CNS infection may additionally deteriorate if bacterial meningitis is complicated by cerebral infarction, occurring during the course of disease [6]. Thus, preventing cerebral infarction may be an important tool in reducing both morbidity and mortality in adults with community-acquired bacterial meningitis.

Prandini and colleagues have shown that mild hypothermia reduces remarkably polymorphonuclear leucocyte infiltration in induced brain inflammation in rats compared with those without hypothermia [4].

After any type of brain injury, in particular, ischemic and ischemia reperfusion injury, the reduced brain oxygen supply quickly leads to a decrease in ATP and phosphocreatine levels, thus initiating a complex cascade of events involving excessive calcium influx into brain cells, excessive glutamate receptor activation and neuronal hyperexcitability; that is, triggering off the excitotoxic cascade [3]. Exactly this excitotoxic cascade may be responsible for provoking overt or subtle epileptic seizures in patients with CNS infection, thereby deteriorating the prognosis of these patients. Irazuzta and colleagues could nicely show that in rabbits hypothermia decreases excitatory neurotransmitter release in bacterial meningitis, in particular, the release of glutamate and aspartate, known to be involved in the pathogenesis of neuronal injury in meningitis, suggesting that hypothermia may attenuate excess neuronal stress in this disease [3].

Febrile refractory status epilepticus may be caused by presumed encephalitis carrying a very high morbidity and mortality rate [7]. In addition, it is believed that hyperthermia aggravates brain damage due to continuing epileptic activity. In a retrospective analysis Nakagawa and colleagues found a significant improvement of outcome in children with such febrile refractory status epilepticus who were treated by therapeutic hypothermia with subsequent prophylactic normothermia compared with those children with the same disease but treated only by conventional therapy without fever management [7]. The authors conclude that treatment with therapeutic hypothermia plus subsequent prophylactic normothermia may reduce neurological damage in such patients with severe acute encephalopathy due to refractory febrile convulsive status epilepticus. Another Japanese group treated an adult patient with influenza type $A$ virus-associated encephalopathy with routine therapeutics as oseltamivir and methylprednisolone pulse therapy. Because of severe brain swelling, hypothermia was added. This combination treatment led to full recovery without neurologic sequelae [8]. A similar observation has been reported in 43 children with acute inflammatory encephalopathy and/or encephalitis, out of whom 27 were treated with mild hypothermia. These 27 children were compared with 16 similar patients who were cared for at normothermia levels [9]. The effect of therapeutic hypothermia for children with acute encephalopathy/encephalitis was observed to be dependent on the timing when cooling was initiated. Early cooling (within 12 hours of initial neurological signs of encephalopathy/encephalitis) improved outcome whereas delayed cooling (after 12 hours) tended to be even deleterious [9].

In adults, therapeutic hypothermia in CNS infection has been limited to single case reports and case series. Cuthbertson and colleagues reported induced hypothermia, together with barbiturate coma, in a meningitis patient associated with intractable raised intracranial pressure. Throughout the entire period of mild hypothermia $\left(35^{\circ} \mathrm{C}\right)$, achieved by thiopental and external cooling, cerebral perfusion pressure was maintained above $70 \mathrm{mmHg}$. Barbiturate-induced coma and induced hypothermia were continued for 48 hours when complications (pancreatitis, ventilatorassociated pneumonia) prompted one to stop these two therapy modalities. However, the patient remained without any ICP rebound, eventually being weaned from the ventilator and making a rather uneventful recovery, being discharged home with reportedly no neurologic deficits 3 weeks after the disease had began [5]. Similarly, a case with space occupying herpes 
simplex virus encephalitis has been reported by Wagner and colleagues. The authors correctly state that conventional intracranial pressure-lowering modalities are limited in such severe herpes simplex virus encephalitis patients and more aggressive treatment options are needed. They induced moderate hypothermia $\left(33^{\circ} \mathrm{C}\right)$, resulting in fast and sustained control of intracranial pressure. Eventually the patient regained a relatively good functional outcome, being able to walk unassisted (GOS 4). It needs to be noted that rewarming was done very slowly $\left(1^{\circ} \mathrm{C} /\right.$ day $)$; that is, over a period of 4 days [10].

Very recently two larger cases series have been published on the use of therapeutic hypothermia both in adult viral meningoencephalitis and in adult community-acquired meningitis by the same Croatian group:

Therapeutic hypothermia in adult viral meningoencephalitis [11]: Eleven adult patients with viral or presumed viral encephalitis (two TBE, two HSV, one VZV, six unknown) pathogen were treated with mild hypothermia, rectal temperature aimed to be 32 to $34^{\circ} \mathrm{C}$. The authors, however, do not elaborate on rewarming, speed of rewarming and any adverse effects of rewarming. Remarkably only one patient $(1 / 11=9 \%)$ died, $3 / 11$ recovered fully, 2/11 with only minor impairment and $5 / 11$ with moderate to severe residual neurological deficit. All patients showed severely impaired GCS at admission (median GCS 8 (range 3 to 10)) and median Acute Physiology and Chronic Health Evaluation score (APACHE) being 24 (range 12 to 32). A major drawback of this study is the lack of ICP monitoring, sonographic measurement of optic nerve sheath diameter having been used as surrogate markers for ICP, both methods not being as reliable as continuous ICP and CPP monitoring. The mortality rate of $9 \%$ in this case series compared with $29 \%$ in patients with viral meningoencephalitis treated in their unit before implementation of the therapeutic hypothermia protocol. The authors suggest that in carefully selected patients, carefully regarding vasoreactivity status, and, of course, only in those with initially reduced cerebral perfusion pressure, therapeutic hypothermia may be a tool to reduce long-term morbidity and mortality.

Therapeutic hypothermia in bacterial meningitis [12]: The same Croatian group presented a series of 10 patients with severe bacterial meningitis (nine patients: pneumococci, one patient: Escherichia coli) with an initial median GCS of 6 (range 3 to 9), APACHE II ranging from 22 to 34 (median 31). They employed the same protocol of non-invasive ICP monitoring (using transcranial Doppler sonography, optic nerve sheath diameter sonography and jugular bulb oximetry). Hypothermia was induced by intravenous infusion of cold isotonic saline and maintained with continuous venovenous hemofiltration at 32 to $34^{\circ} \mathrm{C}$. Nothing is said about rewarming, rewarming speed and duration of hypothermia. Two patients died within 48 hours from admission because of refractory intracranial hypertension, two more patients with severe residual neurological deficits (GOS 2) died later on, after discharge from the ICU, because of late-onset nosocomial sepsis; in total, a rather high mortality rate $[13,14]$. The surviving six patients had a mean ICU stay of 22 days (range 8 to 36), two had a severe and two a moderate residual neurologic deficit. Two of the entire group of 10 patients with bacterial meningitis had a complete neurological recovery (GOS 5).

The authors discuss hypothermia as a neuroprotective measure in severe bacterial meningitis. In nine (out of 10) patients pneumococci were isolated as the causative organisms; however, the authors do not elaborate whether these patients - qualifying early for dexamethasone treatment, in particular, since they showed severe clinical signs and symptoms of increased intracranial pressure - had been given dexamethasone or not. They discuss that corticosteroids have not substantially changed disease outcomes among patients with most severe pneumococcal meningitis. Exactly this aspect is in contrast to the widely accepted knowledge and level of evidence respectively. Recently a meta-analysis has clearly shown that European patients with pneumococcal bacterial meningitis (aged $>55$ years) definitely benefit from the adjunctive application of dexamethasone [15]. Only one single patient would not have qualified for this definition (47-yearold female being severely immunocompromised). This might explain the high mortality rate despite the administration of the adjunctive therapy with moderate hypothermia. The authors correctly claim that any adjunctive therapy should be continued beyond the very first hours and days since it has been known that in severe bacterial meningitis vasculopathy leading to ischemic stroke may ensue even days after onset. Adequate maintenance of sufficient cerebral perfusion pressure until recovery of $\mathrm{CO}_{2}$ reactivity (not to be expected before day 4 after initiation of antibiotic treatment) is essential and requires therapeutic hypothermia to be maintained for this period of time. However, the results of this pilot trial in 10 consecutive patients do not justify one to recommend therapeutic hypothermia as adjunctive therapy in bacterial (pneumococcal) meningitis. Whether selected patients with either intractable ICP, dangerously low CPP or infection-associated refractory status epilepticus benefit from moderate hypothermia needs to be elucidated; today this is still a case to case decision.

\section{References}

1. Kellock B: The Fibreman, the Life Story of Dr. Denis Burkitt Lion Publishers; Oxford Oxfordshire OX2 7DH, UK, 1st 1985, p8.

2. Zdravev P: Treatment of cerebral hernia following surgery of an otogenous brain abscess. Ann Otolaryngol 1951, 68:201-205.

3. Irazuzta JE, Olson J, Kiefaber MP, Wong H: Hypothermia decreases excitatory neurotransmitter release in bacterial meningitis in rabbits. Brain Res 1999, 847:143-148.

4. Prandini MN, Neves Filho A, Lapa AJ, Stavale JN: Mild hypothermia reduces polymorphonuclear leukocytes infiltration in induced brain inflammation. Arq Neuropsiquiatr 2005, 63:779-784.

5. Cuthbertson BH, Dickson R, Mackenzie A: Intracranial pressure measurement, induced hypothermia and barbiturate coma in meningitis associated with intractable raised intracranial pressure. Anaesthesia 2004, 59:908-911.

6. Schut ES, Lucas MJ, Brouwer MC, et al: Cerebral infarction in adults with bacterial meningitis. Neurocrit Care 2011 in press.

7. Nakagawa T, Fujita K, Saji Y, Maruyama A, Nagase H: Induced hypothermia/ normothermia with general anesthesia prevents neurological damage in febrile refractory status epilepticus in children. No To Hattatsu 2011, 43:459-463.

8. Sakurai T, Kimura A, Tanaka Y, Hozumi I, Ogura S, Inzuka T: Case of adult influenza type A virus-associated encephalopathy successfully treated with primary multidisciplinary treatments. Rinsho Shinkeigaku 2007, 47:639-643.

9. Kawano G, Iwata O, Iwata $\mathrm{S}$, et al: Determinants of outcomes following acute child encephalopathy and encephalitis: pivotal effect of early and delayed cooling. Arch Dis Child 2011, 96:936-941.

10. Wagner I, Staykov D, Volbers B: Therapeutic hypothermia for spaceoccupying Herpes simplex virus encephalitis. Minerva Anestesiol 2011, 77:371-374.

11. Kutlesa $M$, Barsic B, Lepur D: Therapeutic hypothermia for adult viral meningoencephalitis. Neurocrit Care 2011, 15:151-155.

12. Lepur D, Kutlesa M, Barsic B: Induced hypothermia in adult communityacquired bacterial meningitis - more than just a possibility? J Infect 2011, 62:172-177.

13. Branco RG, Tasker RC: Meningococcal meningitis. Curr Treat Options Neurol 2010, 12:464-474.

14. Weisfelt M, van de Beek D, Spanjaard L, Reitsma JB, de Gans J: A risk score for unfavorable outcome in adults with bacterial meningitis. Ann Neurol 2008, 63:90-97.

15. van de Beek D, Farrar JJ, de Gans J, et al: Adjunctive dexamethasone in bacterial meningitis: a meta-analysis of individual patient data. Lancet Neurol 2010, 9:254-263.

\section{A19}

Complications of hypothermia: infections

Gregor Broessner, Marlene Fischer, Peter Lackner, Bettina Pfausler, Erich Schmutzhard

Neurologic Intensive Care Unit, Medical University Innsbruck, Austria Critical Care 2012, 16(Suppl 2):A19

Background: Therapeutic hypothermia $(\mathrm{TH})$ is a very elegant way of inducing short-term and long-term neuroprotection in various disease entities. It has become the standard of care after cardiac resuscitation with an impressive outcome improvement in prospective randomised trials. However, by a broader use of this sophisticated measure the critical care community has become aware of potential side effects limiting its effect on patient outcome. Among others, an increased rate of infections is observed under therapeutic hypothermia and controlled normothermia. The pathophysiological considerations by which TH increases infectious complications comprise reduced inflammatory response and suppression of leukocyte migration and phagocytosis. All together, these observations justify a high vigilance towards infectious manifestations if temperature modulation measures, namely therapeutic hypothermia and controlled normothermia, are used in critical care patients. 
Pathophysiological considerations about hypothermia and infections: Intriguing data derived from animal models showing a potent neuroprotective effect in various disease entities induced by hypothermia gave way to a broader use of this method in humans. Thus, therapeutic hypothermia has become the standard of care after cardiac resuscitation as studies demonstrated its strong neuroprotective effect and neurologic outcome improvement $[1,2]$. It is now recommended by the European Resuscitation Council and the International Liaison Committee on Resuscitation in cases of comatose adults with spontaneous circulation after out-of-hospital cardiac arrest (OHCA) [3]. However, in indications other than resuscitation, such promising results could not be achieved in prospective trials shifting the scientific focus on possible side effects of TH [4-6]. Rewarming injury, shivering, electrolyte dysbalance, pharmacological and pharmacodynamic alterations, cardiovascular effects including arrhythmia, insulin resistance and infections have recently been attributed as limitations occurring in a dose-dependent fashion under TH [7]. Taken together, maximal reduction of these side effects should be a treatment goal if dealing with temperature control measures irrespective of the target temperature. Today, infectious complications are thought to be one of the major contributors limiting the effects of hypothermia [7-12]. Thus advancing diagnostic approach, prevention and treatment of these infectious complications is a great concern of the scientific critical care community and need to be addressed in future prospective trials. In various studies enrolling patient populations suffering from such different diseases as traumatic brain injury, ischaemic stroke or resuscitation post cardiac arrest, an increased rate of infections under TH was observed $[4,6,11]$. Whether this negative effect has to be attributed to a specific cooling measure remains under debate; however, this hypothesis is unlikely as increased infections are found under both endovascular and surface cooling measures.

The biological interpretation of the pathophysiological backgrounds is challenging as temperature modulation inhibits various inflammatory responses on different levels that are only partly understood today $[7,8]$. Hypothermia impairs the secretion of proinflammatory cytokines and suppresses leukocyte migration and phagocytosis $[7,8]$. Recently it has been speculated that hypothermia may induce insulin resistance leading to hyperglycaemia possibly promoting infection onset $[8,13]$.

However, increased rate of infections has also been observed not only under TH but also under endovascularly controlled prophylactic normothermia in patients with severe cerebrovascular disease $[10,14]$. A significant increase of infectious complications was observed in the endovascular treatment group although TH was strictly avoided. Importantly in this study from our group, analysis of the inflammatory parameters revealed a significant increase of $\mathrm{C}$-reactive protein (CRP) in the prophylactic normothermia group whereas procalcitonin (PCT) and white blood cell count were not elevated [10]. This is a crucial point as it might indicate that temperature modulation may influence the prognostic value of inflammatory parameters [10].

In a retrospective review by Mongardon and coworkers including 421 patients being treated after cardiac arrest, in 281 patients (67\%) an infectious complication was diagnosed [11]. Pneumonia was the most frequent, followed by bloodstream infections and catheter-related infections [11]. Gram-negative bacteria were the most frequently isolated infectious germs, but the main pathogen detected was Staphylococcus aureus [11]. The high rate of reported pneumonia raises the question of whether intubation at an early stage should be considered in patients under TH to minimise the risk of aspiration.

Is this surcharge too much and how can we minimise it in clinical routine?: Hospital-acquired infections lead to secondary injury in patients and are responsible for a considerable cost increase especially in critical care patients $[15,16]$. An increased rate of infections under controlled normothermia and therapeutic hypothermia has been described in patients suffering from ischaemic stroke, traumatic brain injury, spontaneous subarachnoid haemorrhage, post resuscitation and intracerebral haemorrhage $[6,9,10]$. If this observation is associated with significantly impaired outcome or even mortality is under debate [6,9-11]. However, there is general consensus that infections lead to prolonged ICU treatment, secondary injury and lastly to cost increase with significant global economic burden [15]. Therefore, the critical care community has to find effective strategies to minimise the risk of infection complications.
Temperature modulation by any means has to be combined with a standard operation procedure including: routine microbiological surveillance including blood, urine, respiratory specimen work-up; radiological pneumonia surveillance; daily monitoring of inflammatory parameters (CRP, PCT, leukocytes); routine check of catheter insertion sites and timely catheter replacement; avoidance of hyperglycaemia (under TH) and hypoglycaemia (while rewarming!); and monitor performance of cooling device as a high cooling power/rate might indicate fever even if the body core temperature is normal or even $<36^{\circ} \mathrm{C}$. Conclusion: These observations justify a high level of vigilance towards infectious manifestations if temperature modulation measures, namely therapeutic hypothermia and controlled normothermia, are used in critical care patients. Whether, at all, the early use of antibiotics in case of suspected infections under TH is justified has to be addressed in future prospective trials.

\section{References}

1. Mild therapeutic hypothermia to improve the neurologic outcome after cardiac arrest. N Engl J Med 2002, 346:549-556.

2. Bernard SA, Gray TW, Buist MD, et al: Treatment of comatose survivors of out-of-hospital cardiac arrest with induced hypothermia. $N$ Engl J Med 2002, 346:557-563.

3. Nolan JP, Morley PT, Vanden Hoek TL, et al: Therapeutic hypothermia after cardiac arrest: an advisory statement by the advanced life support task force of the International Liaison Committee on Resuscitation. Circulation 2003, 108:118-121

4. Clifton GL, Miller ER, Choi SC, et al: Lack of effect of induction of hypothermia after acute brain injury. N Engl J Med 2001, 344:556-563.

5. Clifton $G L$, Valadka A, Zygun D, et al: Very early hypothermia induction in patients with severe brain injury (the National Acute Brain Injury Study: Hypothermia II): a randomised trial. Lancet Neurol 2011, 10:131-139.

6. Hemmen TM, Raman R, Guluma KZ, et al: Intravenous thrombolysis plus hypothermia for acute treatment of ischemic stroke (ICTUS-L): final results. Stroke 2010, 41:2265-2270.

7. Polderman KH, Herold I: Therapeutic hypothermia and controlled normothermia in the intensive care unit: practical considerations, side effects, and cooling methods. Crit Care Med 2009, 37:1101-1120.

8. Polderman $\mathrm{KH}$ : Induced hypothermia and fever control for prevention and treatment of neurological injuries. Lancet 2008, 371:1955-1969.

9. Broessner $G$, Beer R, Lackner P, et al: Prophylactic, endovascularly based, long-term normothermia in ICU patients with severe cerebrovascular disease: bicenter prospective, randomized trial. Stroke 2009, 40:e657-e665.

10. Broessner $G$, Lackner P, Fischer M, et al: Influence of prophylactic, endovascularly based normothermia on inflammation in patients with severe cerebrovascular disease: a prospective, randomized trial. Stroke 2010, 41:2969-2972.

11. Mongardon N, Perbet S, Lemiale V, et al: Infectious complications in outof-hospital cardiac arrest patients in the therapeutic hypothermia era. Crit Care Med 2011, 39:1359-1364.

12. Kamps M, Bisschops LA, van der Hoeven JG, et al: Hypothermia does not increase the risk of infection: a case control study. Crit Care 2011, 15:R48.

13. van den Berghe $G$, Wouters $P$, Weekers $F$, et al: Intensive insulin therapy in critically ill patients. N Engl J Med 2001, 345:1359-1367.

14. Broessner $G$, Lackner $P$, Hoefer $C$, et al: Influence of red blood cell transfusion on mortality and long-term functional outcome in 292 patients with spontaneous subarachnoid hemorrhage. Crit Care Med 2009, 37:1886-1892.

15. Farmer JC: Therapeutic hypothermia: is there an unintended surcharge? Crit Care Med 2011, 39:1570-1571.

16. Stone PW, Braccia D, Larson E: Systematic review of economic analyses of health care-associated infections. Am J Infect Control 2005, 33:501-509.

\section{A20}

\section{Hypothermia and coagulation}

Kees $\mathrm{H}$ Polderman

Department of Critical Care Medicine, University of Pittsburgh Medical Center, Pittsburgh, PA, USA

Critical Care 2012, 16(Suppl 2):A20

Introduction: The effects of hypothermia on coagulation may represent a two-edged sword in patients with acute brain injury who are treated with 
therapeutic cooling. On the one hand inhibition of coagulation can have positive effects, such as improvements in the microcirculation and inhibition of the formation of harmful microthrombi in the brain [1]. On the other hand this could lead to increased bleeding risk and thereby cause harm to patients, especially if they have suffered traumatic injuries or are actively bleeding for other reasons. This manuscript will briefly discuss what is known about the effects of hypothermia on cooling.

In vitro and experimental data: The effects of hypothermia on coagulation have been studied mostly in vitro. Very mild hypothermia (down to $35^{\circ} \mathrm{C}$ ) has no effect on any part of the coagulation cascade. Temperatures below $35^{\circ} \mathrm{C}$ can in some cases (but not in all patients, see below) induce mild platelet dysfunction and sometimes a mild decrease in platelet count. When temperatures drop below $33^{\circ} \mathrm{C}$ other steps in the coagulation cascade, such as the synthesis and kinetics of clotting enzymes and plasminogen activator inhibitors, can also be affected [2-8]. Recently, Ruzicka and coworkers performed a study in which they precisely measured thromboelastography in healthy subjects at a temperature range starting at $38^{\circ} \mathrm{C}$ all the way down to $12^{\circ} \mathrm{C}$ [9]. They reported that decreasing temperatures led to a progressive delay in the initiation of thrombus formation, as well as a decrease in the speed of clot creation and growth. However, significant effects of hypothermia on this parameter began only at $30^{\circ} \mathrm{C}$, progressing rapidly below this temperature but reaching statistical significance only at $24^{\circ} \mathrm{C}$ [9]. These authors also found that once clot formation had been completed, the stability of the clot could no longer be influenced by hypothermia; that is, the clots once formed remained stable regardless of temperature. Of note, there was significant interindividual variability in the response of the coagulation parameters to cooling [9].

Hanke and coworkers $[10,11]$ reported that the anticoagulatory effects of hypothermia were markedly increased if acidosis was present, that the effects of hypothermia could be effectively reversed by administering DDAVP and fibrinogen, but that these drugs worked well only if acidosis was corrected [10].

Finally, a number of animal studies have looked at the effects of hypothermia on hematoma formation in models for intracranial hemorrhage and subdural hematoma [12-16]. These studies have not found any evidence for increased hematoma growth or bleeding risk associated with mild hypothermia; in fact, the opposite effect (decreased hematoma volume and vascular brain edema) was observed in most of these studies [12-16]. Indeed a randomized clinical trial is currently being organized to test the safety and efficacy of cooling in patients with intracranial hemorrhage [17].

Clinical studies: The clinical effects of mild hypothermia on bleeding appear to be minor, and clinical studies suggest that the risk of severe bleeding associated with mild hypothermia is very low or even absent. None of the large studies in cardiac arrest, stroke, or traumatic brain injury have reported significant increase in bleeding risks associated with therapeutic cooling, although it should be emphasized that actively bleeding patients were excluded from these studies [12].

Preliminary data suggest that hypothermia can even be used safely in combination with thrombolytic therapy. Hemmen and colleagues performed a prospective controlled clinical trial in 58 patients with acute ischemic stroke, 28 of whom were treated with hypothermia $\left(33^{\circ} \mathrm{C}\right)$ combined with thrombolytic therapy [18]; they found that the risk of hemorrhagic conversion did not increase in patients treated with both hypothermia and TPA compared to those treated with TPA alone, and in fact the risk of symptomatic ICH trended to be lower in cooled patients [18].

Spiel and colleagues studied the effect of hypothermia induced by cold fluid infusion in cardiac arrest patients; they reported only slightly prolonged clotting time as measured by rotation thrombelastography [19].

Storm and coworkers compared risk and severity of bleeding in cardiac arrest patients treated with mild hypothermia and thrombolysis to matched historical patients treated with thrombolysis only [20]. They found that the incidence of bleeding was not increased by hypothermia, although there was a trend towards more red blood cell units being required to reach target hematocrit in hypothermic patients who did develop bleeding complications. As neurological outcomes were significantly better in patients treated with both thrombolytics and hypothermia, even in those who developed bleeding complications, the authors concluded that bleeding risks should not be viewed as a reason to withhold hypothermia treatment [20].

Tuma and colleagues performed a retrospective analysis from a trauma registry database and identified patients who had developed cardiac arrest and had been treated with hypothermia [21]. The number of patients was small but they found no increased complication rate from hypothermia, in particular bleeding, in their patients. This is noteworthy as hypothermia has gained a negative reputation among those treating multi-traumatized patients, and is seen as one of the factors in the 'lethal triad' of shock, acidosis and hypothermia [22]. The discrepancy may be explained by the interplay between acidosis and hypothermia, as explained above; the effects of (deep) hypothermia on coagulation are pronounced, and difficult to reverse, mainly if there is simultaneous acidosis [22]. However, in the absence of acidosis the effects of hypothermia are much less pronounced, and far more easily controllable and reversible. Most patients undergoing elective treatment with mild hypothermia will not have severe acidosis, and therefore effects of hypothermia on coagulation will be minimal.

These clinical data and the physiological effects of cooling should be taken into account when decisions are made on whether or not to use hypothermia in patients who are actively bleeding, or who are at high risk for bleeding. If possible the (potential) source of bleeding should be (surgically) controlled before cooling is initiated. If this is not possible the risks of bleeding should be weighed against the benefits of cooling, and careful consideration should be given to the depth of hypothermia induced in that patient. Because very mild hypothermia $\left(35^{\circ} \mathrm{C}\right)$ does not affect coagulation in any patient this temperature can be safely induced even in patients with very high bleeding risk. At temperatures below $35^{\circ} \mathrm{C}$ some patients may begin to have mild platelet dysfunction (although in most patients this will occur only at lower temperatures). These effects will be significantly magnified in the presence of acidosis.

In my hospital patients who are admitted after cardiac arrest will usually be cooled to 32 or $33^{\circ} \mathrm{C}$ for a period of 24 hours, followed by slow rewarming over 16 to 24 hours. If such a patient has concomitant severe traumatic injury (for example, blunt abdominal trauma) and/or has injuries that pose a high bleeding risk that cannot easily be surgically controlled (for example, liver laceration), this patient typically would be cooled to $35^{\circ} \mathrm{C}$, and cooling would be continued for 48 or 72 hours instead of our current standard of 24 hours. Similarly, if a patient develops severe uncontrollable bleeding during hypothermia therapy (for example, major upper Gl bleed with hemodynamic instability) we would consider rewarming this patient to $35^{\circ} \mathrm{C}$ (or maintaining $33^{\circ} \mathrm{C}$ and giving platelet transfusion). In mildly elevated bleeding risk we will cool the patient to $33^{\circ} \mathrm{C}$ and will treat with platelet transfusion and DDAVP if bleeding complications occur (unless there are severe counter-indications such as recent stent placement).

Of note, it is unknown whether decreasing the intensity of cooling while extending the duration of therapy will provide similar benefits as applying treatment according to published guidelines [23]. Therefore, of course those not at high bleeding risk should be cooled to between 32 and $34^{\circ} \mathrm{C}$ as current guidelines recommend [23].

Conclusion: Although mild to moderate hypothermia has some effect on the coagulation system the clinical risk of bleeding associated with cooling appears to be very low. This will, however, increase significantly if the patient has moderate-to-severe acidosis. No effects of hypothermia on coagulation occur in any patient as long as temperature is $\geq 35^{\circ} \mathrm{C}$, and patients at very high bleeding risk can safely be cooled to this temperature. References

1. Polderman $\mathrm{KH}:$ Mechanisms of action, physiological effects, and complications of hypothermia. Crit Care Med 2009, 37:S186-\$202.

2. Michelson AD, MacGregor H, Barnard MR, Kestin AS, Rohrer MJ, Valeri CR: Hypothermia-induced reversible platelet dysfunction. Thromb Haemost 1994, 71:633-640.

3. Watts DD, Trask A, Soeken K, Perdue P, Dols S, Kaufmann C: Hypothermic coagulopathy in trauma: effect of varying levels of hypothermia on enzyme speed, platelet function, and fibrinolytic activity. J Trauma 1998, 44:846-854.

4. Valeri CR, MacGregor H, Cassidy G, Tinney R, Pompei F: Effects of temperature on bleeding time and clotting time in normal male and female volunteers. Crit Care Med 1995, 23:698-704.

5. Patt A, McCroskey B, Moore E: Hypothermia-induced coagulopathies in trauma [review]. Surg Clin North Am 1988, 68:775-785. 
6. Ferrara A, MacArthur JD, Wright HK, Modlin IM, McMillen MA: Hypothermia and acidosis worsen coagulopathy in the patients requiring massive transfusion. Am J Surg 1990, 160:515-518.

7. Reed RL, Bracey AW, Hudson JD, Miller TA, Fischer RP: Hypothermia and blood coagulation: dissociation between enzyme activity and clotting factor levels. Circ Shock 1990, 32:141-152.

8. Valeri CR, Feingold H, Cassidy G, Ragno G, Khuri S, Altschule MD: Hypothermiainduced reversible platelet dysfunction. Ann Surg 1987, 205:175-181.

9. Ruzicka J, Stengl M, Bolek L, Benes J, Matejovic M, Krouzecky A: Hypothermic anticoagulation: testing individual responses to graded severe hypothermia with thromboelastography. Blood Coagul Fibrinolysis 2012 in press, doi: 10.1097/MBC.0b013e328351885a.

10. Hanke AA, Dellweg C, Schöchl H, Weber CF, Jüttner $B$, Johanning $K$, Görlinger K, Rahe-Meyer N, Kienbaum P: Potential of whole blood coagulation reconstitution by desmopressin and fibrinogen under conditions of hypothermia and acidosis - an in vitro study using rotation thrombelastometry. Scand J Clin Lab Invest 2011, 71:292-298.

11. Hanke AA, Dellweg C, Kienbaum P, Weber CF, Görlinger K, Rahe-Meyer N: Effects of desmopressin on platelet function under conditions of hypothermia and acidosis: an in vitro study using multiple electrode aggregometry. Anaesthesia 2010, 65:688-691.

12. Polderman $\mathrm{KH}$ : Induced hypothermia and fever control for prevention and treatment of neurological injuries. Lancet 2008, 371:1955-1969.

13. Yao C, Wei G, Lu XC, Yang W, Tortella FC, Dave JR: Selective brain cooling in rats ameliorates intracerebral hemorrhage and edema caused by penetrating brain injury: possible involvement of heme oxygenase-1 expression. J Neurotrauma 2011, 28:1237-1245.

14. Kawanishi M, Kawai N, Nakamura T, Luo C, Tamiya T, Nagao S: Effect of delayed mild brain hypothermia on edema formation after intracerebral hemorrhage in rats. J Stroke Cerebrovasc Dis 2008, 17:187-195.

15. Okauchi M, Kawai N, Nakamura T, Kawanishi M, Nagao S: Effects of mild hypothermia and alkalizing agents on brain injuries in rats with acute subdural hematomas. J Neurotrauma 2002, 19:741-751.

16. Kawai N, Nakamura T, Okauchi M, Nagao S: Effects of hypothermia on intracranial hemodynamics and ischemic brain damage-studies in the rat acute subdural hematoma model. Acta Neurochir Supp/ 2000, 76:529-533.

17. Kollmar R, Juettler E, Huttner HB, Dörfler A, Staykov D, Kallmuenzer B, Schmutzhard E, Schwab S, Broessner G, CINCH investigators: Cooling in intracerebral hemorrhage (CINCH) trial: protocol of a randomized German-Austrian clinical trial. Int J Stroke 2012, 7:168-172.

18. Hemmen TM, Raman R, Guluma KZ, Meyer BC, Gomes JA, Cruz-Flores S, Wijman CA, Rapp KS, Grotta JC, Lyden PD, ICTUS-L Investigators: Intravenous thrombolysis plus hypothermia for acute treatment of ischemic stroke (ICTuS-L): final results. Stroke 2010, 41:2265-2270.

19. Spiel AO, Kliegel A, Janata A, Uray T, Mayr FB, Laggner AN, Jilma B, Sterz F: Hemostasis in cardiac arrest patients treated with mild hypothermia initiated by cold fluids. Resuscitation 2009, 80:762-765.

20. Storm C, Schefold JC, Kerner T, Schmidbauer W, Gloza J, Krueger A, Jörres A, Hasper D: Prehospital cooling with hypothermia caps (PreCoCa): a feasibility study. Clin Res Cardiol 2008, 97:768-772.

21. Tuma MA, Stansbury LG, Stein DM, McQuillan KA, Scalea TM: Induced hypothermia after cardiac arrest in trauma patients: a case series. J Trauma 2011, 71:1524-1527.

22. Martini WZ: Coagulopathy by hypothermia and acidosis: mechanisms of thrombin generation and fibrinogen availability. J Trauma 2009, 67:202-208.

23. Peberdy MA, Callaway CW, Neumar RW, Geocadin RG, Zimmerman JL, Donnino M, Gabrielli A, Silvers SM, Zaritsky AL, Merchant R, Vanden Hoek TL, Kronick SL: Part 9: post-cardiac arrest care: 2010 American Heart Association Guidelines for Cardiopulmonary Resuscitation and Emergency Cardiovascular Care. Circulation 2010, 122:S768-S786.

\section{COOLING IN CARDIOLOGY}

\section{A21}

Hypothermia in cardiogenic shock

Jesper van der Pals

Department of Cardiology, Lund University, Skane University Hospital,

Sweden

Critical Care 2012, 16(Suppl 2):A21
Cardiogenic shock is a state of inadequate systemic tissue perfusion, despite adequate left ventricular filling pressure. It is caused by extensive myocardial damage and appears to be aggravated by a systemic inflammatory response [1-4]. The result is hypotension with metabolic acidosis and often a fatal outcome. The condition affects approximately $5 \%$ of the patients with myocardial infarction, and carries a dismal prognosis if it prevails after reperfusion.

Therapeutic hypothermia has several properties of potential benefit in cardiogenic shock: Experiments with isolated myofibrils, papillary muscles and cross-circulated hearts have demonstrated that mild hypothermia increases myocardial contractility [5-7]. In the in vivo heart, mild hypothermia has been found to increase stroke volume and cardiac output $[6,8]$.

The increase in contractility is considered to be mediated by an increased myofilament sensitivity to existing $\mathrm{Ca}^{2+}$, without a corresponding increase in myocardial oxygen consumption [9]. Moreover, hypothermia reduces the metabolic rate with 5 to $7 \% /{ }^{\circ} \mathrm{C}[10,11]$, thereby reducing the demand on the circulation from the peripheral tissues. In an experimental setting, it also has the ability to reduce infarct size if applied prior to reperfusion $[12,13]$

In dog-based and porcine-based models of cardiogenic shock secondary to ischemia, therapeutic hypothermia has improved hemodynamic and metabolic parameters, and reduced mortality $[14,15]$. No randomized controlled trials of therapeutic hypothermia in cardiogenic shock in humans exist, but case series indicate that the effects observed in animal experiments can be reproduced [16-19].

In conclusion, therapeutic hypothermia is a promising treatment option for patients in cardiogenic shock that warrants further investigation.

\section{References}

1. Hochman JS: Cardiogenic shock complicating acute myocardial infarction: expanding the paradigm. Circulation 2003, 107:2998-3002.

2. Kaluski E, Hendler A, Blatt A, Uriel N: Nitric oxide synthase inhibitors in post-myocardial infarction cardiogenic shock - an update. Clin Cardiol 2006, 29:482-488.

3. Cotter G, Berger PB: Cardiogenic shock - beyond the large infarction. Crit Care Med 2006, 34:2234-2235.

4. Geppert A, Dorninger A, Delle-Karth G, Zorn G, Heinz G, Huber K: Plasma concentrations of interleukin-6, organ failure, vasopressor support, and successful coronary revascularization in predicting 30-day mortality of patients with cardiogenic shock complicating acute myocardial infarction. Crit Care Med 2006, 34:2035-2042.

5. Bjornstad $\mathrm{H}$, Tande PM, Refsum H: Mechanisms for hypothermia-induced increase in contractile force studied by mechanical restitution and postrest contractions in guinea-pig papillary muscle. Acta Physiol Scand 1993, 148:253-264.

6. Weisser J, Martin J, Bisping E, Maier LS, Beyersdorf F, Hasenfuss G, Pieske B: Influence of mild hypothermia on myocardial contractility and circulatory function. Basic Res Cardiol 2001, 96:198-205.

7. Mattheussen M, Mubagwa K, Van Aken H, Wusten R, Boutros A, Flameng W: Interaction of heart rate and hypothermia on global myocardial contraction of the isolated rabbit heart. Anesth Analg 1996, 82:975-981.

8. Nishimura $Y$, Naito $Y$, Nishioka T, Okamura $Y$ : The effects of cardiac cooling under surface-induced hypothermia on the cardiac function in the in situ heart. Interact Cardiovasc Thorac Surg 2005, 4:101-105.

9. Suga $H$, Goto $Y$, Igarashi $Y$, Yasumura $Y$, Nozawa T, Futaki S, Tanaka N: Cardiac cooling increases $\mathrm{E}_{\max }$ without affecting relation between $\mathrm{O}_{2}$ consumption and systolic pressure-volume area in dog left ventricle. Circ Res 1988, 63:61-71.

10. Polderman $\mathrm{KH}$ : Mechanisms of action, physiological effects, and complications of hypothermia. Critical Care Med 2009, 37:S186-S202.

11. Polderman $\mathrm{KH}$ : Application of therapeutic hypothermia in the intensive care unit. Opportunities and pitfalls of a promising treatment modality Part 2: practical aspects and side effects. Intensive Care Med 2004, 30:757-769.

12. Gotberg M, Olivecrona GK, Koul S, Carlsson M, Engblom H, Ugander M, van der Pals J, Algotsson L, Arheden H, Erlinge D: A pilot study of rapid cooling by cold saline and endovascular cooling before reperfusion in patients with ST-elevation myocardial infarction. Circ Cardiovasc Interv 2010, 3:400-407.

13. Götberg M M, Olivecrona GK, Engblom H, Ugander M, van der Pals J, Heiberg E, Arheden H, Erlinge D: Rapid short-duration hypothermia with cold saline and endovascular cooling before reperfusion reduces microvascular obstruction and myocardial infarct size. BMC CardiovasC Disord 2008, 8:7. 
14. Gotberg M, van der Pals J, Olivecrona GK, Koul S, Erlinge D: Mild hypothermia reduces acute mortality and improves hemodynamic outcome in a cardiogenic shock pig model. Resuscitation 2010, 81:1190-1196.

15. Boyer NH, Gerstein MM: Induced hypothermia in dogs with acute myocardial infarction and shock. J Thorac Cardiovasc Surg 1977, 74:286-294.

16. Skulec R, Kovarnik T, Dostalova G, Kolar J, Linhart A: Induction of mild hypothermia in cardiac arrest survivors presenting with cardiogenic shock syndrome. Acta Anaesthesiol Scand 2008, 52:188-194.

17. Hovdenes J, Laake JH, Aaberge L, Haugaa H, Bugge JF: Therapeutic hypothermia after out-of-hospital cardiac arrest: experiences with patients treated with percutaneous coronary intervention and cardiogenic shock. Acta Anaesthesiol Scand 2007, 51:137-142.

18. Yahagi N, Kumon K, Watanabe $Y$, Tanigami $H$, Haruna M, Hayashi $H$, Imanaka H, Takeuchi M, Ohashi Y, Takamoto S: Value of mild hypothermia in patients who have severe circulatory insufficiency even after intraaortic balloon pump. J Clin Anesth 1998, 10:120-125.

19. Moriyama $Y$, Iguro $Y$, Shimokawa S, Saigenji H, Toyohira H, Taira A: Successful application of hypothermia combined with intra-aortic balloon pump support to low-cardiac-output state after open heart surgery. Angiology 1996, 47:595-599.

\section{A22}

\section{Reperfusion injury in acute myocardial infarction}

Gregor Simonis", Ruth H Strasser, Bernd Ebner

Department of Medicine/Cardiology, Heart Center, Dresden University of Technology, Dresden, Germany

Critical Care 2012, 16(Suppl 2):A22

Background: For many years it has been shown that the size of a myocardial infarction is not only determined by ischemic damage, but also by reperfusion itself. This reperfusion injury contributes to up to $50 \%$ of the final infarct size. Mechanical postconditioning using short periods of ischemia immediately after reperfusion, pharmacologic postconditioning targeted to prevent opening of the mitochondrial permeability transition pore, and hypothermia have been shown to prevent reperfusion injury in animals and to reduce infarct size in smaller studies. Larger studies targeted to reduce heart failure and mortality are eagerly awaited.

Introduction: Since the early 1970s, infarct size has been identified as a major predictor of prognosis after myocardial infarction: infarct size parallels with arrhythmia, heart failure, and mortality [1]. Apart from this finding, infarct size is not only determined by the area at risk; that is, the myocardium perfused by the infarct-related artery. In a seminal work, Reimer and coworkers could show that irreversible myocardial injury, as determined by cardiac myocyte necrosis, progresses as a wavefront from the subendocardium towards the subepicardium [2]. This observation led to the concept that early reperfusion therapy can salvage myocardium at risk from injury. Early reperfusion therapy within the first 3 to 6 hours after the onset of ischemia was, in consequence, rapidly introduced into clinical routine and is now standard treatment of patients with acute myocardial infarction.

In parallel, it was recognized that reperfusion of temporarily nonperfused myocardium itself has effects on cellular integrity [2]. Reperfusion saves viable myocytes. However, it accelerates the disruption of irreversibly injured myocytes, and thereby permits the process of inflammation, phagocytosis, and infarct repair to begin quickly. It leads to interstitial hemorrhage from vessels that are injured by ischemia but are reperfusable at the time of reflow; that is, in the border zone of the infarction. The hemorrhage itself increases the interstitial pressure, which in consequence worsens the tissue perfusion again. Moreover, reperfusion induces severe morphologic alterations of the myocardium such as cardiac myocytes swelling, mitochondrial damage, hypercontracture, and loss of myofibrillar organization [3]. The observation that reperfusion itself influences infarct size has led to the concept of lethal reperfusion injury $[2,3]$. It became quickly clear that reperfusion injury is not only determined by mechanical factors such as hemorrhage or interstitial pressure. Reperfusion leads to the activation of many signaling pathways that contribute independently to both apoptotic and necrotic tissue injury and thus decrease the amount of viable myocardium (reviewed in $[4,5])$. The concept of additional myocardial damage is induced by lethal reperfusion injury has been supported by the observation that interventions started before reperfusion can reduce infarct size, as discussed below $[4,5]$. Studies in animals suggest that lethal reperfusion injury accounts to up to $50 \%$ of the final size of a myocardial infarction.

Clinically, reperfusion injury may be seen in four different types of cardiac dysfunction: myocardial stunning - that is persistent mechanical dysfunction despite restored blood flow which is usually reversible within weeks; the noreflow phenomenon after opening of an infarcted coronary artery; reperfusion arrhythmia; and lethal, irreversible injury of the myocardium. In the recent years, rapid revascularization was instituted to prevent reperfusion injury. From a clinical view, currently there seems to be no potential to further reduce infarct size by faster restoration of blood flow. Therefore other than mechanical strategies to reduce reperfusion injury and in consequence infarct size are highly welcome to improve the outcome of the patients. To further understand potential strategies, the molecular mechanisms contributing to reperfusion injury are of importance.

Signals contributing to reperfusion injury: The signals contributing to reperfusion injury have been recently reviewed in detail [5]. Major contributors are as follows.

Oxidative stress [6]: which is discussed as the oxygen paradox since the reoxygenation of ischemia generates a myocardial injury that exceeds the injury of ischemia alone. Oxidative stress diminishes the cardioprotective effects of nitric oxide, leading to more neutrophil activation, increased levels of superoxide radicals, and diminished myocardial blood flow.

Increased intracellular calcium: occurring secondary to the ischemic damage of the sarcolemmal membranes and to the oxidative stress-induced dysfunction of the sarcoplasmic reticulum [7]. The calcium excess induces cardiac myocytes death by hypercontracture and opening of the mitochondrial permeability transition pore (mPTP), a molecule recently identified as one of the most important targets in reperfusion injury [8].

The rapid restoration of physiologic $\mathrm{pH}$ during reperfusion: which follows the washout of lactic acid. This leads to the activation of the sodium-hydrogen exchanger, finally leading to MPTP opening [9].

Inflammation: leading to neutrophil accumulation and their transmigration into the myocardial tissue. These neutrophils cause vascular plugging and release degradative enzymes and reactive oxygen species [10].

In addition to these more traditional factors, the reperfusion injury salvage kinase (RISK) pathway and its effector, the MPTP, have been currently postulated to be centrally involved in reperfusion injury $[8,11,12]$. Opening of the mPTP, which releases calcium from the mitochondria and leads to intracellular calcium overload, does not occur in ischemia but is a key determinant of the first few minutes of myocardial reperfusion. Some authors discuss that this pathway could serve as the final effector of the above discussed contributors to reperfusion injury.

Treatment in humans: Whereas in animal studies many agents were successful to reduce reperfusion injury, the translation of these results into the clinical setting has been disappointing for many years [5]. Several groups tried to target oxidative stress with antioxidants or nitric oxide supplementation. These trials had negative results $[5,13,14]$. Comparably, trials of inhibition of the sodium-hydrogen exchanger, which have been successful in animals by reducing the intracellular calcium overload in reperfusion and by delaying $\mathrm{pH}$ normalization in the reperfused myocardium, failed in humans [15], as have various measures to reduce the inflammatory damage induced by neutrophils [5]. Trials with metabolic modulation such as glucose-insulin-potassium infusions or magnesium therapy had inconclusive results. Together, these measures addressing the traditionally seen contributors to reperfusion injury were, at least in humans, not successful to clearly reduce myocardial damage.

Some attempts have clinically been done to treat the no-reflow phenomenon. The administration of platelet glycoprotein GP Ilb/lla blockers or thrombus aspiration improves reflow after vessel reopening, with limited influence of infarct size. Adenosine is widely used to treat slow-flow phenomena in the infarct-related artery. Of note, the administration of adenosine as an anti-inflammatory and vasodilatatory agent during reperfusion could reduce infarct size in humans, making adenosine currently the only clinically used drug which improves both reflow and infarct size. Adenosine, however, did not reduce clinical end points, which was the primary end point of this study [16].

Three strategies are currently discussed as innovative treatment modalities for reperfusion injury in acute myocardial infarction: mechanical postconditioning, pharmacological postconditioning with 
substances influencing the RISK pathway and the MPTP, and hypothermia.

Mechanical postconditioning: In 2003, Zhao and coworkers could demonstrate that short periods of ischemia and reperfusion applied immediately after reopening of an infarct-related artery could reduce reperfusion injury and infarct size, a concept referred to as ischemic postconditioning [17]. In animal studies, ischemic postconditioning involves all major contributors to reperfusion injury including the RISK pathway and the mPTP. Various smaller clinical studies showed in humans that repetitive inflations of an angioplasty balloon after reopening of the infarct-related artery reduces infarct size, wall-motion scores and comparable end points [18-20]. A related strategy, which has also shown to be effective in animals and humans, is the remote ischemic postconditioning. Here, transient episodes of ischemia and reperfusion in a remote organ - that is, skeletal muscle - protect the heart from reperfusion injury. A very elegant randomized trial could show that the simple, non-invasive, repetitive inflation of a standard blood pressure cuff in patients with acute myocardial infarction reduces infarct size when applied before the reopening of the infarct-related artery [21]. Given the confirmation of this finding in larger trials, mechanical, remote postconditioning could be an easy and safe measure to reduce reperfusion injury.

Pharmacologic postconditioning: Extensive preclinical evidence showed that pharmacologic activation of the RISK pathway or prevention of MPTP opening reduces reperfusion injury. Some drugs addressing the RISK pathway have been tested in proof-of-concept studies in patients. Of those, inhibition of the protein kinase C-isoform delta with the substance KAl-9803 given into the infarct-related area immediately before reperfusion [22] and administration of high-dose atorvastatin before $\mathrm{PCl}$ [23] are the most promising, both showing favorable effects in about 150 randomized patients. Another clinical trial addressed directly the mPTP. In a small pilot trial including 58 patients, the i.v. administration of cyclosporine $(2.5 \mathrm{mg} / \mathrm{kg})$, known to inhibit mPTP opening, reduced infarct size and the release of cardiac markers [24]. Due to the limited numbers of patients, however, it is not clear whether those interventions result in the reduction of clinical endpoints.

Hypothermia: For a couple of years, it has been known that in animals hypothermia instituted before the reopening of an infarct-related coronary artery reduces reperfusion injury and thus infarct size [25]. For infarct size reduction, hypothermia has to be instituted before reperfusion [26], whereas cooling directly after vessel reopening is unsuccessful in most cases [26]. It is discussed that hypothermia prevents the reactive hyperemia in early reperfusion.

Few studies examined the effects of hypothermia in patients with acute myocardial infarction. Dixon and colleagues could show that therapeutic hypothermia is safe in myocardial infarction patients, however, without influencing outcomes [27]. Wolfrum and colleagues showed in patients with myocardial infarction subjected to CPR that institution of therapeutic hypothermia before revascularization did not prolong door-to-balloon times [28]. Two yet unpublished studies, the COOL-MI study and the ICE-IT study, showed no effect on infarct size in the total population. However, in patients with anterior myocardial infarction cooled to $<35^{\circ} \mathrm{C}$ at the time of reperfusion, infarct sizes were roughly halved [29]. The treatment of awake patients not subject to CPR is, however, limited due to counter-regulatory processes such as shivering, which leads to increased oxygen demand and workload of the heart. Future studies will test hypothermia in patients with myocardial infarction, using a straightforward adjunctive treatment with buspirone and meperidine to prevent shivering. In this setting, clear treatment protocols are necessary to avoid prolongation of the door-toballoon time in patients with myocardial infarction.

Problems still to face: Signaling: Some conditions have been identified to inhibit salvage from reperfusion damage like myocardial hypertrophy, diabetes, age, and hypercholesterolemia (reviewed in [30]). All these conditions are associated with limited ability to successfully activate the RISK pathway. Either they increase the threshold of mechanical postconditioning or they abolish myocardial salvage at all. As these conditions are highly present in the patient population, further research may be of relevance to reestablish susceptibility to modified reperfusion strategies.

Timing: All proposed strategies to prevent reperfusion damage assume a relevant amount of reperfusable myocardium that is waiting to be salvaged. All planned measures to reduce reperfusion damage before revascularization should preferably be applied in a very short time.
Infarct size: Hard clinical endpoints can only be met when there is a significant clinical benefit to be achieved. Posterior infarcts or smaller infarcts of the anterior region only affecting small or medium-sized vessels do not go along with high mortality and therefore there are not many lives to be saved by an optimal reperfusion strategy.

Conclusion: Reperfusion injury contributes to up to $50 \%$ of the total myocardial damage. In spite of many successful results in animals, the translation into the clinical setting has been disappointing for many years. Recently, mechanical, remote ischemic postconditioning as well as pharmacologic postconditioning with substances addressing the RISK pathway and the MPTP have shown positive effects on surrogate end points in patients. Hypothermia may be another option in the treatment of reperfusion injury. Larger studies exploring the potential of these therapeutic options to influence clinically relevant endpoints are eagerly awaited. Currently, the evidence is insufficient to permit a widespread clinical use of those interventions.

\section{References}

1. Sobel BE, Breshnan GF, Shell WE, Yoder RD: Estimation of infarct size in man and its relation to prognosis. Circulation 1972, 46:640-648.

2. Reimer KA, Lowe JE, Rasmussen MM, Jennings RB: The wavefront phenomenon of ischemic cell death. 1. Myocardial infarct size vs duration of coronary occlusion in dogs. Circulation 1977, 56:786-794.

3. Jennings RB, Sommers HM, Smyth GA, Flack HA, Linn H: Myocardial necrosis induced by temporary occlusion of a coronary artery in the dog. Arch Pathol 1960, 70:68-78.

4. Ovize M, Baxter GF, Di Lisa F, Ferdinandy P, Garcia-Dorado D, Hausenloy DJ, et al: Postconditioning and protection from reperfusion injury: where do we stand? Cardiovasc Res 2010, 87:406-423.

5. Yellon DM, Hausenloy DJ: Myocardial reperfusion injury. N Engl J Med 2007, 357:1121-1135.

6. Zweier JL: Measurement of superoxide-derived free radicals in the reperfused heart. Evidence for a free radical mechanism of reperfusion injury. J Biol Chem 1988, 263:1353-1357.

7. Piper HM, Garcia-Dorado D, Ovize M: A fresh look at reperfusion injury. Cardiovasc Res 1998, 38:291-300.

8. Hausenloy DJ, Yellon DM: The mitochondrial permeability transition pore: its fundamental role in mediating cell death during ischaemia and reperfusion. J Mol Cell Cardiol 2003, 35:339-341.

9. Kim JS, Jin Y, Lemasters JJ: Reactive oxygen species, but not $\mathrm{Ca}^{2+}$ overloading, trigger $\mathrm{pH}$ - and mitochondrial permeability transitiondependent death of adult rat myocytes after ischemia-reperfusion. Am J Physiol Heart Circ Physiol 2006, 290:H2024-H2034.

10. Vinten-Johansen J: Involvement of neutrophils in the pathogenesis of lethal myocardial reperfusion injury. Cardiovasc Res 2004, 61:481-497.

11. Hausenloy DJ, Tsang A, Mocanu MM, Yellon DM: Ischemic preconditioning protects by activating pro-survival kinases at reperfusion. Am J Physiol Heart Circ Physiol 2005, 288:H971-H976.

12. Hausenloy DJ, Yellon DM: New directions for protecting the heart against ischaemia/reperfusion injury: targeting the Reperfusion Injury Salvage Kinase (RISK)-pathway. Cardiovasc Res 2004, 61:448-460.

13. Effect of 48-h intravenous trimetazidine on short- and long-term outcomes of patients with acute myocardial infarction, with and without thrombolytic therapy. A double-blind, placebo-controlled, randomized trial. Eur Heart J 2000, 21:1537-1546.

14. Flaherty JT, Pitt B, Gruber JW, Heuser RR, Rothbaum DA, Burwell LR, et al: Recombinant human superoxide dismutase (h-SOD) fails to improve recovery of ventricular function in patients undergoing coronary angioplasty for acute myocardial infarction. Circulation 1994, 89:1982-1991

15. Zeymer U, Suryapranata H, Monassier JP, Opolski G, Davies J, Rasmanis G, et al: The $\mathrm{Na}^{+} / \mathrm{H}^{+}$exchange inhibitor eniporide as an adjunct to early reperfusion therapy for acute myocardial infarction: Results of the evaluation of the safety and cardioprotective effects of eniporide in acute myocardial infarction (ESCAMI) trial. JACC 2001, 38:1644-1650.

16. Ross AM, Gibbons RJ, Stone GW, Kloner RA, Alexander RW: A randomized, double-blinded, placebo-controlled multicenter trial of adenosine as an adjunct to reperfusion in the treatment of acute myocardial infarction (AMISTAD-II). JACC 2005, 45:1775-1780.

17. Zhao ZQ, Corvera JS, Halkos ME, Kerendi F, Wang NP, Guyton RA, et al: Inhibition of myocardial injury by ischemic postconditioning during reperfusion: comparison with ischemic preconditioning. Am J Physiol Heart Circ Physiol 2003, 285:H579-H588. 
18. Laskey WK: Brief repetitive balloon occlusions enhance reperfusion during percutaneous coronary intervention for acute myocardial infarction: a pilot study. Catheter Cardiovasc Interv 2005, 65:361-367.

19. Ma X, Zhang X, Li C, Luo M: Effect of postconditioning on coronary blood flow velocity and endothelial function and LV recovery after myocardial infarction. J Intervent Cardiol 2006, 19:367-375.

20. Staat P, Rioufol G, Piot C, Cottin Y, Cung TT, L Huillier I, et al: Postconditioning the human heart. Circulation 2005, 112:2143-2148.

21. Botker HE, Kharbanda R, Schmidt MR, Bottcher M, Kaltoft AK, Terkelsen CJ, et al: Remote ischaemic conditioning before hospital admission, as a complement to angioplasty, and effect on myocardial salvage in patients with acute myocardial infarction: a randomised trial. Lancet 2010, 375:727-734.

22. DELTA Investigators: Intracoronary KAl-9803 as an adjunct to primary percutaneous coronary intervention for acute ST-segment elevation myocardial infarction. Circulation 2008, 117:886-896.

23. Patti G, Pasceri V, Colonna G, Miglionico M, Fischetti D, Sardella G, et al: Atorvastatin pretreatment improves outcomes in patients with acute coronary syndromes undergoing early percutaneous coronary intervention: results of the ARMYDA-ACS randomized trial. JACC 2007, 49:1272-1278

24. Piot C, Croisille $P$, Staat $P$, Thibault $H$, Rioufol $G$, Mewton $N$, et al: Effect of cyclosporine on reperfusion injury in acute myocardial infarction. $N$ Engl J Med 2008, 359:473-481

25. Miki T, Liu G-S, Cohen MV, Downey JM: Mild hypothermia reduces infarct size in the beating rabbit heart: a practical intervention for acute myocardial infarction? Basic Res Cardiol 1998, 93:372-383.

26. Götberg M, Olivecrona G, Engblom H, Ugander M, van der Pals J, Heiberg E, et al: Rapid short-duration hypothermia with cold saline and endovascular cooling before reperfusion reduces microvascular obstruction and myocardial infarct size. BMC Cardiovasc Disord 2008, 8:7

27. Dixon SR, Nguyen TT, O Neill WW, Whitbourn RJ, Dae MW, Grube E, et al: Induction of mild systemic hypothermia with endovascular cooling during primary percutaneous coronary intervention for acute myocardial infarction. JACC 2002, 40:1928-1934.

28. Wolfrum S, Pierau C, Radke PW, Schunkert H, Kurowski V: Mild therapeutic hypothermia in patients after out-of-hospital cardiac arrest due to acute ST-segment elevation myocardial infarction undergoing immediate percutaneous coronary intervention. Crit Care Med 2008, 36:1780-1786.

29. Tissier R, Chenoune M, Ghaleh B, Cohen MV, Downey JM, Berdeaux A: The small chill: mild hypothermia for cardioprotection? Cardiovasc Res 2010, 88:406-414.

30. Przyklenk K: Efficacy of cardioprotective postconditioning strategies in aging and diabetic cohorts: the co-morbidity conundrum. Drugs Aging 2011, 28:331-341.

\section{COOLING: CROSSING BORDERS}

\section{A23}

Intracranial pressure and hypothermia

Claudius Thomé

Department of Neurosurgery, Innsbruck Medical University, Innsbruck, Austria Critical Care 2012, 16(Suppl 2):A23

Although the neuroprotective potential of hypothermia is well known and has been established experimentally, its clinical use is limited to selected indications [1], as large trials have yielded disappointing results [2]. This has been mainly attributed to the side effects of hypothermia in critically ill patients and problems with rewarming.

Intracranial hypertension is a major problem in neurocritical care and particularly in patients with subarachnoid hemorrhage $(\mathrm{SAH})$ and traumatic brain injury (TBI), causing death if uncontrolled. As trials on prophylactic hypothermia, for example, for TBI have not been successful in improving outcome, its routine use can currently not be recommended. However, there are many literature reports demonstrating enormous efficacy of hypothermia to reduce elevated intracranial pressure (ICP). Mechanisms of action are thought to be the reduction of metabolism and perfusion and the reduction of edema besides others. Studies have indicated that therapeutic efficacy is sufficient for ICP control at mild hypothermia of $35^{\circ} \mathrm{C}$, thus minimizing detrimental effects. In desperate clinical situations hypothermia is used to control intracranial hypertension both for TBI and $\mathrm{SAH}$, but it has recently been applied only as a last resort. Other second-tier therapies and surgical maneuvers like decompressive craniectomy have been popularized instead, although their efficacy is still questioned as well. The knowledge and experience with therapeutic hypothermia has advanced in recent years and the problems of side effects and most importantly rewarming can be better addressed [3]. The latter has been a tremendous problem in patients with uncontrollable ICP, as despite its initial efficacy ICP problems recurred, if hypothermia was stopped prematurely. This goes in line with a recent metaanalysis that stressed the importance of prolonged hypothermia (48 hours to 5 days) and of slow rewarming $\left(<1^{\circ} \mathrm{C} / 4\right.$ hours). As a consequence the Eurotherm3235Trial was initiated to investigate the effect of hypothermia particularly for intracranial pressure reduction [4]. It has to be awaited whether this will foster the use of hypothermia to treat elevated ICP or whether we will stick with the policy of controlled normothermia.

References

1. Rivera-Lara L, Zhang J, Muehlschlegel S: Therapeutic hypothermia for acute neurological injuries. Neurotherapeutics 2012, 9:73-86.

2. Clifton GL, Miller ER, Choi SC, Levin HS, McCauley S, Smith KR Jr, Muizelaar JP, Wagner FC Jr, Marion DW, Luerssen TG, Chesnut RM, Schwartz M: Lack of effect of induction of hypothermia after acute brain injury. N Engl J Med 2001, 344:556-563.

3. Polderman $\mathrm{KH}$, Herold I: Therapeutic hypothermia and controlled normothermia in the intensive care unit: practical considerations, side effects, and cooling methods. Crit Care Med 2009, 37:1101-1120.

4. Andrews PJ, Sinclair HL, Battison CG, et al: European society of intensive care medicine study of therapeutic hypothermia $\left(32^{\circ}-35^{\circ} \mathrm{C}\right)$ for intracranial pressure reduction after traumatic brain injury (the Eurotherm3235 Trial). Trials 2011, 12:12-18.

\section{A24}

Rewarming: facts and myths from the neurological perspectives

Erich Schmutzhard*, Marlene Fischer, Anelia Dietmann, Raimund Helbok, Gregor Broessner

Department of Neurology, Neurocritical Care Unit, Medical University

Hospital Innsbruck, Austria

Critical Care 2012, 16(Suppl 2):A24

Standard operating procedures both for scientific study protocols as well as in routine daily practice stress the importance of slow rewarming after targeted temperature management/moderate therapeutic hypothermia (32 to $34^{\circ} \mathrm{C}$ ) [1-3]. The recommended rewarming speed ranges from 0.1 to $0.4^{\circ} \mathrm{C}$ /hour, the former allowing a minimum of 24 hours to reach normothermia levels whereas in case of the latter the normothermic temperature levels are reached within 6 to 8 hours. It has been well known and it is widely accepted that abrupt temperature changes, with insufficient temperature control methods, cause change in energy expenditure and intracranial pressure and a negative effect onto the cerebral perfusion pressure and cerebral blood flow. In avalanche survivors the so-called afterdrop has been described to be an additional potentially dangerous condition [4,5]; hypercapnia has been shown to increase the core temperature cooling rate in snow burial victims; furthermore, shivering influences both cooling and rewarming and the cooling afterdrop [4,5].

In the rat model the speed of rewarming plays a crucial role in the development of acute lung injury in intestinal ischemia treated with therapeutic hypothermia. Reactive oxygen species have been shown to play an important role in the pathogenesis of various injuries, including brain injury and lung injury [6]. Besides this, inflammatory reaction and nitric oxide levels are clearly influenced both by therapeutic hypothermia and speed of rewarming. It might be surmised that more gradual rewarming and the addition of anti-inflammatory drugs during this period of rewarming $[7,8]$ might add to or even enhance the neuroprotective effect of therapeutic hypothermia/targeted temperature management $[9,10]$. This assumption is underlined by the findings that biogenic amines that have been demonstrated to protect cells from apoptotic cell death (for example, serotonin and dopamine) protect cells against rewarminginduced active oxygen species formation and apoptosis [11]. Thus, it is correctly hypothesized that adding drugs containing these biogenic amines or releasing endogenously serotonin and dopamine might help to prevent potential negative side effects of rewarming [11]. 
Most of the knowledge of post-hypothermic rewarming effects has been gained in experimental settings $[1,12,13]$. More than 10 years ago the neuroprotective effect visualized by amyloid precursor protein positive axonal swellings, quantifying them per unit area and, thus, serving as marker of both pathophysiologic and neuroprotective effect respectively, has been shown to be reversed completely when normothermia was achieved very rapidly within 20 minutes $[3,14]$. The originally documented axonal protection was not only eliminated by this rapid rewarming but, in fact, the overall burden of axonal damage was dramatically increased, reaching a virtual doubling of the numbers of damaged axons seen per unit area [3]. Not only amyloid precursor protein accumulation but also neurofilament compaction was seen to be exacerbated by rapid rewarming [3].

Besides this and other neuropathological effects there is now clear evidence that cerebral microcirculation, both in traumatic brain injury and in other severe intracranial diseases, is structurally and functionally perturbed and that this perturbation can be attenuated by hypothermia followed by slow rewarming $[2,15]$. Similar to the situation with axonal injury, discussed above, the hypothermic protection against cerebral microcirculation impairment can be reversed or the injury even exacerbated by rapid post-hypothermic rewarming [2]. In addition, compelling evidence has been found that traumatically induced or hypoxia-induced generation of oxygen radicals significantly contributes to secondary insult onto brain cells but also damaging endothelium and smooth muscle cells [13]. When employing hypothermic intervention followed by slow rewarming, significant vascular protection was provided; in particular, the generation of oxygen radicals was reduced [14]. The protective effect of hypothermia, once again, is not only a function of time of initiating target temperature and overall duration of hypothermia. Even more importantly, all these positive effects are reversed by rapid rewarming enhancing vascular dysfunction following neuronal injury [10]. Povlishock and Wei clearly state that in the context of traumatically induced axonal damage, microvascular dysfunction, and cerebral contusion, any potentially beneficial effect of hypothermic intervention is consistently reversed and the lesion even exacerbated when posttraumatic hypothermia is followed by rapid rewarming [2]. Most likely, both direct and indirect mitochondrial perturbation together with processes mediated by free radicals influence the ensuing biology and pathophysiology. Post-hypothermic rewarming rates and the potential adverse consequences of rapid rewarming are closely observed in the field of transplantation medicine wherein hypothermic organ maintenance and rewarming are integral to successful organ viability and subsequent transplantation. Rapid rewarming is highly damaging in case of liver transplantation, most likely due to rapid ATP depletion, energy failure and oxygen radical production associated with hepatic mitochondrial damage. Extrapolation from this body of experimental and human medical knowledge clearly indicates/allows the statement that any type of targeted temperature management/therapeutic hypothermia needs to be followed both by very slow rewarming on the one hand and maintenance of normothermia after reaching the normothermia level on the other hand [16-19]. Exactly this aspect of slow/very slow rewarming has been partially neglected in most recently published studies [19,20]; in particular, on therapeutic hypothermia in traumatic brain injury [21]. In view of the ongoing pathophysiologic processes leading to secondary neuronal damage over a period of more than 5 days post trauma, not only that the duration of therapeutic hypothermia but also the speed of rewarming $\left(0.4^{\circ} \mathrm{C} /\right.$ hour $)$ need to be questioned and might be interpreted as the major cause of the negative study outcome.

Every researcher, when compiling a study protocol, as well as every clinician is strongly advised to accept the impact of duration of hypothermia and speed/rate of rewarming as being crucial elements both in research and clinical practice. Although it might correctly be surmised that the duration of therapeutic hypothermia is associated with the incidence and intensity of complications, the same might hold true for too short a duration and too quick a rewarming rate. Further studies are needed to evaluate various durations ( 1 day vs. 3 or 5 days, vs. even longer) both in traumatic brain injury but also in other neurologic diseases that trigger off secondary pathophysiological processes such as ischemic stroke (concept of penumbra), spontaneous intracerebral hemorrhage (peri-hematoma edema), traumatic brain injury or spontaneous aneurysmatic subarachnoid hemorrhage with pathophysiologic processes going on for days and even weeks after the acute ictus. In view of these considerations, prolonging therapeutic hypothermia and, in particular, slowing the rate of rewarming might be the crucial clue to achieve the best possible benefit from targeted temperature management/moderate therapeutic hypothermia without counteracting these benefits by too speedy a rewarming rate. In addition, maintaining normothermia after hypothermia and rewarming seems to be essential in order to avoid the negative rebound effects $[22,23]$.

Finally, it needs again to be noted that a too rapid rewarming might lead to vasodilatation, thus aggravating intracranial pressure, reducing cerebral perfusion pressure and leading to a negative effect on overall neurological outcome $[1,19]$.

\section{References}

1. Alzaga AG, Cerdan M, Varon J: Therapeutic hypothermia. Resuscitation 2006, 70:369-380.

2. Povlishock JT, Wei EP: Posthypothermic rewarming considerations following traumatic brain injury. J Neurotrauma 2009, 26:333-340.

3. Suehiro E, Ueda Y, Wei EP, Kontos HA, Povlishock JT: Posttraumatic hypothermia followed by slow rewarming protects the cerebral microcirculation. J Neurotrauma 2003, 20:381-390.

4. Grissom CH, Harmston CH, McAlpine JC, et al: Spontaneous endogenous core temperature rewarming after cooling due to snow burial. Wilderness Environ Med 2010, 21:229-235.

5. Muller MD: 'Rewarming' an important issue from the cold: simulated avalanche survival and the physiology of afterdrop. Wilderness Environ Med 2011, 22:98-99.

6. Kim K, Jo YH, Rhee JE, et al: Effect of speed of rewarming and administration of anti-inflammatory or anti-oxidant agents on acute lung injury in an intestinal ischemia model treated with therapeutic hypothermia. Resuscitation 2010, 81:100-105.

7. Bisschops LL, Hoedemaekers CM, Mollnes TE, van der Hoeven JG: Rewarming after hypothermia after cardiac arrest shifts the inflammatory balance. Crit Care Med 2012, 40:1136-1142.

8. Kamps M, Bisschops LA, van der Hoeven JG, Hoedemaekers CW: Hypothermia does not increase the risk of infection: a case control study. Crit Care 2011, 15:R48.

9. Broessner $G$, Lackner $P$, Fischer $M$, et al: Influence of prophylactic, endovascularly based normothermia on inflammation in patients with severe cerebrovascular disease: a prospective, randomized trial. Stroke 2010, 41:2969-2972.

10. Suehiro E, Povlishock JT: Exacerbation of traumatically induced axonal injury by rapid posthypothermic rewarming and attenuation of axonal change by cyclosporine A. J Neurosurg 2001, 94:493-498.

11. Talaei F, Bouma HR, Van der Graaf AC, et al: Serotonin and dopamine protect from hypothermia/rewarming damage through the $\mathrm{CBS} / \mathrm{H} 2 \mathrm{~S}$ pathway. PLoS One 2011, 6:e22568.

12. Ma M, Matthews BT, Lampe JW, et al: Immediate short-duration hypothermia provides long-term protection in an in vivo model of traumatic axonal injury. Exp Neurol 2009, 215:119-127.

13. Wei EP, Hamm RJ, Baranova Al, Povlishock JT: The long-term microvascular and behavioural consequences of experimental traumatic brain injury after hypothermic intervention. I Neurotrauma 2009, 26:527-537.

14. Ueda Y, Suehiro E, Wei EP, Kontos HA, Povlishock JT: Uncomplicated rapid posthypothermic rewarming alters cerebrovascular responsiveness. Stroke 2004, 35:601-601.

15. Bisschops LL, Hoedemaekers CW, Simons KS, van der Hoeven JG: Preserved metabolic coupling and cerebrovascular reactivity during mild hypothermia after cardiac arrest. Crit Care Med 2010, 38:1542-1547.

16. Bisschops LL, van Alfen N, Bons S, van der Hoeven JG, Hoedemaekers CW: Predictors of poor neurologic outcome in patients after cardiac arrest treated with hypothermia: a retrospective study. Resuscitation 2011, 82:696-701.

17. Burnsed J, Quigg M, Zanelli S, Goodkin HP: Clinical severity, rather than body temperature, during the rewarming phase of therapeutic hypothermia affect quantitative EEG in neonates with hypoxic ischemic encephalopathy. J Clin Neurophysiol 2011, 28:10-14.

18. Davies AR: Hypothermia improves outcome from traumatic brain injury. Crit Care Resusc 2005, 7:238-243.

19. Jiang JY, Yang XF: Current status of cerebral protection with mild-tomoderate hypothermia after traumatic brain injury. Curr Opin Crit Care 2007, 13:153-155.

20. Clifton GL, Miller ER, Choi SC, et al: Lack of effect of induction of hypothermia after acute brain injury. N Engl J Med 2001, 344:556-563. 
21. Clifton GL, Valadka A, Zygun D, et al: Very early hypothermia induction in patients with severe brain injury (the National Acute Brain Injury Study: Hypothermia II): a randomized trial. Lancet Neurol 2011, 10:131-139.

22. Broessner $G$, Beer $R$, Lackner $P$, et al: Prophylactic, endovascularly based, long-term normothermia in ICU patients with severe cerebrovascular disease: bicenter prospective, randomized trial. Stroke 2009, 40:e657-e665.

23. Fischer $M$, Lackner $P$, Beer $R$, et al: Keep the brain cool - endovascular cooling in patients with severe traumatic brain injury: a case series study. Neurosurgery 2011, 68:867-873.

\section{A25}

Rewarming: facts and myths from the systemic perspective

$\checkmark$ Scaravilli $^{1 *}$, D Bonacina ${ }^{1}$, G Citerio $^{2}$

${ }^{1}$ Department of Experimental Medicine, University of Milano-Bicocca, Ospedale San Gerardo, Monza, Italy; ${ }^{2}$ Neuroanesthesia and Neurointensive Care Unit, Department of Perioperative Medicine and Intensive Care, Ospedale San Gerardo, Monza, Italy

Critical Care 2012, 16(Suppl 2):A25

Introduction: Rewarming is a delicate phase of therapeutic hypothermia $\mathrm{TH}$. Adverse consequences of rewarming on the whole body may seriously limit the protective effects of hypothermia, leading to secondary injury. Thus, understanding, predicting, and managing possible systemic side effects of rewarming is important for guaranteeing TH efficacy. The aim of this brief report is to describe rewarming effects from a systemic perspective.

Hemodynamics and imbalance in oxygen consumption and delivery: $\mathrm{TH}$ linearly decreases the metabolic rate of homeothermic organisms. During the cooling process, tissue oxygen consumption $\left(\mathrm{VO}_{2}\right)$ slows by roughly $6 \% /{ }^{\circ} \mathrm{C}$ reduction in body temperature $[1,2]$, obeying the van't Hoff-Arrhenius law, which states that the rate of a biochemical reaction is halved for each $10^{\circ} \mathrm{C}$ decrease in temperature. The reduction in brain metabolism is similar [3].

In contrast, during rewarming, the possible appearance of a mismatch between total body oxygen demand and oxygen delivery $\left(\mathrm{DO}_{2}\right)[4]$ has been recognized since the pioneer works of Hegnauer and colleagues on dogs [5] and Bigelow on humans [6]. Bigelow has described this side effect of rewarming as rewarming shock: 'This syndrome of acute acidosis, or rewarming shock, was characterized by a progressive decline in blood $\mathrm{pH}$ [...] associated with respiratory inadequacy [...]. A fall in blood pressure and tachycardia were features in some cases'. In more recent studies, rewarming shock after moderate TH seems to be a more infrequent eventuality, probably because TH management has been completely changed by the advent of ICUs and a far less hypothermic regimen. The mismatch between oxygen supply and consumption during rewarming could depend on numerous factors, including metabolic rate, abnormalities in oxygen extraction, cardiac output (CO), circulating blood volume, regional blood flow, $\mathrm{pH}$, blood viscosity, and a shift in the hemoglobin dissociation curve. The physiopathology of this side effect of rewarming is not known. Rewarming from hypothermia is such a complex and metabolism-pervasive process to alter all of the possible determinants of a $\mathrm{VO}_{2} / \mathrm{DO}_{2}$ mismatch. Of the possible determinants of $\mathrm{VO}_{2} / \mathrm{DO}_{2}$, cardiac dysfunction has been the most investigated. Cooling determines a proportional decrease in cardiac output [1], heart rate, and mean arterial blood pressure, with no change in stroke volume and increased peripheral vascular resistance. During the maintenance stage of $\mathrm{TH}$, the decrease in metabolic rate is equal to or greater than the decrease in cardiac output, and alteration of oxygen delivery is not a matter of concern. Preliminary clinical studies [7] and a recent meta-analysis [8] have shown a decrease in myocardial ischemic injury. Many of the alterations in the cardiovascular system occurring during hypothermia completely reverse during rewarming. Therefore, the rewarming phase could lead to a permanent deterioration of myocardial function and cardiac output. The pathophysiological mechanism underlying cardiac dysfunction induced by hypothermia rewarming has been studied by Tveita's group, first in vitro using a rat left ventricular papillary muscle [9] and then in vivo [10] in an intact rat model. These studies showed how postrewarming systolic left ventricular dysfunction can be related to decreased myofibrillar $\mathrm{Ca}^{2+}$ sensitivity due to increased troponin $\mathrm{C}$ phosphorylation. In addition, Blair and colleagues [11] and Morray and Pavlin [12] documented an increase in total oxygen consumption to values above prehypothermic controls in a dog model of rewarming after deep hypothermia. The authors suggested many possible explanations for this event. First, heterogeneous blood flow distribution [13] during hypothermia may determine areas of oxygen debt, with decreased or absent perfusion, that become hypoxic and generate lactate. During rewarming, these areas are reperfused and lactate re-enters normal oxidative pathways, consuming oxygen in the process. Second, with a return to normothermia, free radical oxidation $[14,15]$ and inflammatory response to injury $[16,17]$ could resume, leading to nonrespiratory utilization of oxygen and an increase of $\mathrm{VO}_{2}$ over pre-injury control. Third, shivering can occur during rewarming as a response to deviations from the temperature set point. The shivering response to maintain a constant core temperature is a concerted reaction involving skeletal muscle contraction and peripheral vasoconstriction. When shivering occurs during rewarming, it is associated with increased $\mathrm{VO}_{2}[18,19]$ and hemodynamic instability [20].

Cain and Bradley 21] and Schumacker and colleagues [22] have described abnormalities of peripheral oxygen extraction in dogs during hypothermia, even with adequate oxygen delivery. An alteration in the temperature transition of oxidative phosphorylation has been documented in an animal model. Leducq and colleagues presented evidence for an abnormal pattern of oxidative phosphorylation control that correlated with a transition in mitochondrial permeability and persisted after rewarming [23]. This phenomenon may cause alterations in oxygen utilization during and after rewarming.

Kondratiev and colleagues addressed the problem of oxygen supply in a rat model of deep hypothermia $\left(15^{\circ} \mathrm{C}\right)$ and rewarming [24]. The experiment demonstrated a reduction in cardiac output and oxygen delivery after prolonged deep hypothermia $\left(15^{\circ} \mathrm{C}\right.$ for 5 hours) compared with less prolonged exposure. The rewarming-related rightward shift of the oxygen hemoglobin saturation curve, which facilitates oxygen dissociation at the tissue level, compensated for compromised peripheral oxygen transport, leading to a stable oxygen supply. Knowing the events causing $\mathrm{VO}_{2} / \mathrm{DO}_{2}$ mismatch during rewarming is important in this phase of $\mathrm{TH}$ for monitoring and assuring adequate cerebral and whole body oxygen delivery. Low oxygen delivery accounts for the development of secondary injury, which limits the safety and effectiveness of $\mathrm{TH}$. With this perspective in mind, we can suggest various measures to limit $\mathrm{VO}_{2} / \mathrm{DO}_{2}$ mismatch during rewarming.

First, rewarming after TH should be done slowly and in a controlled manner [25]. Eshel, in a rat model of $\mathrm{TH}$, showed how rapid rewarming from moderate hypothermia is associated with more acute hemodynamic alterations compared with slow rewarming [25]. Similar effects were described in humans [26] and pediatric patients [27] undergoing TH for hypoxic ischemic encephalopathy and deep intraoperative hypothermia $\left(27^{\circ} \mathrm{C}\right)$, respectively, as well as in the work of Hanhela and colleagues [28] on adults undergoing cardiopulmonary bypass for cardiac surgery.

Second, controlling pain, sedation, and preventing shivering should limit oxygen consumption. Michenfelder and colleagues [29], Rodriguez and colleagues [30], and Zwischenberger and colleagues [31] demonstrated that the suppression of shivering by neuromuscular blockade is an effective method for diminishing $\mathrm{VO}_{2}$. More recently, Badjata and colleagues [32] proposed a simple shivering grading tool, the Bedside Shivering Assessment Scale (BSAS), developed by assessing the correlation of bedside shivering and systemic metabolic stress quantified by indirect calorimetry. Using clinical observation of muscle involvement, the BSAS provides an accurate representation of shivering-related oxygen consumption. Accurately defining shivering intensity assures the possibility of a stepwise treatment for shivering. We recommend initially managing shivering with nonsedating interventions, such as correcting hypomagnesemia, or a serotonin (5- $\mathrm{TH}) 1 \mathrm{~A}$ partial agonist like buspirone or meperidine. Meperidine has been demonstrated to effectively reduce $\mathrm{VO}_{2}$ augmentation associated with postoperative shivering at a dosage that does not cause respiratory depression [33]. When these first line interventions fail, sedation with shortacting sedative agents and neuromuscular blockade can be used.

Third, oxygen content and transport should be optimized. Anemia and arterial desaturation must be avoided during rewarming. To date, no clinical trials have examined hemodynamic optimization in patients that have undergone $\mathrm{TH}$, least of all during rewarming, and no evidence is currently available to indicate the best strategy for hemodynamic support in such a critical phase. We suggest a strict control of hemodynamics, with the aim of guaranteeing adequate oxygen delivery and avoiding $\mathrm{VO}_{2} / \mathrm{DO}_{2}$ mismatch, using at least continuous arterial pressure monitoring, volume balance and urine output surveillance, and frequent serum lactate 
measurements. In the case of hemodynamic instability, advanced monitoring capable of finer management could be useful. Thus, in this context, echocardiography and goal-directed hemodynamic optimization [34] may have a place. Treatment of systolic left ventricular impairment presents additional concerns. Pharmacological therapy with catecholamines presents substantial limitations $[35,36]$, as the decreased myofilament $\mathrm{Ca}^{2+}$ sensitivity during rewarming significantly diminishes $\beta$ adrenoceptor effects. In addition, catecholamines determine elevated myocardial oxygen consumption and arrhythmogenesis. A recent study by Rungatscher and colleagues [37] tested the efficacy of levosimendan in improving myocardial dysfunction after rewarming from deep hypothermia in a rat model. Levosimendan, as a $\mathrm{Ca}^{2+}$ sensitizer, demonstrated better inotropic and lusitropic effects than epinephrine.

Glycemic homeostasis: Animal models have shown that hypothermia induces alterations in blood glucose homeostasis via several mechanisms: reduced glucose utilization [38], decreased endogenous insulin secretion [39-41], and increased resistance to exogenous insulin [42,43]. In a recently published prospective observational study dealing with glycemic homeostasis during TH after cardiac arrest (CA), Cueni-Villoz and colleagues found a significantly higher mean blood glucose concentration, blood glucose variability, and insulin dose during TH compared with the normothermia that follows passive rewarming [44]. Because the doses of adrenergic agents did not change significantly between the two steps, the authors advocated lower endogenous insulin levels and the development of insulin resistance as an explanation for the findings. The improvement in glycemic control observed during normothermia, despite lower insulin infusion, suggests that progressive recovery towards normal glycemic homeostasis occurred during rewarming. The rate of hypoglycemic episodes correlated with poor neurological outcome and was similar during $\mathrm{TH}(8 \%)$ and normothermia $(7.5 \%)$, but more frequently in patients who presented with higher blood glucose variability during $\mathrm{TH}$. These data highlight the importance of progressive tapering of insulin doses to avoid hypoglycemia during passive rewarming from $\mathrm{TH}$ after $\mathrm{CA}$, especially for patients who exhibited abrupt glucose shifts during $\mathrm{TH}$.

The importance of glycemic control is further outlined in a recent work by Smith and colleagues. The alteration of blood glucose homeostasis is associated with increased ICU morbidity and poor outcome [45]. Passive rewarming from $\mathrm{TH}$ increases insulin sensitivity, but active rewarming from cardiopulmonary bypass decreases it. In both settings, rewarming is characterized by a dynamic insulin/glucose ratio; glucose should be checked frequently and insulin requirements promptly adapted to achieve optimal glycemic control.

Electrolytes: Mild hypothermia shifts potassium inside the cells and predisposes the patient to hypokalemia, as well as hypocalcemia, hypomagnesemia, and hypophosphatemia. During rewarming, rebound increases in these electrolytes (particularly potassium) may occur, especially if they were replaced excessively during the cooling period [46]. Hyperkalemia can be prevented by slow and controlled rewarming, allowing the kidney to excrete the excess potassium. In patients with severe oliguria or anuria, renal replacement therapy should be started before rewarming to avoid hyperkalemia.

Systemic inflammation: TH has been shown to suppress ischemia-induced cerebral and systemic inflammation after traumatic brain injury (TBI) in preclinical [47-51] and clinical settings [16]. Following CA and reperfusion, $\mathrm{TH}$ is the only effective therapy for increasing survival and decreasing morbidity [52], probably by impairing harmful inflammatory reactions, which characterize systemic ischemia-reperfusion syndrome [53]. In a recent study, Bisschops and colleagues measured the kinetics of inflammatory mediators during TH and rewarming after CA [16]. Proinflammatory IL-6 increased during the TH phase, but values were surprisingly lower after rewarming. Anti-inflammatory IL-10 and IL-1RA did not significantly change over time. Complement and adhesion molecules, an index of endothelial activation, were elevated at admission, fell to low values during $\mathrm{TH}$, and increased again after rewarming, confirming the hypothesis that inflammatory processes reactivate with the increase in temperature [17]. Interestingly, no significant differences were found between artery and jugular samples, confirming that the ischemia-reperfusion phenomenon is not confined to the brain, but affects the whole organism.

Fast rewarming rates have been shown to predict worse outcomes in animal models $[49,54,55]$ due to the rapid reactivation of the inflammatory processes that were set-off by TH. Even if experimental evidence shows the advantages of controlled rewarming, additional clinical studies are needed to determine the optimal rewarming rate and strategy. Several drugs have also been tested recently in cell culture, tissue, and animal models to check their ability to mitigate the detrimental effects of rewarming. Data from Schmitt and colleagues suggest that pretreatment with methylprednisolone increases cerebral cell survival after deep hypothermia [50], but also suppresses important neuroprotective and regenerative processes induced by the proinflammatory cytokine IL-6. Diestel and colleagues focused on endothelial cells [56], which maintain systemic inflammation via cytokine production. Only combined pretreatment with methylprednisolone and tacrolimus inhibited IL- 6 secretion. A specific p38 inhibitor was demonstrated to downregulate the unwanted release of IL- 6 after cooling and rewarming most effectively. In a rat model of intestinal ischemia, gradual rewarming and administration of dexamethasone improved [48] survival and attenuated ALI after intestinal ischemia/reperfusion injury treated with TH in rats. Alva and colleagues [15] found that metabolic acidosis induced by rewarming was prevented by fructose 1,6-biphosphate ( $\mathrm{F} 1,6 \mathrm{BP})$ administration in rats. $\mathrm{F} 1,6 \mathrm{BP}$ also protected against oxidative stress induced after rewarming by decreasing lipid peroxidation in the plasma and potentiating antioxidant enzyme activities in erythrocytes. These results may be due to an increase in plasma nitric oxide and leukocytosis after the F1,6BP bolus. Other preclinical studies dealing with the anti-inflammatory safety and efficacy of these drugs are needed before a clinical study can start.

Infections: The most described infectious complication during $\mathrm{TH}$ is pneumonia. As most of the pneumonia diagnoses are made during rewarming or after achieving normothermia [57,58], several authors have claimed that rewarming itself should be considered a high risk for infection. However, as the studies cited above adopted TH for 24 hours and achieved normothermia in the following 12 to 24 hours, the occurrence of pneumonia diagnosis during or after rewarming could also be due to latency from inoculation to the clinical manifestation of infection. Moreover, as hypothermia causes an impaired inflammatory response, clinical signs of infection leading to the diagnosis may be fully detectable only after reactivation of the immune system during or after rewarming. Whether more gradual controlled rewarming can reduce the frequency of pneumonia is unclear. In a small case series [59], even very slow controlled rewarming $\left(0.1^{\circ} \mathrm{C} / \mathrm{hour}\right)$ was associated with a high frequency of pulmonary infection, perhaps because slow rewarming prolongs the total duration of hypothermia.

Coagulation: Mild platelet dysfunction occurs at temperatures $<35^{\circ} \mathrm{C}$, and some inhibition of the coagulation cascade develops at temperatures $<33^{\circ} \mathrm{C}$. In TH after TBI [58] and stroke [57], the platelet count can also decrease, which persists during and after rewarming. In neonatal cold injury, death occurring during rewarming has been attributed to massive thrombosis from platelet hyperaggregation [60].

All of these data suggest that, in the clinical setting, attention must be paid to rewarming rates and attaining the target temperature to assure the optimal effects of hypothermia. The rewarming rate is an important variable; slower rewarming rates should be routinely employed to avoid systemic side effects.

\section{References}

1. Wong KC: Physiology and pharmacology of hypothermia. West J Med 1983, 138:227-232.

2. Bacher A, llievich UM, Fitzgerald R, Ihra G, Spiss CK: Changes in oxygenation variables during progressive hypothermia in anesthetized patients. J Neurosurg Anesthesiol 1997, 9:205-210.

3. Michenfelder JD, Theye RA: Hypothermia: effect on canine brain and whole-body metabolism. Anesthesiology 1968, 29:1107-1112.

4. Lubarsky DA, Smith LR, Sladen RN, Mault JR, Reed RL: Defining the relationship of oxygen delivery and consumption: use of biologic system models. J Surg Res 1995, 58:503-508.

5. Hegnauer AH, Flynn J, D'Amato H: Cardiac physiology in dog during rewarming from deep hypothermia. Am J Physiol 1951, 167:69-75.

6. Bigelow WC: Methods for inducing hypothermia and rewarming. Ann N Y Acad Sci 1959, 80:522-532.

7. Dixon SR, Whitbourn RJ, Dae MW, Grube E, Sherman W, Schaer GL, et al: Induction of mild systemic hypothermia with endovascular cooling during primary percutaneous coronary intervention for acute myocardial infarction. J Am Coll Cardiol 2002, 40:1928-1934.

8. Mottillo S, Sharma K, Eisenberg MJ: Therapeutic hypothermia in acute myocardial infarction: a systematic review. Can J Cardiol 2011, 27:555-561. 
9. Han YS, Tveita T, Prakash YS, Sieck GC: Mechanisms underlying hypothermia-induced cardiac contractile dysfunction. Am J Physiol Heart Circ Physiol 2010, 298:H890-H897.

10. Tveita T, Myklebust $R$, Ytrehus $K$ : Changes in myocardial ultrastructure induced by cooling as well as rewarming. Res Exp Med (Berl) 1998, 197:243-254

11. Blair E, Montgomery AV, Swan H: Posthypothermic circulatory failure. I. Physiologic observations on the circulation. Circulation 1956, 13:909-915.

12. Morray JP, Pavlin EG: Oxygen delivery and consumption during hypothermia and rewarming in the dog. Anesthesiology 1990, 72:510-516.

13. Steen PA, Milde JH, Michenfelder JD: The detrimental effects of prolonged hypothermia and rewarming in the dog. Anesthesiology 1980, 52:224-230.

14. Talaei F, Bouma HR, Van der Graaf AC, Strijkstra AM, Schmidt M, Henning RH: Serotonin and dopamine protect from hypothermia/rewarming damage through the CBS/H2S pathway. PLoS One 2011, 6:e22568.

15. Alva N, Carbonell T, Roig T, Bermudez J, Palomeque J: Fructose 1,6 biphosphate administration to rats prevents metabolic acidosis and oxidative stress induced by deep hypothermia and rewarming. Eur $J$ Pharmacol 2011, 659:259-264.

16. Bisschops LL, Hoedemaekers CW, Mollnes TE, van der Hoeven JG: Rewarming after hypothermia after cardiac arrest shifts the inflammatory balance. Crit Care Med 2012, 40:1136-1142.

17. Hildebrand $F$, van Griensven M, Giannoudis P, Luerig A, Harwood $P$, Harms $O$, et al: Effects of hypothermia and re-warming on the inflammatory response in a murine multiple hit model of trauma. Cytokine 2005, 31:382-393.

18. Horvath SM, Spurr GB, Hutt BK, Hamilton LH: Metabolic cost of shivering. J Appl Physiol 1956, 8:595-602.

19. Bay J, Nunn JF, Prys-Roberts $\mathrm{C}$ : Factors influencing arterial $\mathrm{PO}_{2}$ during recovery from anaesthesia. $\mathrm{Br} J$ Anaesth 1968, 40:398-407.

20. Ralley FE, Wynands JE, Ramsay JG, Carli F, MacSullivan R: The effects of shivering on oxygen consumption and carbon dioxide production in patients rewarming from hypothermic cardiopulmonary bypass. Can J Anaesth 1988, 35:332-337.

21. Cain SM, Bradley WE: Critical $\mathrm{O}_{2}$ transport values at lowered body temperatures in rats. J Appl Physiol 1983, 55:1713-1717.

22. Schumacker PT, Rowland J, Saltz S, Nelson DP, Wood LD: Effects of hyperthermia and hypothermia on oxygen extraction by tissues during hypovolemia. J Appl Physiol 1987, 63:1246-1252.

23. Leducq N, Delmas-Beauvieux MC, Bourdel-Marchasson I, Dufour S, Gallis JL, Canioni $P$, et al: Mitochondrial permeability transition during hypothermic to normothermic reperfusion in rat liver demonstrated by the protective effect of cyclosporin A. Biochem J 1998, 336(Pt 2):501-506.

24. Kondratiev TV, Flemming K, Myhre ES, Sovershaev MA, Tveita T: Is oxygen supply a limiting factor for survival during rewarming from profound hypothermia? Am J Physiol Heart Circ Physiol 2006, 291:H441-H450.

25. Polderman $\mathrm{KH}$, Callaghan J: Equipment review: cooling catheters to induce therapeutic hypothermia? Crit Care 2006, 10:234.

26. Thoresen M, Whitelaw A: Cardiovascular changes during mild therapeutic hypothermia and rewarming in infants with hypoxic-ischemic encephalopathy. Pediatrics 2000, 106(1 Pt 1):92-99.

27. Saleh M, Barr TM: The impact of slow rewarming on inotropy, tissue metabolism, and 'after drop' of body temperature in pediatric patients. J Extra Corpor Technol 2005, 37:173-179.

28. Hanhela R, Mustonen A, Korhonen I, Salomaki T: The effects of two rewarming strategies on heat balance and metabolism after coronary artery bypass surgery with moderate hypothermia. Acta Anaesthesiol Scand 1999, 43:979-988.

29. Michenfelder JD, Uihlein A, Daw EF, Theye RA: Moderate hypothermia in man: haemodynamic and metabolic effects. Br J Anaesth 1965, 37:738-745.

30. Rodriguez JL, Weissman C, Damask MC, Askanazi J, Hyman Al, Kinney JM: Physiologic requirements during rewarming: suppression of the shivering response. Crit Care Med 1983, 11:490-497.

31. Zwischenberger JB, Kirsh MM, Dechert RE, Arnold DK, Bartlett RH: Suppression of shivering decreases oxygen consumption and improves hemodynamic stability during postoperative rewarming. Ann Thorac Surg 1987, 43:428-431.

32. Badjatia N, Strongilis E, Gordon E, Prescutti M, Fernandez L, Fernandez A, et al: Metabolic impact of shivering during therapeutic temperature modulation: the Bedside Shivering Assessment Scale. Stroke 2008, 39:3242-3247.
33. Macintyre PE, Pavlin EG, Dwersteg JF: Effect of meperidine on oxygen consumption, carbon dioxide production, and respiratory gas exchange in postanesthesia shivering. Anesth Analg 1987, 66:751-755.

34. Jones AE, Shapiro NI, Kilgannon JH, Trzeciak S: Goal-directed hemodynamic optimization in the post-cardiac arrest syndrome: a systematic review. Resuscitation 2008, 77:26-29.

35. Tveita T, Sieck GC: The physiologic responses to epinephrine during cooling and after rewarming in vivo. Crit Care 2011, 15:R225.

36. Weiss SJ, Muniz A, Ernst AA, Lippton HL: The physiological response to norepinephrine during hypothermia and rewarming. Resuscitation 1998, 39:189-195.

37. Rungatscher A, Linardi D, Tessari M, Menon T, Luciani GB, Mazzucco A, et al: Levosimendan is superior to epinephrine in improving myocardial function after cardiopulmonary bypass with deep hypothermic circulatory arrest in rats. J Thorac Cardiovasc Surg 2012, 143:209-214.

38. Escolar JC, Hoo-Paris R, Castex C, Sutter BC: Effect of low temperatures on glucose-induced insulin secretion and glucose metabolism in isolated pancreatic islets of the rat. J Endocrinol 1990, 125:45-51.

39. Blackard WG, Nelson NC, Labat JA: Insulin secretion in hypothermic dogs. Am J Physiol 1967, 212:1185-1187.

40. Escolar JC, Hoo-Paris R, Castex C, Sutter BC: Effect of low temperatures on glucose-induced insulin secretion and ionic fluxes in rat pancreatic islets. J Endocrinol 1987, 115:225-231.

41. Sasaki Y, Takahashi H, Aso H, Ohneda A, Weekes TE: Effects of cold exposure on insulin and glucagon secretion in sheep. Endocrinology 1982, 111:2070-2076.

42. Helman A, Gilbert M, Pfister-Lemaire N, Reach G, Assan R: Glucagon and insulin secretion and their biological activities in hypothermic rats. Endocrinology 1984, 115:1722-1728.

43. Torlinska T, Perz M, Madry E, Hryniewiecki T, Nowak KW, Mackowiak P: Effect of hypothermia on insulin-receptor interaction in different rat tissues. Physiol Res 2002, 51:261-266.

44. Cueni-Villoz N, Devigili A, Delodder F, Cianferoni S, Feihl F, Rossetti AO, et al: Increased blood glucose variability during therapeutic hypothermia and outcome after cardiac arrest. Crit Care Med 2011, 39:2225-2231.

45. Smith FG, Sheehy AM, Vincent JL, Coursin DB: Critical illness-induced dysglycaemia: diabetes and beyond. Crit Care 2010, 14:327.

46. Polderman KH, Peerdeman SM, Girbes AR: Hypophosphatemia and hypomagnesemia induced by cooling in patients with severe head injury. J Neurosurg 2001, 94:697-705.

47. Alam HB, Rhee $P$, Honma $K$, Chen $H$, Ayuste EC, Lin $T$, et al: Does the rate of rewarming from profound hypothermic arrest influence the outcome in a swine model of lethal hemorrhage? J Trauma 2006, 60:134-146.

48. Kim K, Jo YH, Rhee JE, Kim TY, Lee JH, Kwon WY, et al: Effect of speed of rewarming and administration of anti-inflammatory or anti-oxidant agents on acute lung injury in an intestinal ischemia model treated with therapeutic hypothermia. Resuscitation 2010, 81:100-105.

49. Gordan ML, Kellermann K, Blobner M, Nollert G, Kochs EF, Jungwirth B: Fast rewarming after deep hypothermic circulatory arrest in rats impairs histologic outcome and increases NFkappaB expression in the brain. Perfusion 2010, 25:349-354.

50. Schmitt KR, Kern C, Berger F, Ullrich O, Hendrix S, Abdul-Khaliq H: Methylprednisolone attenuates hypothermia- and rewarming-induced cytotoxicity and IL-6 release in isolated primary astrocytes, neurons and BV-2 microglia cells. Neurosci Lett 2006, 404:309-314.

51. Vaagenes P, Gundersen Y, Opstad PK: Rapid rewarming after mild hypothermia accentuates the inflammatory response after acute volume controlled haemorrhage in spontaneously breathing rats. Resuscitation 2003, 58:103-112.

52. Bernard SA, Buist M: Induced hypothermia in critical care medicine: a review. Crit Care Med 2003, 31:2041-2051.

53. Scumpia PO, Sarcia PJ, Kelly KM, DeMarco VG, Skimming JW: Hypothermia induces anti-inflammatory cytokines and inhibits nitric oxide and myeloperoxidase-mediated damage in the hearts of endotoxemic rats. Chest 2004, 125:1483-1491

54. Maxwell WL, Watson A, Queen R, Conway B, Russell D, Neilson M, et al: Slow, medium, or fast re-warming following post-traumatic hypothermia therapy? An ultrastructural perspective. J Neurotrauma 2005, 22:873-884.

55. Berger C, Xia F, Kohrmann M, Schwab S: Hypothermia in acute stroke slow versus fast rewarming an experimental study in rats. Exp Neurol 2007, 204:131-137. 
56. Diestel A, Roessler J, Pohl-Schickinger A, Koster A, Drescher C, Berger F, et al: Specific p38 inhibition in stimulated endothelial cells: a possible new anti-inflammatory strategy after hypothermia and rewarming. Vascul Pharmacol 2009, 51:246-252.

57. Schwab S, Schwarz S, Spranger M, Keller E, Bertram M, Hacke W: Moderate hypothermia in the treatment of patients with severe middle cerebral artery infarction. Stroke 1998, 29:2461-2466.

58. Metz C, Holzschuh M, Bein T, Woertgen C, Frey A, Frey l, et al: Moderate hypothermia in patients with severe head injury: cerebral and extracerebral effects. J Neurosurg 1996, 85:533-541.

59. Steiner T, Friede T, Aschoff A, Schellinger PD, Schwab S, Hacke W: Effect and feasibility of controlled rewarming after moderate hypothermia in stroke patients with malignant infarction of the middle cerebral artery. Stroke 2001, 32:2833-2835.

60. Diephuis JC, Balt J, van Dijk D, Moons KG, Knape JT: Effect of rewarming speed during hypothermic cardiopulmonary bypass on cerebral pressure-flow relation. Acta Anaesthesio/ Scand 2002, 46:283-288.

\section{A26}

Hypothermia in refractory status epilepticus

Andrea O Rossetti

Service de Neurologie, CHUV-BH07, Lausanne, Switzerland

Critical Care 2012, 16(Suppl 2):A26

Introduction: Status epilepticus (SE) is a neurological emergency with potentially important mortality and morbidity. After refractoriness to general anesthetics, several pharmacological and nonpharmacological options have been described more or less anecdotally. In this context, and despite animal data supporting neuroprotective actions of brain hypothermia and showing its efficacy in SE models, hypothermia targeting a core temperature of about $33^{\circ} \mathrm{C}$ for at least 24 hours together with pharmacological sedation has been scarcely reported in adults and children. It seems that this approach rarely allows a sustained control of $\mathrm{SE}$, as seizures tend to recur in normothermic conditions. Conversely, hypothermia has a high evidence level and is increasingly used in postanoxic encephalopathy, both in newborns and adults. Due to the thin available clinical evidence, prospective studies are needed to define the value of hypothermia in SE.

Refractory status epilepticus and its treatment: SE represents the second most frequent neurological emergency after acute stroke, and bears significant risks of morbidity and mortality [1]. SE persisting despite adequate doses of benzodiazepines and at least one antiepileptic drug (AED) is considered refractory (RSE) [2,3]; this develops in 23 to $43 \%$ of patients with SE. RSE is associated with acute, severe and potentially fatal underlying etiologies, such as encephalitis, large stroke, or rapidly progressive primary brain tumors, and may be accompanied by coma; these factors, together with increasing age, represent the most important outcome predictors [1].

After securing pulmonary and cardiac functions, intravenous administration of a sequence of three groups of drugs represents the mainstay of management [4]: benzodiazepines (the only clearly evidence-based step); classical antiepileptic drugs (AED, mostly phenytoin, valproate, or levetiracetam); and general anaesthetics for RSE. Among anaesthetics, midazolam, propofol, or barbiturates represents the most popular agents, without any hard evidence favoring one specific compound [1]. Anesthetic treatment may lead to various complications (infections, metabolic disturbances, ileus, neuropathy, myopathy, thromboembolic events) [5]; it is therefore necessary to balance these risks with the benefit of rapid seizure control. Generalized convulsive RSE should be treated rapidly with general anesthetics, given the danger of systemic and neurological injury with ongoing convulsions; conversely, nonconvulsive SE without marked consciousness impairment can be approached more conservatively, as these forms are probably not associated with the same risk of injury $[1,2,6]$.

RSE that proves refractory to a first course of general anesthetics implies a (repeated) careful search of the underlying etiology. This condition may be managed in several ways, which mostly rely on small series or case reports $[1,7]$. Briefly, pharmacological options may include further use of anesthetics (the three aforementioned, ketamine, isofluorane), other AED (for example, topiramate, lacosamide), or ketogenic diet. Reported nonpharmacological approaches span from resective surgery, through vagus-nerve, electroconvulsive or transcranial magnetic stimulations, to mild therapeutic hypothermia (TH).
While the benefits of hypothermia on patients with head injury were already described by Hippocrates [8], TH enjoys an only evidence-based status in the setting of adult and pediatric postanoxic encephalopathy, and reduction of intracranial pressure [9]. Its indication for the treatment of other acute brain disorders, including SE and traumatic brain injury, is essentially anecdotic.

Animal data on hypothermia: Low brain temperature exerts beneficial effects on the cascades involved in acute cerebral injuries; several seminal studies have been recently reviewed $[9,10]$. Hypothermia reduces brain metabolism and ATP consumption, and leads to decrease of glutamate release, free radicals, oxidative stress, mitochondrial dysfunction and calcium overload. Conversely, brain-derived neurotrophic factor increases; as a result, apoptosis is inhibited. Furthermore, hypothermia reduces reperfusion injury, permeability of the blood-brain barrier, and inflammatory reactions. Of note, most of these mechanisms are involved in the pathophysiology of SE leading to neuronal injury [11]. Various rodent models of SE support the neuroprotective effects of $\mathrm{TH}$, showing (with concomitant benzodiazepines) a reduction of seizure severity in SE triggered by electrical stimulation of limbic structures [12], and mitigation of seizures, brain edema, and cognitive deficits in kainate-induced SE [13]. Temperature lowering before pilocarpine injections (a proconvulsant) protects against SE and apopotosis [14]. However, TH has received very little attention in clinical settings.

Experience in patients with refractory status epilepticus: More than two decades ago, three children with generalized SE were successfully treated with $\mathrm{TH}\left(30\right.$ to $\left.31^{\circ} \mathrm{C}\right)$ and thiopental [15]. Four adults with SE triggered by limbic encephalitis (two patients), hepatic encephalopathy (one patient) and of unknown origin (one patient) were treated with $\mathrm{TH}\left(31\right.$ to $\left.35^{\circ} \mathrm{C}\right)$ coadministered with midazolam; SE was controlled in all, but two patients later died [16]. Shivering was managed by neuromuscular blockade; vein thrombosis and pulmonary embolism were the reported side effects. Another adult with cryptogenic SE was treated with $\mathrm{TH}\left(34^{\circ} \mathrm{C}\right)$ and thiopental, but she developed paralytic ileus requiring emergency intestinal resection; she survived after further treatment of her RSE [5]. Ongoing seizures in an infant with a severe developmental disorder were controlled by $\mathrm{TH}$ at 35 to $36^{\circ} \mathrm{C}$, together with ketamine; subsequently, hemispherectomy had to be carried out [17]. Finally, an abstract in Japanese describes an improved functional outcome in 12 children treated with $\mathrm{TH}$ (34 to $36^{\circ} \mathrm{C}$ ) and general anesthesia for febrile SE, as compared with 16 treated with conventional therapy, in a retrospective assessment [18]. Reported $\mathrm{TH}$ durations are variable, between 20 hours and several days. These case studies suggest that hypothermia may contribute to seizure control. However, its efficacy seems to be only transient: seizures tend to recur in normothermia. TH may thus represent an option in severely refractory SE, but rather to gain some time as to definitively control seizures.

Recently, it has been recognized that postanoxic SE, even with early myoclonus, does not imply an invariably dismal outcome. It seems that SE occurring during $\mathrm{TH}$, mostly as a seizure suppression EEG pattern, does reflect an extremely severe brain damage, and patients are extremely unlikely to survive $[19,20]$; conversely, SE arising after return of normothermia and in presence of a reactive EEG background, and preservation of brainstem reflexes and early cortical somatosensory evoked potentials, may be successfully treated with the usual therapeutic armamentarium; those cases represent at most $10 \%$ of patients with postanoxic SE, and a good functional outcome can be reached [21]. This actually suggests that TH (with moderate midazolam or propofol doses) can be sufficient to transitorily control benign postanoxic SE (corroborating its antiepileptic properties), while it does not prevent a poor outcome in more severe forms.

Conclusion: As there is a lack of clinical evidence, mild $\mathrm{TH}\left(32\right.$ to $36^{\circ} \mathrm{C}$ ) may represent a therapeutic option for RSE, albeit on a patient by patient basis. Barbiturates should be avoided because of the risk of paralytic ileus (thus favoring midazolam or propofol), and mild hypothermia should be administered for 24 to 48 hours. Repeated controls of cardiovascular indices, coagulation parameters and lactate (metabolic acidosis following severe infections or intestinal necrosis), and clinical surveillance (vein thrombosis) are mandatory. A well-designed, prospective trial appears necessary to assess the exact role of $\mathrm{TH}$ in $\mathrm{SE}$.

\section{References}

1. Rossetti AO, Lowenstein DH: Management of refractory status epilepticus in adults: still more questions than answers. Lancet Neurol 2011, 10:922-930.

2. Holtkamp M: Treatment strategies for refractory status epilepticus. Curr Opin Crit Care 2011, 17:94-100. 
3. Novy J, Logroscino G, RossettiAO : Refractory status epilepticus: a prospective observational study. Epilepsia 2010, 51:251-256.

4. Meierkord $H$, et al: EFNS guideline on the management of status epilepticus in adults. Eur J Neurol 2010, 17:348-355.

5. Cereda C, Berger MM, Rossetti AO: Bowel ischemia: a rare complication of thiopental treatment for status epilepticus. Neurocrit Care 2009, 10:355-358.

6. Shorvon S: The treatment of status epilepticus. Curr Opin Neurol 2011, 24:165-170.

7. Shorvon S, Ferlisi M: The treatment of super-refractory status epilepticus: a critical review of available therapies and a clinical treatment protocol. Brain 2011, 134(Pt 10):2802-2818

8. Harris $\mathrm{OA}$, et al: The role of hypothermia in the management of severe brain injury: a meta-analysis. Arch Neurol 2002, 59:1077-1083.

9. Polderman $\mathrm{KH}$ : Induced hypothermia and fever control for prevention and treatment of neurological injuries. Lancet 2008, 371:1955-1969.

10. Holzer M: Targeted temperature management for comatose survivors of cardiac arrest. N Engl J Med 2010, 363:1256-1264.

11. Chen JW, Wasterlain CG: Status epilepticus: pathophysiology and management in adults. Lancet Neurol 2006, 5:246-256.

12. Schmitt FC, et al: Anticonvulsant properties of hypothermia in experimental status epilepticus. Neurobiol Dis 2006, 23:689-696.

13. Wang Y, Liu PP, Li LY, Zhang HM, Li T: Hypothermia reduces brain edema, spontaneous recurrent seizure attack, and learning memory deficits in the kainic acid treated rats. CNS Neurosci Ther 2011, 17:271-280.

14. Yu L, Zhou Y, Chen W, Wang Y: Mild hypothermia pretreatment protects against pilocarpine-induced status epilepticus and neuronalapoptosis in immature rats. Neuropathology 2011, 31:144-151.

15. Orlowski JP, et al: Hypothermia and barbiturate coma for refractory status epilepticus. Crit Care Med 1984, 12:367-372.

16. Corry JJ, et al: Hypothermia for refractory status epilepticus. Neurocrit Care 2008, 9:189-197.

17. Elting JW, Naalt J, Fock JM: Mild hypothermia for refractory focal status epilepticus in an infant with hemimegalencephaly. Eur J Paediatr Neurol 2010, 14:452-455.

18. Nakagawa $T$, et al: [Induced hypothermia/normothermia with general anesthesia prevents neurological damage in febrile refractory status epilepticus in children]. No To Hattatsu 2011, 43:459-463.

19. Rossetti $\mathrm{AO}$, et al: Prognostic value of continuous EEG monitoring during therapeutic hypothermia after cardiac arrest. Crit Care 2010, 14:R173.

20. Thenayan EA, et al: Electroencephalogram for prognosis after cardiac arrest. J Crit Care 2010, 25:300-304.

21. Rossetti $A O$, et al: Predictors of awakening from postanoxic status epilepticus after therapeutic hypothermia. Neurology 2009, 72:744-749.

\section{A27}

Hypothermia and advanced neuromonitoring

Raimund Helbok*, Alois Schiefecker, Marlene Fischer, Anelia Dietmann, Erich Schmutzhard

Department of Neurology, Neurocritical Care Unit, Medical University Hospital Innsbruck, Austria

Critical Care 2012, 16(Suppl 2):A27

Therapeutic hypothermia (TH) improves short-term neurologic outcome and reduces mortality after cardiac arrest (CA) [1]. Neuroprotective mechanisms comprise reduced cerebral metabolic demand [2,3], decreased excitotoxicity [4-7], cell membrane stabilization [8,9], inhibition of spreading depolarizations [10] and cytokine release [11], and preservation of cerebral autoregulation. This concept of neuroprotection led to clinical trials using TH for the prevention of secondary brain injury in patients with traumatic brain injury, subarachnoid hemorrhage (SAH) and ischemic stroke; however, they failed to show any benefit in clinical outcomes [12-15]. This may reflect our lack of understanding of the exact pathophysiologic processes induced by $\mathrm{TH}$ and the potential harm through hypothermia and rewarming injury [16].

Advanced neuromonitoring techniques allow online measurement of brain metabolism, cerebral autoregulation, brain tissue oxygenation, and cerebral blood flow (CBF) and provide information about brain function, energy supply and demand [17-19]. Here we will summarize current knowledge about the effects of TH on brain homeostasis after acute brain injury using advanced neuromonitoring techniques and call for more observational trials investigating pathophysiologic mechanisms during $\mathrm{TH}$ and rewarming to maximize the benefits of this emerging therapeutic modality.

TH effectively decreases intracranial pressure (ICP) by up to $10 \mathrm{mmHg}$ $[12,20-22]$, whereby mild hypothermia $\left(35^{\circ} \mathrm{C}\right)$ seems to be as effective as $33^{\circ} \mathrm{C}[21,23]$. Mechanisms include the reduction in metabolic demand and the inhibitory effect on inflammation and free radical production stabilizing the blood-brain barrier and decreasing vasogenic edema [20,24-26].

Cerebral microdialysis allows bedside monitoring of cerebral metabolic changes from the extracellular fluid of the brain [27]. Decreased energy supply, increased demand or mitochondrial dysfunction may result in brain metabolic distress and/or brain hypoglycemia (brain glucose $<0.7 \mathrm{mmol} / \mathrm{l}$ ). Cerebral microdialysis is feasible during $\mathrm{TH}$ and sensitive to detect secondary energy failure as indicated by an increase in the lactatepyruvate ratio (LPR) $[28,29]$. Therapeutic hypothermia reduces brain metabolic demand for oxygen and glucose and preserves ATP supply to the brain decreasing the risk of secondary energy failure [29-32]. Moreover, extensive cerebral lactate accumulation is inhibited by $\mathrm{TH}$, which ameliorates the deleterious effects on cell membranes and the blood-brain barrier [33]. Increased brain glucose may be found during $\mathrm{TH}$ [6]; however, an increased blood glucose variability during hypothermia has been reported [34]. This may negatively affect brain glucose as sudden decreases in systemic glucose have been associated with brain metabolic distress and worse outcome [35]. Therefore monitoring of brain glucose is important to detect brain hypoglycemia and prevent further neuronal damage during $\mathrm{TH}$ and the rewarming phase. An additional beneficial effect of hypothermia that has been extensively studied in vitro and in animal models is the reduction in excitotoxic neurotransmitter release $[4-7,28]$, thereby inhibiting nitric oxide synthesis and apoptotic pathways $[16,36]$. A decrease of extracellular glutamate was observed during $\mathrm{TH}$ after cardiac arrest and in ischemic stroke patients using cerebral microdialysis $[28,37]$. In summary, cerebral microdialysis allows monitoring of the metabolic effects of TH after acute brain injury.

Another mechanism how $\mathrm{TH}$ may reduce secondary energy failure is a decrease in oxygen consumption through diminished metabolism [2,3], resulting in increased brain tissue oxygenation $[25,38]$. Brain tissue oxygenation reflects the net effect of oxygen delivery, diffusion and consumption and can be assessed by positron emission tomography, magnetic resonance spectroscopy, near-infrared spectroscopy or by invasive $\mathrm{P}_{\mathrm{b}} \mathrm{tO}_{2}$ probes $[39,40]$. Therapeutic hypothermia below $35^{\circ} \mathrm{C}$ may impair brain tissue oxygenation through a left-shift of the oxygen dissociation curve, therefore enhancing the affinity of oxygen to hemoglobin, or by decreasing delivery of oxygen to the brain $[21,41]$. Jugular bulb oxygen saturation $\left(\mathrm{jvO}_{2}\right)$ is a global measurement of brain oxygen extraction and is increased during mild $\mathrm{TH}[31,41]$, reflecting a reduction in cerebral metabolic rate of oxygen.

Shivering is frequently observed during $\mathrm{TH}$ and may abolish the neuroprotective effect of temperature modulation through increase in metabolic demand and systemic and cerebral energy consumption [42]. A shivering-associated reduction in $\mathrm{P}_{\mathrm{b}} \mathrm{tO}_{2}$ seems to correlate with the intensity of therapeutic cooling and potentially increases the risk of brain hypoxia [43]. These results imply that the neuroprotective effect of $\mathrm{TH}$ may be most beneficial at a temperature not lower than $35^{\circ} \mathrm{C}$ and shivering should be assessed at the bedside and effectively treated by pharmacological and nonpharmacological means [42].

It is important to note that $\mathrm{CO}_{2}$ reactivity may be preserved during $\mathrm{TH}$ [44]. Therapeutic hyperventilation as $\mathrm{TH}$ is used as rescue therapy to decrease raised ICP and unintentional hypocapnia is also commonly observed in patients with acute brain injury [45], which increases the risk of brain tissue hypoxia [38]. Monitoring of brain tissue oxygen or jugular venous oxygen saturation $\left(\mathrm{jSVO}_{2}\right)$ is recommended for patients with acute traumatic brain injury, where therapeutic hyperventilation is used [46] and is important especially during $\mathrm{TH}$. Preserved cerebral autoregulation seems not to be disturbed during $\mathrm{TH}$ and early induction of hypothermia after $\mathrm{SAH}$ led to faster restoration of cerebrovascular reactivity in vivo $[6,47,48]$.

The rewarming has been considered as the vulnerable phase following $\mathrm{TH}$ $[42,49,50]$. Rapid rewarming and timing in vulnerable phases of the injured brain may abolish the neuroprotective effects of TH through ICP increase, excitotoxicity, increased metabolic demand and derangement of cerebrovascular reactivity [42,51-53]. A report of four patients treated with $\mathrm{TH}$ after CA observed an increase in LPR in all patients during rewarming 
indicating brain ischemia [28]. Slow and controlled rewarming after moderate hypothermia may prevent ICP increase and glutamate release and stabilize infarct volume [53]. Close monitoring of cerebral metabolism, $I C P, C B F$ and brain tissue oxygenation can help to define the optimal rewarming rate to avoid increases in ICP (recommended rate of $0.1^{\circ} \mathrm{C}$ ) and to early detect an imbalance in energy supply and demand.

Conclusion: In the clinical setting there is still need to further explore the best induction and maintenance method, optimal duration and targeted temperature of therapeutic hypothermia. Due to the complexity of pathophysiologic mechanisms during hypothermia and rewarming, combining different advanced monitoring techniques seems mandatory. Multimodal neuromonitoring guidance may then help to define therapeutic targets and to establish clinical protocols to maximize the benefits of this emerging therapeutic modality.

\section{References}

1. Mild therapeutic hypothermia to improve the neurologic outcome after cardiac arrest. N Engl J Med 2002, 346:549-556.

2. Rosomoff $\mathrm{HL}$, Holaday DA: Cerebral blood flow and cerebral oxygen consumption during hypothermia. Am J Physiol 1954, 179:85-88.

3. Chopp M, Knight R, Tidwell CD, Helpern JA, Brown E, Welch KM: The metabolic effects of mild hypothermia on global cerebral ischemia and recirculation in the cat: comparison to normothermia and hyperthermia. J Cereb Blood Flow Metab 1989, 9:141-148.

4. Busto R, Globus MY, Dietrich WD, Martinez E, Valdes I, Ginsberg MD: Effect of mild hypothermia on ischemia-induced release of neurotransmitters and free fatty acids in rat brain. Stroke 1989, 20:904-910.

5. Winfree CJ, Baker CJ, Connolly ES, Fiore AJ, Solomon RA: Mild hypothermia reduces penumbral glutamate levels in the rat permanent focal cerebral ischemia model. Neurosurgery 1996, 38:1216-1222.

6. Schubert GA, Poli S, Mendelowitsch A, Schilling L, Thome C: Hypothermia reduces early hypoperfusion and metabolic alterations during the acute phase of massive subarachnoid hemorrhage: a laser-Doppler-flowmetry and microdialysis study in rats. J Neurotrauma 2008, 25:539-548.

7. Shima H, Fujisawa H, Suehiro E, Uetsuka S, Maekawa T, Suzuki M: Mild hypothermia inhibits exogenous glutamate-induced increases in nitric oxide synthesis. J Neurotrauma 2003, 20:1179-1187.

8. Fischer S, Renz D, Wiesnet M, Schaper W, Karliczek GF: Hypothermia abolishes hypoxia-induced hyperpermeability in brain microvessel endothelial cells. Brain Res Mol Brain Res 1999, 74:135-144.

9. Jiang JY, Lyeth BG, Kapasi MZ, Jenkins LW, Povlishock JT: Moderate hypothermia reduces blood-brain barrier disruption following traumatic brain injury in the rat. Acta Neuropathol 1992, 84:495-500.

10. Ueda M, Watanabe N, Ushikubo Y, Kasai K, Tsuzuki T, Aoki K, Yamazaki Y, Samejima H: [The effect of hypothermia on CSD propagation in rats]. No Shinkei Geka 1997, 25:523-528.

11. Marion DW, Penrod LE, Kelsey SF, Obrist WD, Kochanek PM, Palmer AM, Wisniewski SR, DeKosky ST: Treatment of traumatic brain injury with moderate hypothermia. N Engl J Med 1997, 336:540-546.

12. Clifton GL, Miller ER, Choi SC, Levin HS, McCauley S, Smith KR, Muizelaar JP, Wagner FC, Marion DW, Luerssen TG, et al: Lack of effect of induction of hypothermia after acute brain injury. N Engl I Med 2001, 344:556-563.

13. Linares G, Mayer SA: Hypothermia for the treatment of ischemic and hemorrhagic stroke. Crit Care Med 2009, 37:S243-S249.

14. Todd MM, Hindman BJ, Clarke WR, Torner JC: Mild intraoperative hypothermia during surgery for intracranial aneurysm. N Engl J Med 2005, 352:135-145.

15. Hutchison JS, Ward RE, Lacroix J, Hebert PC, Barnes MA, Bohn DJ, Dirks PB, Doucette $S$, Fergusson D, Gottesman R, et al: Hypothermia therapy after traumatic brain injury in children. N Engl J Med 2008, 358:2447-2456.

16. Warren DE, Bickler PE, Clark JP, Gregersen M, Brosnan H, McKleroy W, Gabatto P: Hypothermia and rewarming injury in hippocampal neurons involve intracellular $\mathrm{Ca}^{2+}$ and glutamate excitotoxicity. Neuroscience 2012, 207:316-325.

17. Stuart RM, Schmidt M, Kurtz P, Waziri A, Helbok R, Mayer SA, Lee K, Badjatia N, Hirsch L, Connolly ES, Claassen J: Intracranial multimodal monitoring for acute brain injury: a single institution review of current practices. Neurocrit Care 2010, 12:188-198.

18. Stover JF: Actual evidence for neuromonitoring-guided intensive care following severe traumatic brain injury. Swiss Med Wkly 2011, 141 W13245.

19. Vajkoczy P, Roth H, Horn P, Lucke T, Thome C, Hubner U, Martin GT, Zappletal C, Klar E, Schilling L, Schmiedek P: Continuous monitoring of regional cerebral blood flow: experimental and clinical validation of a novel thermal diffusion microprobe. J Neurosurg 2000, 93:265-274.

20. Schubert GA, Poli S, Schilling L, Heiland S, Thome C: Hypothermia reduces cytotoxic edema and metabolic alterations during the acute phase of massive SAH: a diffusion-weighted imaging and spectroscopy study in rats. J Neurotrauma 2008, 25:841-852.

21. Tokutomi T, Morimoto K, Miyagi T, Yamaguchi S, Ishikawa K, Shigemori M: Optimal temperature for the management of severe traumatic brain injury: effect of hypothermia on intracranial pressure, systemic and intracranial hemodynamics, and metabolism. Neurosurgery 2003, 52:102-111, discussion 111-112.

22. Schreckinger M, Marion DW: Contemporary management of traumatic intracranial hypertension: is there a role for therapeutic hypothermia? Neurocrit Care 2009, 11:427-436.

23. Tokutomi T, Miyagi T, Takeuchi Y, Karukaya T, Katsuki H, Shigemori M: Effect of $35^{\circ} \mathrm{C}$ hypothermia on intracranial pressure and clinical outcome in patients with severe traumatic brain injury. J Trauma 2009, 66:166-173.

24. Shiozaki T, Sugimoto $H$, Taneda M, Yoshida $H$, Iwai A, Yoshioka T, Sugimoto T: Effect of mild hypothermia on uncontrollable intracranial hypertension after severe head injury. J Neurosurg 1993, 79:363-368.

25. Soukup J, Zauner A, Doppenberg EM, Menzel M, Gilman C, Young HF, Bullock R: The importance of brain temperature in patients after severe head injury: relationship to intracranial pressure, cerebral perfusion pressure, cerebral blood flow, and outcome. J Neurotrauma 2002, 19:559-571.

26. Polderman $\mathrm{KH}$ : Mechanisms of action, physiological effects, and complications of hypothermia. Crit Care Med 2009, 37:S186-S202.

27. Hillered L, Persson L, Nilsson P, Ronne-Engstrom E, Enblad P: Continuous monitoring of cerebral metabolism in traumatic brain injury: a focus on cerebral microdialysis. Curr Opin Crit Care 2006, 12:112-118.

28. Nordmark J, Rubertsson S, Mortberg E, Nilsson P, Enblad P: Intracerebral monitoring in comatose patients treated with hypothermia after a cardiac arrest. Acta Anaesthesiol Scand 2009, 53:289-298.

29. Berger C, Kiening K, Schwab S: Neurochemical monitoring of therapeutic effects in large human MCA infarction. Neurocrit Care 2008, 9:352-356.

30. Steen PA, Newberg L, Milde JH, Michenfelder JD: Hypothermia and barbiturates: individual and combined effects on canine cerebral oxygen consumption. Anesthesiology 1983, 58:527-532.

31. Nordmark J, Enblad P, Rubertsson S: Cerebral energy failure following experimental cardiac arrest Hypothermia treatment reduces secondary lactate/pyruvate-ratio increase. Resuscitation 2009, 80:573-579.

32. Erecinska M, Thoresen M, Silver IA: Effects of hypothermia on energy metabolism in Mammalian central nervous system. J Cereb Blood Flow Metab 2003, 23:513-530.

33. Jiang JY, Liang YM, Luo QZ, Zhu C: Effect of mild hypothermia on brain dialysate lactate after fluid percussion brain injury in rodents. Neurosurgeny 2004, 54:713-717, discussion 717-718.

34. Cueni-Villoz N, Devigili A, Delodder F, Cianferoni S, Feihl F, Rossetti AO, Eggimann P, Vincent IL, Taccone FS, Oddo M: Increased blood glucose variability during therapeutic hypothermia and outcome after cardiac arrest. Crit Care Med 2011, 39:2225-2231.

35. Helbok R, Schmidt JM, Kurtz P, Hanafy KA, Fernandez L, Stuart RM, Presciutti M, Ostapkovich ND, Connolly ES, Lee K, et al: Systemic glucose and brain energy metabolism after subarachnoid hemorrhage. Neurocrit Care 2010, 12:317-323.

36. Tseng EE, Brock MV, Lange MS, Troncoso JC, Blue ME, Lowenstein CJ, Johnston MV, Baumgartner WA: Glutamate excitotoxicity mediates neuronal apoptosis after hypothermic circulatory arrest. Ann Thorac Surg 2010, 89:440-445.

37. Berger C, Schabitz WR, Georgiadis D, Steiner T, Aschoff A, Schwab S: Effects of hypothermia on excitatory amino acids and metabolism in stroke patients: a microdialysis study. Stroke 2002, 33:519-524.

38. Zhang S, Zhi D, Lin X, Shang Y, Niu Y: Effect of mild hypothermia on partial pressure of oxygen in brain tissue and brain temperature in patients with severe head injury. Chin J Traumatol 2002, 5:43-45.

39. Maloney-Wilensky E, Le Roux P: The physiology behind direct brain oxygen monitors and practical aspects of their use. Childs Nerv Syst 2010, 26:419-430.

40. Rosenthal G, Hemphill JC, Sorani M, Martin C, Morabito D, Obrist WD, Manley GT: Brain tissue oxygen tension is more indicative of oxygen diffusion than oxygen delivery and metabolism in patients with traumatic brain injury. Crit Care Med 2008, 36:1917-1924. 
41. Gupta AK, Al-Rawi PG, Hutchinson PJ, Kirkpatrick PJ: Effect of hypothermia on brain tissue oxygenation in patients with severe head injury. $\mathrm{Br} J$ Anaesth 2002, 88:188-192.

42. Choi HA, Badjatia N, Mayer SA: Hypothermia for acute brain injury mechanisms and practical aspects. Nat Rev Neurol 2012 in press.

43. Oddo M, Frangos S, Maloney-Wilensky E, Andrew Kofke W, Le Roux PD, Levine JM: Effect of shivering on brain tissue oxygenation during induced normothermia in patients with severe brain injury. Neurocrit Care 2010, 12:10-16.

44. Bisschops LL, Hoedemaekers CW, Simons KS, van der Hoeven JG: Preserved metabolic coupling and cerebrovascular reactivity during mild hypothermia after cardiac arrest. Crit Care Med 2010, 38:1542-1547.

45. Neumann JO, Chambers IR, Citerio G, Enblad P, Gregson BA, Howells T, Mattern J, Nilsson P, Piper I, Ragauskas A, et al: The use of hyperventilation therapy after traumatic brain injury in Europe: an analysis of the BrainIT database. Intensive Care Med 2008, 34:1676-1682.

46. Bratton SL, Chestnut RM, Ghajar J, McConnell Hammond FF, Harris OA, Hartl R, Manley GT, Nemecek A, Newell DW, Rosenthal G, et al: Guidelines for the management of severe traumatic brain injury. XIV. Hyperventilation. J Neurotrauma 2007, 24(Suppl 1):S87-S90.

47. Preisman S, Marks R, Nahtomi-Shick O, Sidi A: Preservation of static and dynamic cerebral autoregulation after mild hypothermic cardiopulmonary bypass. Br J Anaesth 2005, 95:207-211

48. Verhaegen MJ, Todd MM, Hindman BJ, Warner DS: Cerebral autoregulation during moderate hypothermia in rats. Stroke 1993, 24:407-414.

49. Nakamura T, Miyamoto O, Yamagami S, Hayashida Y, Itano T, Nagao S: Influence of rewarming conditions after hypothermia in gerbils with transient forebrain ischemia. J Neurosurg 1999, 91:114-120.

50. Jiang JY, Xu W, Li WP, Gao GY, Bao YH, Liang YM, Luo QZ: Effect of longterm mild hypothermia or short-term mild hypothermia on outcome of patients with severe traumatic brain injury. J Cereb Blood Flow Metab 2006, 26:771-776.

51. Lavinio A, Timofeev I, Nortje J, Outtrim J, Smielewski P, Gupta A, Hutchinson PJ, Matta BF, Pickard JD, Menon D, Czosnyka M: Cerebrovascular reactivity during hypothermia and rewarming. $\mathrm{Br} J$ Anaesth 2007, 99:237-244.

52. Povlishock JT, Wei EP: Posthypothermic rewarming considerations following traumatic brain injury. J Neurotrauma 2009, 26:333-340.

53. Berger C, Xia F, Kohrmann M, Schwab S: Hypothermia in acute stroke slow versus fast rewarming an experimental study in rats. Exp Neurol 2007, 204:131-137.

\section{A28}

Hypothermia and nutrition: at present more questions than answers? Ronny Beer*, Marlene Fischer, Anelia Dietmann, Bettina Pfausler,

Erich Schmutzhard

Neurological Intensive Care Unit, Department of Neurology, Innsbruck

Medical University, Innsbruck, Austria

Critical Care 2012, 16(Suppl 2):A28

Therapeutic hypothermia (TH) has been recently accepted as a powerful medical intervention for providing neuroprotection to patients sustaining cardiac arrest and hypoxic-ischemic encephalopathy. Further, TH has also been explored as a potential treatment strategy for patients with traumatic brain injury and ischemic or hemorrhagic stroke (for review see [1]). With the widespread propagation of $\mathrm{TH}$ as treatment for intracranial hypertension in patients who are refractory to standard interventions [2], induction and maintenance of TH is no longer restricted to the early period (that is, the first 12 to 24 hours) of acute brain injuries but has expanded over several days until rewarming [3]. Therefore, it is evident that, in addition to the disease process itself, TH has a major impact on ICU management such as analgesia and sedation, ventilator therapy, cardiocirculatory support, or artificial nutrition.

Importantly, the significance of nutrition therapy in critically ill patients cannot be overstated [4]. Critical illness typically induces a catabolic stress state proportional to the severity of injury, predisposing patients to serious nutritional deficits coupled with multiple organ dysfunction, delayed recovery and disproportionate mortality [5]. Effective nutrition therapy can play a major role in attenuating the catabolic response and avoiding the harmful effects of prolonged hypermetabolism. International guidelines provide recommendations for timing until initiation of artificial nutrition, administration route, energy targets and type of nutrients in critically ill patients $[4,6]$. Starting early nutrition within 24 to 48 hours, primarily using the enteral route, is a proactive strategy to reduce energy deficit [7]. Importantly, underfeeding and overfeeding must be avoided, which implies monitoring nutritional delivery to timely identify an increasing energy gap or excess administration [8]. Energy requirements are most accurately assessed by measuring resting energy expenditure using indirect calorimetry; however, this method is not widely available [9]. Instead, guideline targets could be applied, with a cautious initial energy requirement of 20 to $25 \mathrm{kcal} / \mathrm{kg} /$ day increasing thereafter in the recovery phase. Enteral nutrition has the advantage of preventing adverse structural and functional alterations of the gut barrier and of improving mesenteric blood flow as well as enhancing local and systemic immune responsiveness [10]. On the other hand, critically ill patients may often have intolerance to gastric feeding [11]. Prokinetic medications may help restore gastric emptying and promote gastrointestinal motility [12]. Persistent intolerance to enteral nutrition selects patients who will require supplemental parenteral nutrition [6]. Another issue in the context of metabolic consequences of nutrition therapy that deserves attention is glycemic control. Both hyperglycemia and hypoglycemia are of particular concern in critically ill patients [13]. However, to date the goal for blood glucose level and whether intensive or a moderate insulin therapy should be employed in these patients, especially with acute brain injuries, is still uncertain [14]. Implementation of a protocol to promote control of serum glucose when providing nutrition therapy is advocated, and a range of 110 to $150 \mathrm{mg} / \mathrm{dl}$ (6.1 to $8.3 \mathrm{mmol} / \mathrm{l})$ may be most appropriate [4].

Whether the aforementioned recommendations on artificial nutrition of the general intensive care patient population can also be applied on equal terms to patients undergoing $\mathrm{TH}$ is still a matter of debate. Studies addressing the various issues of nutrition therapy in $\mathrm{TH}$ are scarce, as evidenced by a contemporary PubMed search including bibliographies of published reviews. So far, data are available on the course of energy expenditure in patients treated with TH suffering from ischemic or hemorrhagic stroke $[15,16]$ and severe traumatic brain injury [2]. In sedated, ventilated patients receiving muscle relaxants a significant decrease in resting energy expenditure ranging at approximately 75 to $85 \%$ of baseline values during $\mathrm{TH}$ could be demonstrated. Downregulation of cerebral and overall metabolism as well as muscle relaxation has been discussed as major factors of the reduction in energy expenditure [15]. In contrast, shivering, an anticipated consequence and potentially major adverse effect of $\mathrm{TH}$, has been shown to be strongly associated with graded increases in systemic metabolism [16]. This clearly indicates the need for an individualized determination of the optimal amount of energy to be delivered during $\mathrm{TH}$. Further, one has to bear in mind that other (patho)physiological changes might occur in patients treated with $\mathrm{TH}$. During the maintenance period of $\mathrm{TH}$, electrolyte replacement is often needed because of a decrease in serum levels of potassium, magnesium and phosphate. With rewarming, these electrolytes are released from intracellular stores and move to extracellular spaces. Therefore, care must be taken to avoid rebound hyperkalemia [17]. In addition, insulin resistance, which can lead to hyperglycemia, may occur during $\mathrm{TH}$. Again, in the rewarming period insulin sensitivity may increase rapidly, resulting in sometimes marked hypoglycemia if the insulin dose is not adjusted appropriately [17].

In conclusion, many questions regarding the nutrition therapy of patients treated with TH remain to be answered. Further studies that focus on the optimal amount of caloric intake, timing, preferred route of administration and monitoring of nutrition delivery during targeted temperature management are urgently needed.

Competing interests: RB received financial support as speaker's fees from Baxter Austria and Fresenius-Kabi Austria.

\section{References}

1. Choi HA, Badjatia N, Mayer SA: Hypothermia for acute brain injurymechanisms and practical aspects. Nat Rev Neurol 2012 in press, doi:10.1038/nrneurol.2012.21.

2. Tokutomi T, Miyagi T, Takeuchi $Y$, et al: Effect of $35^{\circ} \mathrm{C}$ hypothermia on intracranial pressure and clinical outcome in patients with severe traumatic brain injury. J Trauma 2009, 66:166-173.

3. Kollmar R, Juettler $\mathrm{E}$, Huttner $\mathrm{HB}$, et al: Cooling in intracerebral hemorrhage ( $\mathrm{CINCH})$ trial: protocol of a randomized German-Austrian clinical trial. Int J Stroke 2012, 7:168-172. 
4. McClave SA, Martindale RG, Vanek WW, et al: Guidelines for the provision and assessment of nutrition support therapy in the adult critically ill patient: Society of Critical Care Medicine (SCCM) and American Society for Parenteral and Enteral Nutrition (A.S.P.E.N.). JPEN J Parenter Enteral Nutr 2009, 33:277-316.

5. Alberda C, Gramlich L, Jones N, et al: The relationship between nutritional intake and clinical outcomes in critically ill patients: results of an international multicenter observational study. Intensive Care Med 2009, 35:1728-1737.

6. Singer $P$, Berger MM, Van den Berghe $G$, et al: ESPEN guidelines on parenteral nutrition: intensive care. Clin Nutr 2009, 28:387-400.

7. Berger $M M$, Pichard $C$ : Best timing for energy provision during critical illness. Crit Care 2012, 16:215.

8. Stapleton RD, Jones N, Heyland DK: Feeding critically ill patients: what is the optimal amount of energy? Crit Care Med 2007, 35:S535-S540.

9. Singer $P, A n b a r ~ R$, Cohen J, et al: The tight calorie control study (TICACOS): a prospective, randomized, controlled pilot study of nutritional support in critically ill patients. Intensive Care Med 2011, 37:601-609.

10. McClave SA, Heyland DK: The physiologic response and associated clinical benefits from provision of early enteral nutrition. Nutr Clin Pract 2009, 24:305-315.

11. Nguyen NQ, Chapman MJ, Fraser RJ, et al: The effects of sedation on gastric emptying and intra-gastric meal distribution in critical illness. Intensive Care Med 2008, 34:454-460.
12. Dickerson RN, Mitchell JN, Morgan LM, et al: Disparate response to metoclopramide therapy for gastric feeding intolerance in trauma patients with and without traumatic brain injury. JPEN J Parenter Enteral Nutr 2009, 33:646-655.

13. NICE-SUGAR Study Investigators, Finfer S, Chittock DR, et al: Intensive versus conventional glucose control in critically ill patients. N Engl J Med 2009, 360:1283-1297.

14. Oddo M, Schmidt JM, Carrera E, et al: Impact of tight glycemic control on cerebral glucose metabolism after severe brain injury: a microdialysis study. Crit Care Med 2008, 36:3233-3238.

15. Bardutzky J, Georgiadis D, Kollmar R, Schwab S: Energy expenditure in ischemic stroke patients treated with moderate hypothermia. Intensive Care Med 2004, 30:151-154

16. Badjatia N, Strongilis E, Gordon E, et al: Metabolic impact of shivering during therapeutic temperature modulation: the Bedside Shivering Assessment Scale. Stroke 2008, 39:3242-3247.

17. Polderman KH: Mechanisms of action, physiological effects, and complications of hypothermia. Crit Care Med 2009, 37:S186-S202.

Cite abstracts in this supplement using the relevant abstract number, e.g.: Beer et al:: Hypothermia and nutrition: at present more questions than answers?. Critical Care 2012, 16(Suppl 2):A28 